Supporting Information for:

\title{
Double Bonds Are Key to Fast Unimolecular Reactivity in First- Generation Monoterpene Hydroxy Peroxy Radicals
}

Kristian H. Møller*, Rasmus V. Otkjær, Jing Chen, and Henrik G. Kjaergaard*

Department of Chemistry, University of Copenhagen, Universitetsparken 5, DK-2100 Copenhagen Ø, Denmark

(K.H.M.)

E-mail:khm@chem.ku.dk

(H.G.K.)

E-mail: hgk@chem.ku.dk

Phone: +45-35320334. Fax: $+45-35320322$ 


\section{Contents}

S1 Ratios of Rate Coefficients Calculated at Different Levels of Theory .............................................

S2 Rate Coefficients of 1,5-OH H-shifts Calculated Using wB97X-D and M06-2X............................4

S3 Monoterpene Hydroxy Peroxy Radical Rate Coefficients and Related Data .....................................5

S3.1 $\alpha$-Pinene

S3.2 $\beta$-Pinene

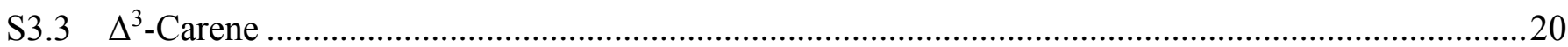

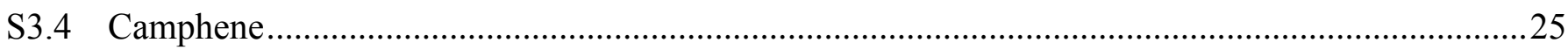

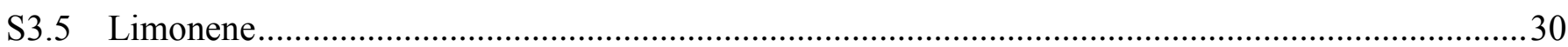

S3.6 Terpinolene

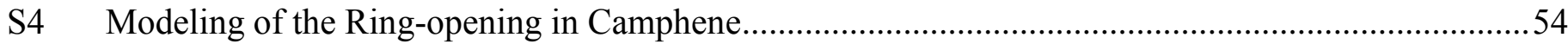

S5 Temperature-Dependence of Reaction Rate Coefficients …............................................................59

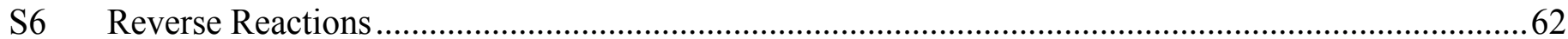

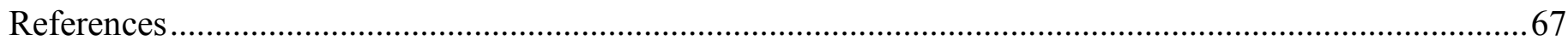




\section{S1 Ratios of Rate Coefficients Calculated at Different Levels of Theory}

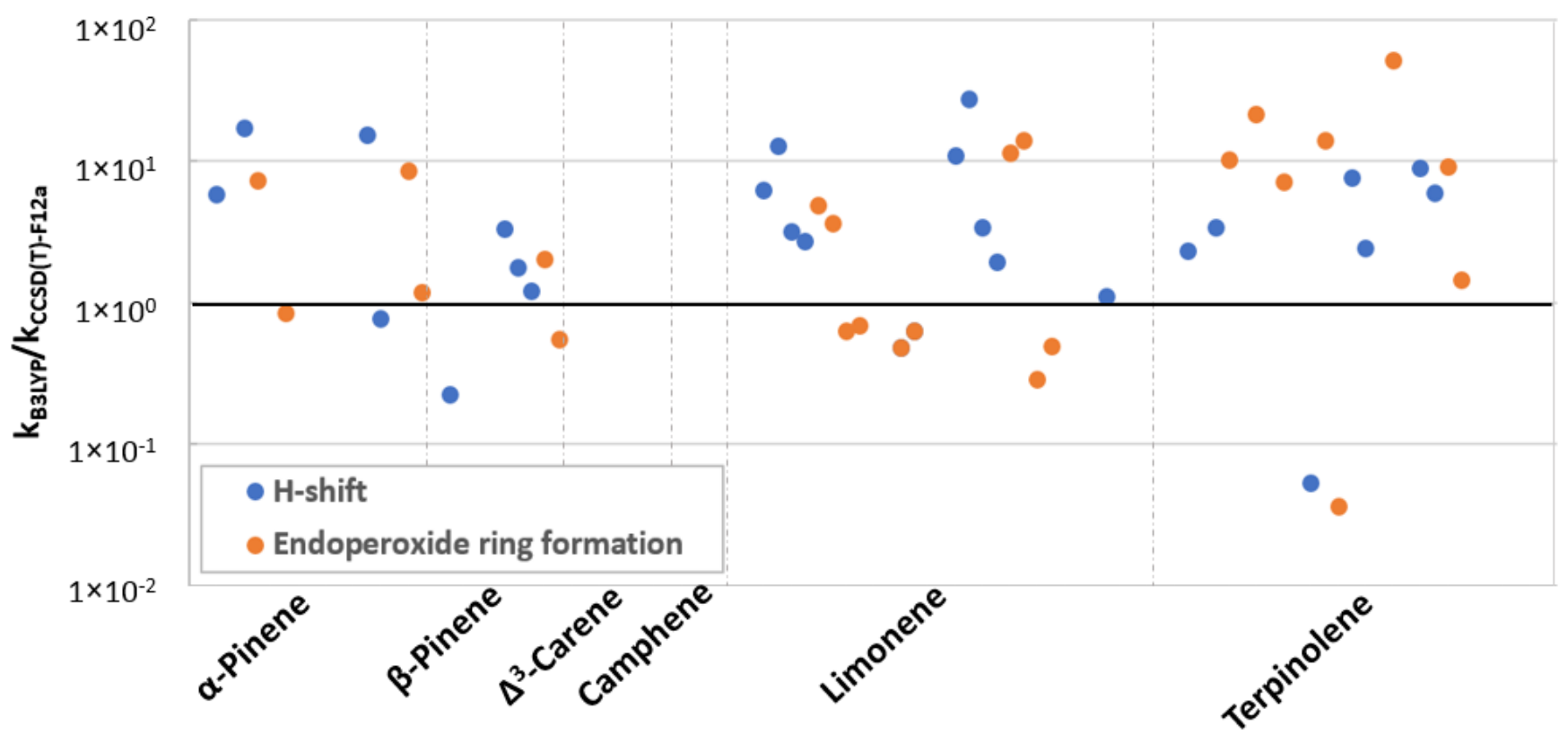

Figure S 1. Ratios of the MC-TST rate coefficients for the unimolecular reactions in the different monoterpene hydroxy peroxy radicals calculated at the B3LYP/6-31+G(d) (kB3LP) and CCSD(T)-F12a/cc-

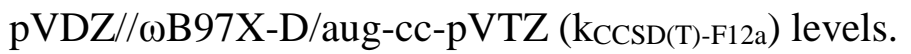

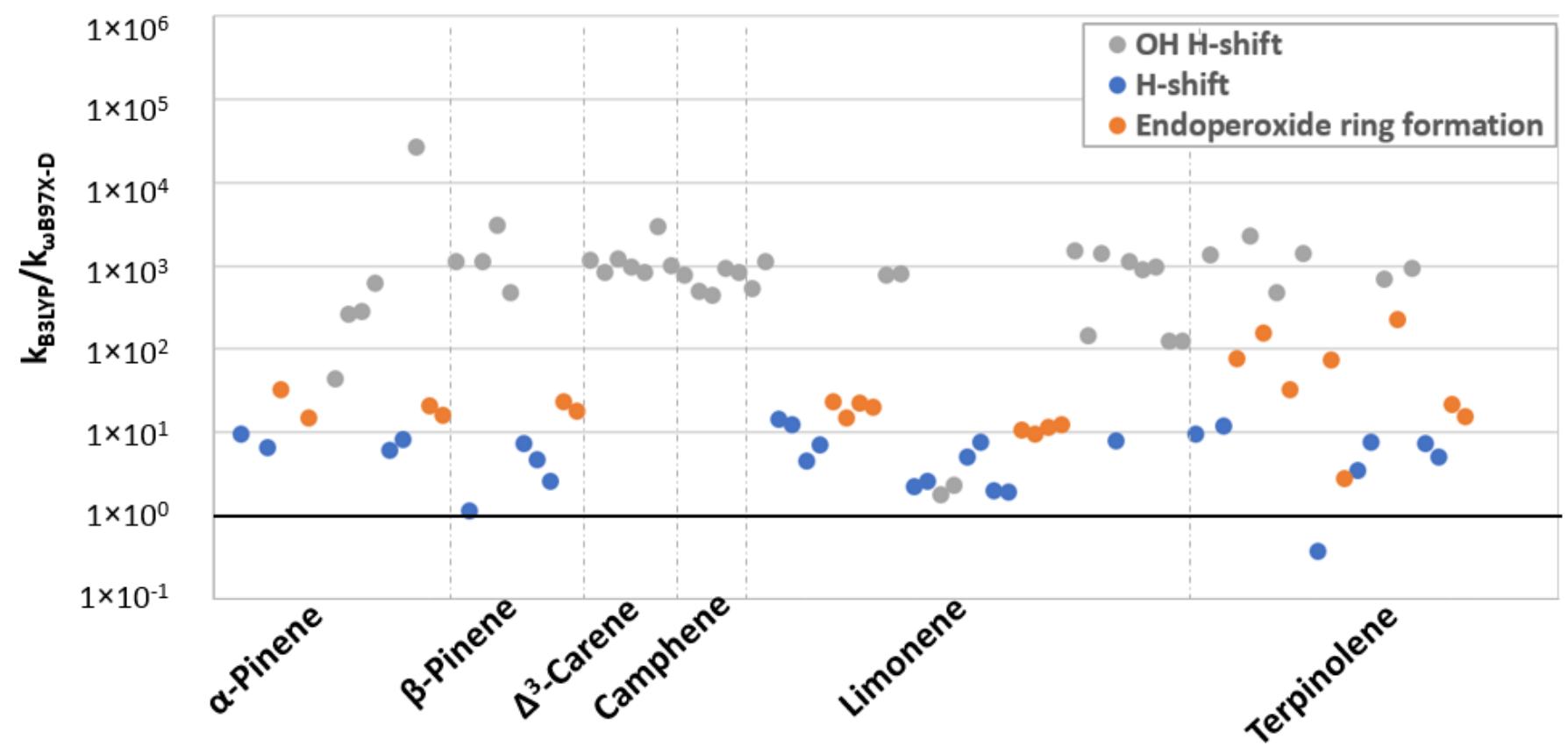

Figure S 2. Ratios of the MC-TST rate coefficients for the unimolecular reactions in the different monoterpene hydroxy peroxy radicals calculated at the B3LYP/6-31+G(d) (kBLYP) and $\omega$ B97X-D/aug-ccpVTZ (k $\mathrm{k}_{\omega 97 \mathrm{X}-\mathrm{D})}$ levels. 


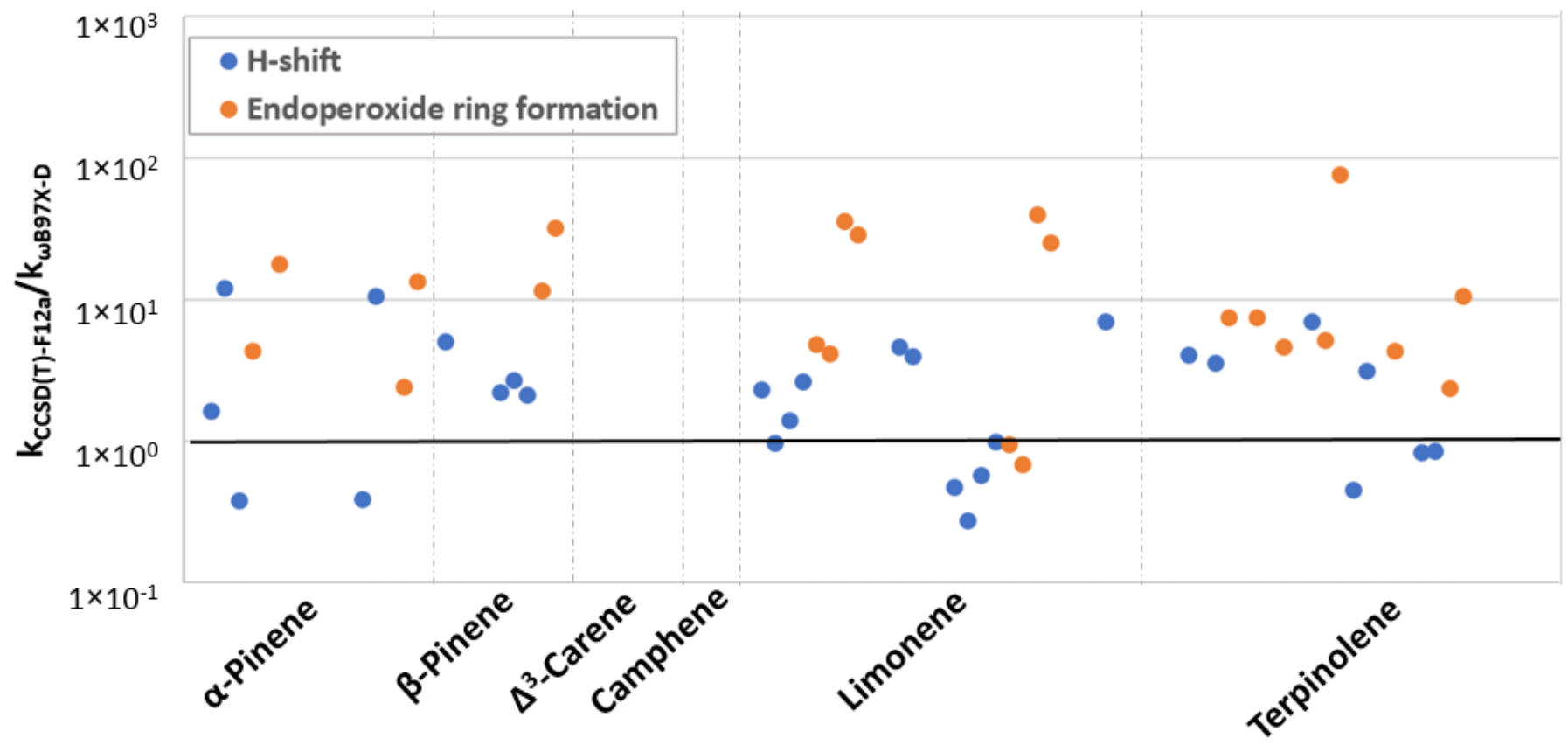

Figure S 3. Ratios of the MC-TST rate coefficients for the unimolecular reactions in the different

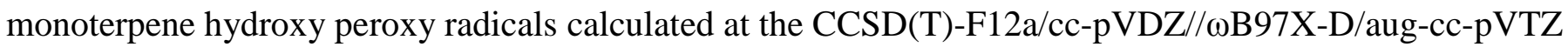
$\left(\mathrm{k}_{\mathrm{CCSD}(\mathrm{T})-\mathrm{F} 12 \mathrm{a})}\right.$ and $\omega \mathrm{B} 97 \mathrm{X}-\mathrm{D} / \mathrm{aug}-\mathrm{cc}-\mathrm{pVTZ}\left(\mathrm{k}_{\omega}\right.$ 97X-D) levels.

\section{S2 Rate Coefficients of 1,5-OH H-shifts Calculated Using $\omega B 97 X-D$ and M06-2X}

Table S 1. MC-TST reaction rate coefficients $\left(\right.$ in s $\left.^{-1}\right)$ calculated using $\omega B$ 97X-D/aug-cc-pVTZ $\left(\mathrm{k}_{\omega \mathrm{B} 97 X-\mathrm{D}}\right)$ and

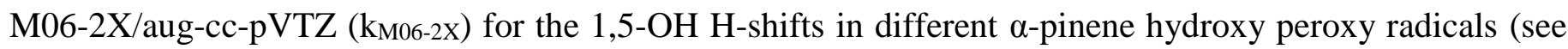
Figure S 5). The conformers are obtained using the approach by Møller et al. ${ }^{1}$ and the two calculations use the same conformers.

\begin{tabular}{|l|l|l|}
\hline Isomer & $\mathrm{k}_{\omega \mathrm{B} 97 \mathrm{X}-\mathrm{D}}$ & $\mathrm{k}_{\mathrm{M} 06-2 \mathrm{X}}$ \\
\hline A2 & $4.7 \times 10^{-2}$ & $2.6 \times 10^{-2}$ \\
\hline A4 & $2.8 \times 10^{-2}$ & $1.5 \times 10^{-2}$ \\
\hline A7 & $1.2 \times 10^{-2}$ & $1.4 \times 10^{-2}$ \\
\hline A9 & $1.2 \times 10^{-1}$ & $9.7 \times 10^{-2}$ \\
\hline A10 & $1.9 \times 10^{-9}$ & $2.4 \times 10^{-9}$ \\
\hline
\end{tabular}




\section{S3 Monoterpene Hydroxy Peroxy Radical Rate Coefficients and Related Data}

\section{S3.1 $\alpha$-Pinene}

The results for $\alpha$-pinene have previously been published by Xu et al. ${ }^{2}$
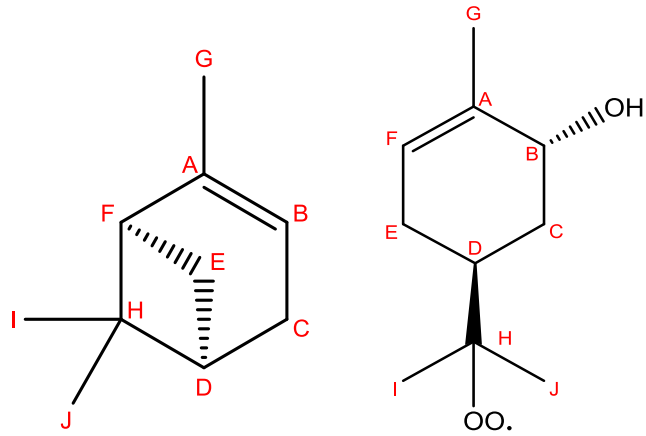

Figure S 4. Structures of $\alpha$-pinene (left) and a ring-opened $\alpha$-pinene peroxy radical (right) with atom labeling of the carbon atoms used to define the unimolecular reactions. 


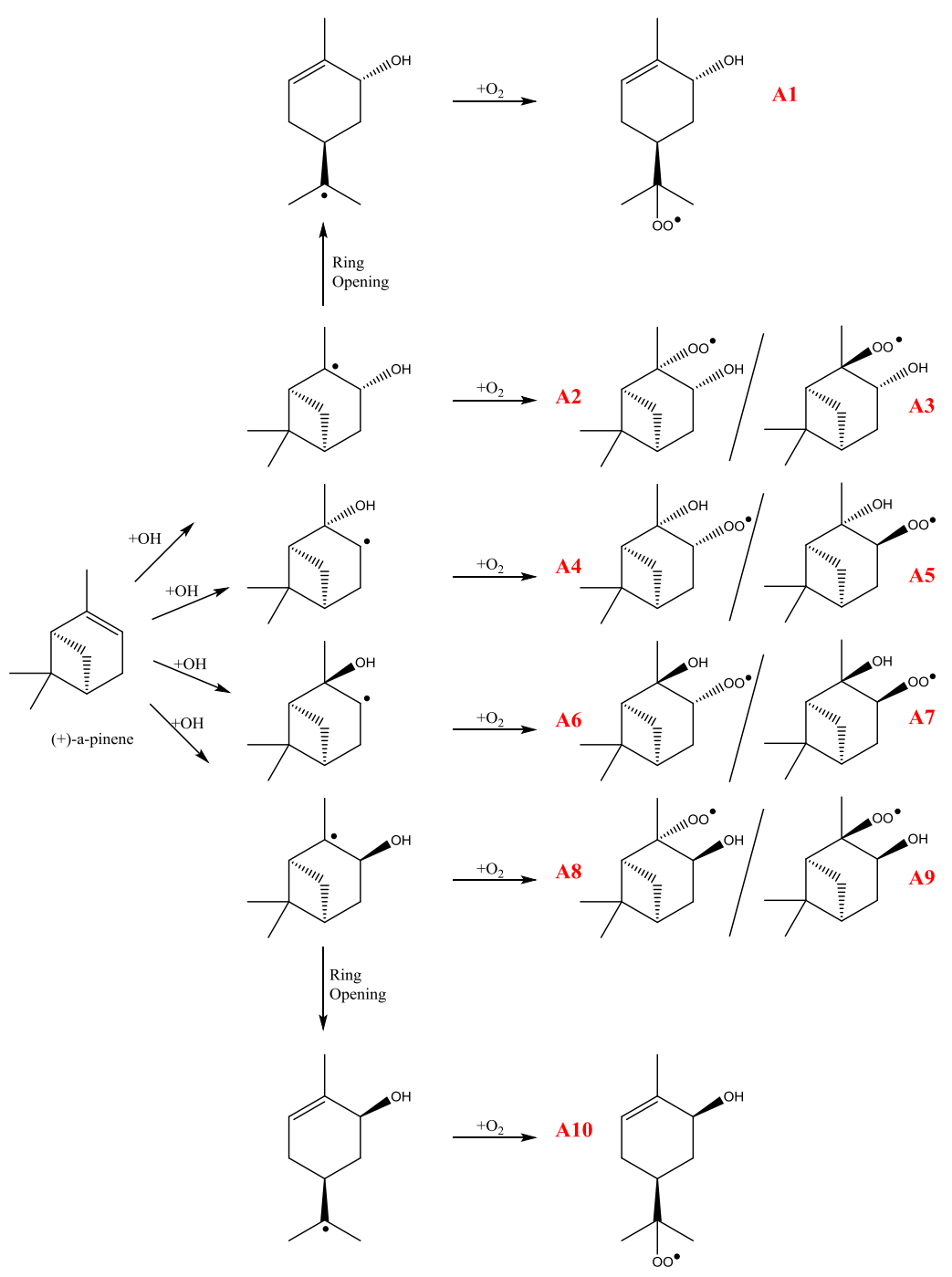

Figure S 5. Formation of the different hydroxy peroxy radicals from $\alpha$-pinene $+\mathrm{OH}+\mathrm{O}_{2}$.

Table S 2. Calculated MC-TST reaction rate coefficients, k, at $298.15 \mathrm{~K}$ for the unimolecular reactions of the hydroxy peroxy radicals formed from $\alpha$-pinene $+\mathrm{OH}+\mathrm{O}_{2}$ (Figure $\mathrm{S} 5$ ). The rate coefficients are calculated using the approach by Møller et al. ${ }^{1}$ The electronic energies of the lowest-energy conformers and IRC endpoints are calculated at the CCSD(T)-F12a/VDZ-F12 level while the remaining parameters are calculated at the $\omega \mathrm{B} 97 \mathrm{X}-\mathrm{D} / \mathrm{aug}-\mathrm{cc}-\mathrm{pVTZ}$ level. Tunneling is based on IRC end-points. The abstraction/addition site refer to the structures in Figure S 4, with "-OH" referring to abstraction of the hydrogen from the hydroxy group on the specified carbon atom. The calculations for A1 were done using Gaussian 09, while the calculations for the other isomers were done using Gaussian 16.

\begin{tabular}{|c|c|c|c|}
\hline Peroxy radical & Reaction type & $\begin{array}{c}\text { Abstraction/addition } \\
\text { site }\end{array}$ & $\mathrm{k}\left(\mathrm{s}^{-1}\right)$ \\
\hline \multirow{3}{*}{$\mathrm{A} 1$} & $1,5 \mathrm{H}$-shift & $\mathrm{E}$ & 1.1 \\
\cline { 2 - 4 } & $1,5 \mathrm{H}$-shift & $\mathrm{C}$ & $6.0 \times 10^{-6}$ \\
\cline { 2 - 4 } & $1,6 \mathrm{H}$-shift & $\mathrm{B}$ & 0.37 \\
\cline { 2 - 4 } & $\begin{array}{c}\text { 6-membered } \\
\text { endoperoxide } \\
\text { formation }\end{array}$ & $\mathrm{F}$ & 0.35 \\
\hline
\end{tabular}




\begin{tabular}{|c|c|c|c|}
\hline & $\begin{array}{l}\text { 7-membered } \\
\text { endoperoxide } \\
\text { formation }\end{array}$ & A & $2.0 \times 10^{-3}$ \\
\hline A2 & 1,5-OH H-shift & $\mathrm{B}-\mathrm{OH}$ & - \\
\hline A4 & 1,5-OH H-shift & $\mathrm{A}-\mathrm{OH}$ & - \\
\hline A7 & 1,5-OH H-shift & $\mathrm{A}-\mathrm{OH}$ & - \\
\hline A9 & 1,5-OH H-shift & $\mathrm{B}-\mathrm{OH}$ & - \\
\hline \multirow{5}{*}{ A10 } & 1,5 H-shift & $E$ & 0.16 \\
\hline & $1,5 \mathrm{H}$-shift & $\mathrm{C}$ & $8.8 \times 10^{-6}$ \\
\hline & 1,7-OH H-shift & $\mathrm{B}-\mathrm{OH}$ & - \\
\hline & $\begin{array}{l}\text { 6-membered } \\
\text { endoperoxide } \\
\text { formation }\end{array}$ & $\mathrm{F}$ & 2.3 \\
\hline & $\begin{array}{l}\text { 7-membered } \\
\text { endoperoxide } \\
\text { formation }\end{array}$ & A & $2.6 \times 10^{-2}$ \\
\hline
\end{tabular}


Table S 3. Calculated MC-TST reaction rate coefficients, $\mathrm{k}$, at $298.15 \mathrm{~K}$ for the unimolecular reactions of the hydroxy peroxy radicals formed from $\alpha$-pinene $+\mathrm{OH}+\mathrm{O}_{2}$ (Figure $\mathrm{S} 5$ ). All values are calculated at the $\omega B 97 X-D / a u g-c c-p V T Z$ level of theory with tunneling based on IRC end-points. The abstraction/addition site refer to the structures in Figure S 4, with "-OH" referring to abstraction of the hydrogen from the hydroxy group on the specified carbon atom. The calculations for A1 were done using Gaussian 09, while the calculations for the other isomers were done using Gaussian 16.

\begin{tabular}{|c|c|c|c|}
\hline Peroxy radical & Reaction type & $\begin{array}{c}\text { Abstraction/addition } \\
\text { site }\end{array}$ & $\mathrm{k}\left(\mathrm{s}^{-1}\right)$ \\
\hline \multirow{5}{*}{ A1 } & $1,5 \mathrm{H}$-shift & $\mathrm{E}$ & $0.66^{\mathrm{a}}$ \\
\hline & 1,5 H-shift & $\mathrm{C}$ & $4.9 \times 10^{-7}$ \\
\hline & $1,6 \mathrm{H}$-shift & $\mathrm{B}$ & $0.96^{\mathrm{a}}$ \\
\hline & $\begin{array}{c}\text { 6-membered } \\
\text { endoperoxide } \\
\text { formation }\end{array}$ & $\mathrm{F}$ & $7.9 \times 10^{-2} \mathrm{a}$ \\
\hline & $\begin{array}{c}\text { 7-membered } \\
\text { endoperoxide } \\
\text { formation }\end{array}$ & A & $1.1 \times 10^{-4}$ \\
\hline \multirow{2}{*}{ A2 } & 1,5-OH H-shift & $\mathrm{B}-\mathrm{OH}$ & $4.7 \times 10^{-2}$ \\
\hline & 1,5 H-shift & $E$ & $1.4 \times 10^{-3}$ \\
\hline A4 & 1,5-OH H-shift & $\mathrm{A}-\mathrm{OH}$ & $2.8 \times 10^{-2}$ \\
\hline A7 & 1,5-OH H-shift & $\mathrm{A}-\mathrm{OH}$ & $1.2 \times 10^{-2}$ \\
\hline A9 & 1,5-OH H-shift & $\mathrm{B}-\mathrm{OH}$ & 0.12 \\
\hline \multirow{5}{*}{ A10 } & $1,5 \mathrm{H}$-shift & $\mathrm{E}$ & 0.41 \\
\hline & 1,5 H-shift & $\mathrm{C}$ & $8.3 \times 10^{-7}$ \\
\hline & 1,7-OH H-shift & $\mathrm{B}-\mathrm{OH}$ & $1.9 \times 10^{-9}$ \\
\hline & $\begin{array}{c}\text { 6-membered } \\
\text { endoperoxide } \\
\text { formation }\end{array}$ & $\mathrm{F}$ & 0.95 \\
\hline & $\begin{array}{l}\text { 7-membered } \\
\text { endoperoxide } \\
\text { formation }\end{array}$ & A & $1.9 \times 10^{-3}$ \\
\hline
\end{tabular}

${ }^{\text {a }}$ Also reported in Berndt et al. ${ }^{3}$ 
Table S 4. Calculated reaction rate coefficients, $\mathrm{k}$, at $298.15 \mathrm{~K}$ for the unimolecular reactions of the hydroxy peroxy radicals formed from $\alpha$-pinene $+\mathrm{OH}+\mathrm{O}_{2}$ (Figure $\mathrm{S} 5$ ). All values are calculated at the B3LYP/6$31+\mathrm{G}(\mathrm{d})$ level of theory with the symmetrical Eckart tunneling approach assuming thermoneutral reactions. The abstraction/addition site refer to the structures in Figure S 4, with "-OH" referring to abstraction of the hydrogen from the hydroxy group on the specified carbon atom. The reactions highlighted in bold are the ones also treated at a higher level of theory. The calculations for A1 were done using Gaussian 09, while the calculations for the other isomers were done using Gaussian 16.

\begin{tabular}{|c|c|c|c|}
\hline Peroxy radical & Reaction type & $\begin{array}{l}\text { Abstraction/addition } \\
\text { site }\end{array}$ & $\mathrm{k}\left(\mathrm{s}^{-1}\right)$ \\
\hline \multirow{7}{*}{ A1 } & 1,5 H-shift & $\mathbf{E}$ & 6.5 \\
\hline & 1,5 H-shift & $\mathbf{C}$ & $6.7 \times 10^{-6}$ \\
\hline & 1,6 H-shift & B & 6.4 \\
\hline & 1,6 H-shift & $\mathrm{F}$ & $1.8 \times 10^{-5}$ \\
\hline & 1,7-OH H-shift & $\mathrm{B}-\mathrm{OH}$ & $1.6 \times 10^{-3}$ \\
\hline & $\begin{array}{l}\text { 6-membered } \\
\text { endoperoxide } \\
\text { formation }\end{array}$ & $\mathbf{F}$ & 2.6 \\
\hline & $\begin{array}{l}\text { 7-membered } \\
\text { endoperoxide } \\
\text { formation }\end{array}$ & $\mathbf{A}$ & $1.7 \times 10^{-3}$ \\
\hline \multirow{6}{*}{$\mathrm{A} 2$} & 1,4 H-shift & $\mathrm{F}$ & $2.9 \times 10^{-10}$ \\
\hline & 1,5 H-shift & $\mathrm{C}$ & $8.5 \times 10^{-15}$ \\
\hline & 1,4 H-shift & $\mathrm{B}$ & $5.8 \times 10^{-20}$ \\
\hline & 1,5-OH H-shift & B-OH & 21 \\
\hline & 1,5 H-shift & $\mathrm{E}$ & $3.6 \times 10^{-3}$ \\
\hline & 1,4 H-shift & $\mathrm{G}$ & $8.2 \times 10^{-9}$ \\
\hline \multirow{6}{*}{ A3 } & 1,4 H-shift & $\mathrm{F}$ & $6.0 \times 10^{-11}$ \\
\hline & 1,5 H-shift & $\mathrm{C}$ & $8.0 \times 10^{-19}$ \\
\hline & 1,4 H-shift & $\mathrm{B}$ & $5.1 \times 10^{-4}$ \\
\hline & 1,5-OH H-shift & $\mathrm{B}-\mathrm{OH}$ & $2.2 \times 10^{-7}$ \\
\hline & 1,6 H-shift & $\mathrm{I}$ & $1.1 \times 10^{-7}$ \\
\hline & 1,4 H-shift & $\mathrm{G}$ & $1.3 \times 10^{-9}$ \\
\hline \multirow{3}{*}{ A4 } & 1,4 H-shift & $\mathrm{C}$ & $2.8 \times 10^{-7}$ \\
\hline & 1,5-OH H-shift & A-OH & 7.4 \\
\hline & 1,6 H-shift & $\mathrm{E}$ & $1.2 \times 10^{-7}$ \\
\hline \multirow{4}{*}{ A5 } & 1,4 H-shift & $\mathrm{C}$ & $4.3 \times 10^{-8}$ \\
\hline & 1,5 H-shift & $\mathrm{G}$ & $5.0 \times 10^{-5}$ \\
\hline & 1,5-OH H-shift & $\mathrm{A}-\mathrm{OH}$ & $1.9 \times 10^{-5}$ \\
\hline & 1,7 H-shift & I & $5.0 \times 10^{-8}$ \\
\hline \multirow{4}{*}{ A6 } & 1,4 H-shift & $\mathrm{C}$ & $3.1 \times 10^{-7}$ \\
\hline & 1,5 H-shift & $\mathrm{G}$ & $4.7 \times 10^{-5}$ \\
\hline & 1,5-OH H-shift & $\mathrm{A}-\mathrm{OH}$ & $5.3 \times 10^{-6}$ \\
\hline & 1,6 H-shift & $\mathrm{E}$ & $7.1 \times 10^{-8}$ \\
\hline
\end{tabular}




\begin{tabular}{|c|c|c|c|}
\hline \multirow{4}{*}{ A7 } & 1,4 H-shift & $\mathrm{C}$ & $1.4 \times 10^{-8}$ \\
\hline & $1,5 \mathrm{H}$-shift & G & $2.9 \times 10^{-8}$ \\
\hline & 1,5-OH H-shift & A-OH & 3.5 \\
\hline & 1,7 H-shift & I & $2.7 \times 10^{-6}$ \\
\hline \multirow{6}{*}{ A8 } & 1,4 H-shift & $\mathrm{F}$ & $1.3 \times 10^{-10}$ \\
\hline & 1,5 H-shift & $\mathrm{C}$ & $1.2 \times 10^{-19}$ \\
\hline & 1,4 H-shift & $\mathrm{B}$ & $1.9 \times 10^{-3}$ \\
\hline & 1,5-OH H-shift & $\mathrm{B}-\mathrm{OH}$ & $6.5 \times 10^{-7}$ \\
\hline & $1,5 \mathrm{H}$-shift & $\mathrm{E}$ & $1.3 \times 10^{-10}$ \\
\hline & 1,4 H-shift & G & $2.5 \times 10^{-9}$ \\
\hline \multirow{6}{*}{ A9 } & 1,4 H-shift & $\mathrm{F}$ & $1.2 \times 10^{-11}$ \\
\hline & 1,5 H-shift & $\mathrm{C}$ & $6.4 \times 10^{-16}$ \\
\hline & 1,4 H-shift & $\mathrm{B}$ & $2.8 \times 10^{-23}$ \\
\hline & 1,5-OH H-shift & B-OH & 75 \\
\hline & $1,6 \mathrm{H}$-shift & I & $1.6 \times 10^{-4}$ \\
\hline & 1,4 H-shift & $\mathrm{G}$ & $8.9 \times 10^{-10}$ \\
\hline \multirow{5}{*}{ A10 } & 1,5 H-shift & $\mathbf{E}$ & 2.5 \\
\hline & 1,5 H-shift & $\mathrm{C}$ & $6.9 \times 10^{-6}$ \\
\hline & 1,7-OH H-shift & B-OH & $5.2 \times 10^{-5}$ \\
\hline & $\begin{array}{l}\text { 6-membered } \\
\text { endoperoxide } \\
\text { formation }\end{array}$ & $\mathbf{F}$ & 20 \\
\hline & $\begin{array}{c}\text { 7-membered } \\
\text { endoperoxide } \\
\text { formation }\end{array}$ & $\mathbf{A}$ & $3.1 \times 10^{-2}$ \\
\hline
\end{tabular}


Table S 5. CCSD(T)-F12a/VDZ-F12 (abbreviated F12) and $\omega$ B97X-D/aug-cc-pVTZ barrier heights ( $\mathrm{E}_{0}$, in $\mathrm{kcal} / \mathrm{mol})$, summed partition function ratios $\left(\mathrm{Q}_{\mathrm{TS}} / \mathrm{Q}_{\mathrm{R}}\right)$ and Eckart tunneling coefficients $(\kappa)$ used for calculating the corresponding MC-TST rate coefficients in the preceding tables for $\alpha$-pinene. The calculations for A1 were done using Gaussian 09, while the calculations for the other isomers were done using Gaussian 16.

\begin{tabular}{|c|c|c|c|c|c|c|c|}
\hline $\begin{array}{l}\text { Peroxy } \\
\text { radical }\end{array}$ & $\begin{array}{l}\text { Reaction } \\
\text { type }\end{array}$ & $\begin{array}{l}\text { Abstraction/ } \\
\text { addition site }\end{array}$ & $\begin{array}{c}\mathrm{E}_{0} \\
(\mathrm{~F} 12)\end{array}$ & $\begin{array}{c}\kappa \\
(\mathrm{F} 12)\end{array}$ & $\begin{array}{c}E_{0} \\
(\omega B 97 X-D)\end{array}$ & $\mathrm{Q}_{\mathrm{TS}} / \mathrm{Q}_{\mathrm{R}}$ & $\begin{array}{c}\kappa \\
(\omega \mathrm{B} 97 \mathrm{X}-\mathrm{D})\end{array}$ \\
\hline \multirow{5}{*}{ A1 } & $1,5 \mathrm{H}$-shift & $\mathrm{E}$ & 19.5 & 626.3 & 19.8 & $6.12 \times 10^{-2}$ & 535.6 \\
\hline & 1,5 H-shift & $\mathrm{C}$ & 25.4 & 29.8 & 26.7 & $1.46 \times 10^{-1}$ & 20.6 \\
\hline & 1,6 H-shift & $\mathrm{B}$ & 18.4 & 58.9 & 17.7 & $2.97 \times 10^{-2}$ & 51.0 \\
\hline & $\begin{array}{c}\text { 6-membered } \\
\text { endoperoxide } \\
\text { formation }\end{array}$ & $\mathrm{F}$ & 16.3 & 1.5 & 17.2 & $3.47 \times 10^{-2}$ & 1.5 \\
\hline & $\begin{array}{c}\text { 7-membered } \\
\text { endoperoxide } \\
\text { formation }\end{array}$ & A & 19.3 & 1.4 & 21.0 & $3.02 \times 10^{-2}$ & 1.4 \\
\hline \multirow{2}{*}{$\mathrm{A} 2$} & 1,5-OH H-shift & $\mathrm{B}-\mathrm{OH}$ & - & - & 18.3 & $1.88 \times 10^{-1}$ & 1.0 \\
\hline & 1,5 H-shift & $\mathrm{E}$ & - & - & 21.1 & $1.36 \times 10^{-1}$ & 5.0 \\
\hline A4 & 1,5-OH H-shift & $\mathrm{A}-\mathrm{OH}$ & - & - & 19.2 & $5.10 \times 10^{-1}$ & 1.0 \\
\hline A7 & 1,5-OH H-shift & $\mathrm{A}-\mathrm{OH}$ & - & - & 19.6 & $2.63 \times 10^{-1}$ & 1.8 \\
\hline A9 & 1,5-OH H-shift & $\mathrm{B}-\mathrm{OH}$ & - & - & 17.8 & $1.63 \times 10^{-1}$ & 1.28 \\
\hline \multirow{5}{*}{ A10 } & 1,5 H-shift & $\mathrm{E}$ & 18.9 & 139.9 & 18.3 & $1.36 \times 10^{-2}$ & 122.5 \\
\hline & 1,5 H-shift & $\mathrm{C}$ & 25.0 & 43.9 & 26.1 & $6.48 \times 10^{-2}$ & 26.4 \\
\hline & 1,7-OH H-shift & $\mathrm{B}-\mathrm{OH}$ & 35.1 & 6.5 & 27.8 & $2.03 \times 10^{-2}$ & 3.7 \\
\hline & $\begin{array}{c}\text { 6-membered } \\
\text { endoperoxide } \\
\text { formation }\end{array}$ & $\mathrm{F}$ & 14.2 & 1.4 & 14.7 & $6.91 \times 10^{-3}$ & 1.4 \\
\hline & $\begin{array}{l}\text { 7-membered } \\
\text { endoperoxide } \\
\text { formation }\end{array}$ & A & 16.8 & 1.3 & 18.4 & $6.91 \times 10^{-3}$ & 1.3 \\
\hline
\end{tabular}


Table S 6. B3LYP/6-31+G(d) barrier heights ( $\mathrm{E}_{0}$, in $\left.\mathrm{kcal} / \mathrm{mol}\right)$, summed partition function ratios $\left(\mathrm{Q}_{\mathrm{TS}} / \mathrm{Q}_{\mathrm{R}}\right)$ and Eckart tunneling coefficients $(\kappa)$ used for calculating the corresponding MC-TST rate coefficients in the preceding tables for $\alpha$-pinene. The calculations for A1 were done using Gaussian 09, while the calculations for the other isomers were done using Gaussian 16.

\begin{tabular}{|c|c|c|c|c|c|}
\hline $\begin{array}{l}\text { Peroxy } \\
\text { radical }\end{array}$ & Reaction type & $\begin{array}{c}\text { Abstraction/addition } \\
\text { site }\end{array}$ & $\mathrm{E}_{0}$ & $\mathrm{Q}_{\mathrm{TS}} / \mathrm{Q}_{\mathrm{R}}$ & $\kappa$ \\
\hline \multirow{7}{*}{ A1 } & 1,5 H-shift & $\mathbf{E}$ & 16.1 & 0.06896 & 91.1 \\
\hline & 1,5 H-shift & $\mathbf{C}$ & 24.4 & 0.11130 & 196.8 \\
\hline & 1,6 H-shift & B & 14.5 & 0.01997 & 18.5 \\
\hline & 1,6 H-shift & $\mathrm{F}$ & 44.1 & 0.03076 & 41164.0 \\
\hline & 1,7-OH H-shift & $\mathrm{B}-\mathrm{OH}$ & 39.1 & 0.00928 & 804.0 \\
\hline & $\begin{array}{l}\text { 6-membered } \\
\text { endoperoxide } \\
\text { formation } \\
\end{array}$ & $\mathbf{F}$ & 15.0 & 0.03224 & 1.3 \\
\hline & $\begin{array}{l}\text { 7-membered } \\
\text { endoperoxide } \\
\text { formation } \\
\end{array}$ & $\mathbf{A}$ & 19.3 & 0.02914 & 1.2 \\
\hline \multirow{6}{*}{$\mathrm{A} 2$} & 1,4 H-shift & $\mathrm{F}$ & 35.8 & 0.22045 & 38817.0 \\
\hline & $1,5 \mathrm{H}$-shift & $\mathrm{C}$ & 39.2 & 0.13779 & 556.5 \\
\hline & 1,4 H-shift & $\mathrm{B}$ & 48.8 & 0.08924 & 59571.0 \\
\hline & 1,5-OH H-shift & B-OH & 15.3 & 0.16076 & 3.8 \\
\hline & $1,5 \mathrm{H}$-shift & $\mathrm{E}$ & 21.1 & 0.10975 & 16.3 \\
\hline & 1,4 H-shift & $\mathrm{G}$ & 34.8 & 0.39520 & 112050.0 \\
\hline \multirow{6}{*}{ A3 } & 1,4 H-shift & $\mathrm{F}$ & 37.4 & 0.48588 & 54793.0 \\
\hline & $1,5 \mathrm{H}$-shift & $\mathrm{C}$ & 49.4 & 0.26892 & 801650.0 \\
\hline & 1,4 H-shift & $\mathrm{B}$ & 25.5 & 0.30173 & 1283.9 \\
\hline & 1,5-OH H-shift & $\mathrm{B}-\mathrm{OH}$ & 28.8 & 0.15374 & 270.6 \\
\hline & 1,6 H-shift & $\mathrm{I}$ & 27.7 & 0.17796 & 18.4 \\
\hline & 1,4 H-shift & $\mathrm{G}$ & 36.2 & 0.51907 & 133550.0 \\
\hline \multirow{3}{*}{ A4 } & 1,4 H-shift & $\mathrm{C}$ & 31.4 & 0.39784 & 11516.0 \\
\hline & 1,5-OH H-shift & A-OH & 16.4 & 0.38975 & 3.3 \\
\hline & 1,6 H-shift & $\mathrm{E}$ & 28.8 & 0.15197 & 160.1 \\
\hline \multirow{4}{*}{ A5 } & 1,4 H-shift & $\mathrm{C}$ & 32.8 & 0.20303 & 39044.0 \\
\hline & $1,5 \mathrm{H}$-shift & $\mathrm{G}$ & 24.6 & 0.10534 & 90.2 \\
\hline & 1,5-OH H-shift & $\mathrm{A}-\mathrm{OH}$ & 25.4 & 0.07336 & 172.7 \\
\hline & 1,7 H-shift & I & 28.0 & 0.11602 & 22.8 \\
\hline \multirow{4}{*}{ A6 } & 1,4 H-shift & $\mathrm{C}$ & 31.4 & 0.22498 & 24555.0 \\
\hline & $1,5 \mathrm{H}$-shift & $\mathrm{G}$ & 24.7 & 0.15396 & 67.4 \\
\hline & 1,5-OH H-shift & $\mathrm{A}-\mathrm{OH}$ & 26.2 & 0.09435 & 140.8 \\
\hline & 1,6 H-shift & $E$ & 29.4 & 0.14036 & 284.6 \\
\hline \multirow{3}{*}{ A7 } & 1,4 H-shift & $\mathrm{C}$ & 33.7 & 0.33505 & 36008.0 \\
\hline & $1,5 \mathrm{H}$-shift & $\mathrm{G}$ & 30.2 & 0.11430 & 575.4 \\
\hline & 1,5-OH H-shift & A-OH & 16.9 & 0.29890 & 4.5 \\
\hline
\end{tabular}




\begin{tabular}{|c|c|c|c|c|c|}
\hline & 1,7 H-shift & $\mathrm{I}$ & 26.2 & 0.09342 & 73.1 \\
\hline \multirow{6}{*}{ A8 } & 1,4 H-shift & $\mathrm{F}$ & 36.9 & 0.41497 & 52549.0 \\
\hline & 1,5 H-shift & $\mathrm{C}$ & 51.9 & 0.57172 & 3827700.0 \\
\hline & 1,4 H-shift & $\mathrm{B}$ & 24.8 & 0.34957 & 1333.9 \\
\hline & 1,5-OH H-shift & $\mathrm{B}-\mathrm{OH}$ & 27.8 & 0.13781 & 195.5 \\
\hline & $1,5 \mathrm{H}$-shift & $E$ & 26.2 & 0.23819 & 22.0 \\
\hline & 1,4 H-shift & $\mathrm{G}$ & 35.6 & 0.34722 & 152590.0 \\
\hline \multirow{6}{*}{ A9 } & 1,4 H-shift & $\mathrm{F}$ & 38.0 & 0.29944 & 44856.0 \\
\hline & 1,5 H-shift & $\mathrm{C}$ & 42.4 & 0.11632 & 10167.0 \\
\hline & 1,4 H-shift & $\mathrm{B}$ & 53.8 & 0.17489 & 69536.0 \\
\hline & 1,5-OH H-shift & B-OH & 14.8 & 0.19289 & 4.7 \\
\hline & 1,6 H-shift & $\mathrm{I}$ & 23.0 & 0.08920 & 20.1 \\
\hline & 1,4 H-shift & $\mathrm{G}$ & 35.9 & 0.31533 & 92761.0 \\
\hline \multirow{5}{*}{ A10 } & 1,5 H-shift & $\mathbf{E}$ & 17.0 & 0.02407 & 45.4 \\
\hline & 1,5 H-shift & $\mathbf{C}$ & 25.6 & 0.05268 & 118.7 \\
\hline & 1,7-OH H-shift & B-OH & 23.3 & 0.01448 & 66.6 \\
\hline & $\begin{array}{c}\text { 6-membered } \\
\text { endoperoxide } \\
\text { formation }\end{array}$ & $\mathbf{F}$ & 12.8 & 0.00574 & 1.3 \\
\hline & $\begin{array}{c}\text { 7-membered } \\
\text { endoperoxide } \\
\text { formation }\end{array}$ & $\mathbf{A}$ & 16.5 & 0.00499 & 1.2 \\
\hline
\end{tabular}




\section{S3.2 $\beta$-Pinene}

The results for $\beta$-pinene have previously been published by Xu et al. ${ }^{2}$
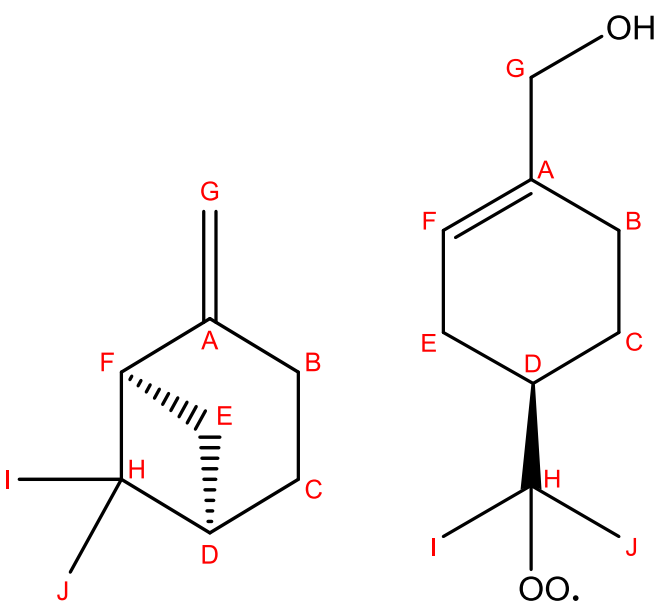

Figure S 6. Structures of $\beta$-pinene (left) and the ring-opened $\beta$-pinene peroxy radical (right) with atom labeling of the carbon atoms used to define the unimolecular reactions.

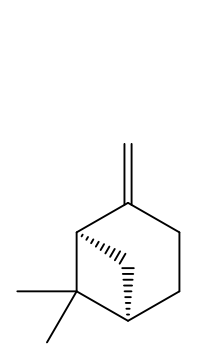

(+)-b-pinene

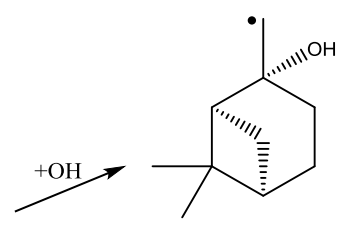<smiles>CC(C)CC(C)C</smiles><smiles>CC1(C)[C@H]2CC[C@@H](CO)[C@H]1CC2</smiles><smiles>CC(C)[C@H]1CC=C(CO)CC1</smiles>
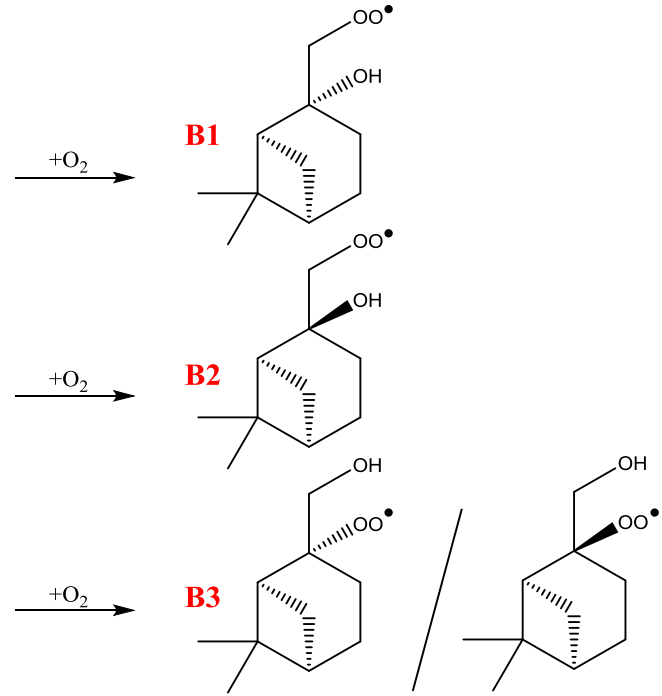

B4

Figure S 7. Formation of the different hydroxy peroxy radicals from $\beta$-pinene $+\mathrm{OH}+\mathrm{O}_{2}$. 
Table S 7. Calculated MC-TST reaction rate coefficients, $\mathrm{k}$, at $298.15 \mathrm{~K}$ for the unimolecular reactions of the hydroxy peroxy radicals formed from $\beta$-pinene $+\mathrm{OH}+\mathrm{O}_{2}$ (Figure S 7). The rate coefficients are calculated using the approach by Møller et al. ${ }^{1}$ The electronic energies of the lowest-energy conformers and IRC endpoints are calculated at the CCSD(T)-F12a/VDZ-F12 level while the remaining parameters are calculated at the $\omega$ B97X-D/aug-cc-pVTZ level. Tunneling is based on IRC end-points. The abstraction/addition site refer to the structures in Figure S 6, with "-OH" referring to abstraction of the hydrogen from the hydroxy group on the specified carbon atom.

\begin{tabular}{|c|c|c|c|}
\hline \multirow{2}{*}{ Peroxy radical } & Reaction type & $\begin{array}{c}\text { Abstraction/addition } \\
\text { site }\end{array}$ & $\mathrm{k}\left(\mathrm{s}^{-1}\right)$ \\
\hline B1 & $1,5-\mathrm{OH}$ H-shift & $\mathrm{A}-\mathrm{OH}$ & - \\
\hline \multirow{2}{*}{ B2 } & 1,5 H-shift & $\mathrm{B}$ & $8.1 \times 10^{-2}$ \\
\cline { 2 - 4 } & $1,5-\mathrm{OH}$ H-shift & $\mathrm{A}-\mathrm{OH}$ & - \\
\hline B3 & $1,5-\mathrm{OH}$ H-shift & $\mathrm{G}-\mathrm{OH}$ & - \\
\hline B4 & $\begin{array}{c}\text { 1,5-OH H-shift } \\
\text { B5 }\end{array}$ & G-OH & $7.3 \times 10^{-6}$ \\
\cline { 2 - 4 } & 1,5 H-shift & $\mathrm{E}$ & 0.28 \\
\cline { 2 - 4 } & $\begin{array}{c}1,6 \text { H-shift } \\
\text { 6-membered } \\
\text { Endoperoxide } \\
\text { formation }\end{array}$ & $\mathrm{C}$ & 4.0 \\
\cline { 2 - 4 } & $\begin{array}{c}\text { 7-membered } \\
\text { Endoperoxide } \\
\text { formation }\end{array}$ & $\mathrm{F}$ & $4.8 \times 10^{-2}$ \\
\hline
\end{tabular}


Table S 8. Calculated MC-TST reaction rate coefficients, $\mathrm{k}$, at $298.15 \mathrm{~K}$ for the unimolecular reactions of the hydroxy peroxy radicals formed from $\beta$-pinene $+\mathrm{OH}+\mathrm{O}_{2}$ (Figure $\mathrm{S} 7$ ). All values are calculated at the $\omega B 97 X-D / a u g-c c-p V T Z$ level of theory with tunneling based on IRC end-points. The abstraction/addition site refer to the structures in Figure S 6, with "-OH" referring to abstraction of the hydrogen from the hydroxy group on the specified carbon atom.

\begin{tabular}{|c|c|c|c|}
\hline Peroxy radical & Reaction type & $\begin{array}{l}\text { Abstraction/addition } \\
\text { site }\end{array}$ & $\mathrm{k}\left(\mathrm{s}^{-1}\right)$ \\
\hline B1 & 1,5-OH H-shift & $\mathrm{A}-\mathrm{OH}$ & $5.3 \times 10^{-4}$ \\
\hline \multirow{3}{*}{ B2 } & 1,5 H-shift & $\mathrm{F}$ & $4.7 \times 10^{-5}$ \\
\hline & $1,5 \mathrm{H}$-shift & $\mathrm{B}$ & $1.6 \times 10^{-2}$ \\
\hline & 1,5-OH H-shift & $\mathrm{A}-\mathrm{OH}$ & $1.9 \times 10^{-2}$ \\
\hline B3 & 1,5-OH H-shift & G-OH & $2.6 \times 10^{-4}$ \\
\hline B4 & 1,5-OH H-shift & $\mathrm{G}-\mathrm{OH}$ & $1.8 \times 10^{-3}$ \\
\hline \multirow{5}{*}{ B5 } & $1,5 \mathrm{H}$-shift & $E$ & 0.63 \\
\hline & $1,5 \mathrm{H}$-shift & $\mathrm{C}$ & $2.7 \times 10^{-6}$ \\
\hline & 1,6 H-shift & B & 0.13 \\
\hline & $\begin{array}{l}\text { 6-membered } \\
\text { Endoperoxide } \\
\text { formation }\end{array}$ & $\mathrm{F}$ & 0.34 \\
\hline & $\begin{array}{l}\text { 7-membered } \\
\text { Endoperoxide } \\
\text { formation }\end{array}$ & A & $1.5 \times 10^{-3}$ \\
\hline
\end{tabular}


Table S 9. Calculated reaction rate coefficients, $\mathrm{k}$, at $298.15 \mathrm{~K}$ for the unimolecular reactions of the hydroxy peroxy radicals formed from $\beta$-pinene $+\mathrm{OH}+\mathrm{O}_{2}$ (Figure S 7). All values are calculated at the B3LYP/6$31+\mathrm{G}(\mathrm{d})$ level of theory with the symmetrical Eckart tunneling approach assuming thermoneutral reactions. The abstraction/addition site refer to the structures in Figure S 6, with "-OH" referring to abstraction of the hydrogen from the hydroxy group on the specified carbon atom. The reactions highlighted in bold are the ones also treated at a higher level of theory.

\begin{tabular}{|c|c|c|c|}
\hline Peroxy radical & Reaction type & $\begin{array}{c}\text { Abstraction/addition } \\
\text { site }\end{array}$ & $\mathrm{k}\left(\mathrm{s}^{-1}\right)$ \\
\hline \multirow{5}{*}{ B1 } & 1,5 H-shift & $\mathrm{F}$ & $5.1 \times 10^{-4}$ \\
\hline & 1,6 H-shift & $\mathrm{C}$ & $6.1 \times 10^{-15}$ \\
\hline & $1,5 \mathrm{H}$-shift & $\mathrm{B}$ & $1.6 \times 10^{-3}$ \\
\hline & 1,5-OH H-shift & $\mathrm{A}-\mathrm{OH}$ & 0.61 \\
\hline & $1,7 \mathrm{H}$-shift & $\mathrm{I}$ & $1.1 \times 10^{-4}$ \\
\hline \multirow{5}{*}{ B2 } & 1,5 H-shift & $\mathbf{F}$ & $4.8 \times 10^{-4}$ \\
\hline & 1,6 H-shift & $\mathrm{C}$ & $2.7 \times 10^{-11}$ \\
\hline & 1,5 H-shift & B & $1.9 \times 10^{-2}$ \\
\hline & 1,5-OH H-shift & A-OH & 22 \\
\hline & 1,6 H-shift & $\mathrm{E}$ & $5.2 \times 10^{-6}$ \\
\hline \multirow{6}{*}{ B3 } & 1,4 H-shift & $\mathrm{F}$ & $6.3 \times 10^{-11}$ \\
\hline & $1,5 \mathrm{H}$-shift & $\mathrm{C}$ & $3.7 \times 10^{-16}$ \\
\hline & 1,4 H-shift & B & $1.9 \times 10^{-6}$ \\
\hline & 1,5 H-shift & $\mathrm{E}$ & $2.4 \times 10^{-5}$ \\
\hline & 1,4 H-shift & $\mathrm{G}$ & $6.3 \times 10^{-4}$ \\
\hline & 1,5-OH H-shift & G-OH & 0.81 \\
\hline \multirow{6}{*}{ B4 } & 1,4 H-shift & $\mathrm{F}$ & $6.3 \times 10^{-11}$ \\
\hline & $1,5 \mathrm{H}$-shift & $\mathrm{C}$ & $3.2 \times 10^{-17}$ \\
\hline & 1,4 H-shift & $\mathrm{B}$ & $5.1 \times 10^{-7}$ \\
\hline & 1,6 H-shift & $\mathrm{I}$ & $4.3 \times 10^{-6}$ \\
\hline & 1,4 H-shift & $\mathrm{G}$ & $7.0 \times 10^{-4}$ \\
\hline & 1,5-OH H-shift & G-OH & 0.87 \\
\hline \multirow{6}{*}{ B5 } & 1,5 H-shift & $\mathbf{E}$ & 4.7 \\
\hline & 1,5 H-shift & $\mathbf{C}$ & $1.3 \times 10^{-5}$ \\
\hline & 1,6 H-shift & B & 0.34 \\
\hline & 1,9-OH H-shift & $\mathrm{G}-\mathrm{OH}$ & $1.0 \times 10^{-9}$ \\
\hline & $\begin{array}{c}\text { 6-membered } \\
\text { endoperoxide } \\
\text { formation }\end{array}$ & $\mathbf{F}$ & 8.1 \\
\hline & $\begin{array}{c}\text { 7-membered } \\
\text { endoperoxide } \\
\text { formation }\end{array}$ & $\mathbf{A}$ & $2.7 \times 10^{-2}$ \\
\hline
\end{tabular}


Table S 10. CCSD(T)-F12a/VDZ-F12 (abbreviated F12) and $\omega$ B97X-D/aug-cc-pVTZ barrier heights ( $\mathrm{E}_{0}$, in $\mathrm{kcal} / \mathrm{mol})$, summed partition function ratios $\left(\mathrm{Q}_{\mathrm{TS}} / \mathrm{Q}_{\mathrm{R}}\right)$ and Eckart tunneling coefficients $(\kappa)$ used for calculating the corresponding MC-TST rate coefficients in the preceding tables for $\beta$-pinene.

\begin{tabular}{|c|c|c|c|c|c|c|c|}
\hline $\begin{array}{l}\text { Peroxy } \\
\text { radical }\end{array}$ & $\begin{array}{l}\text { Reaction } \\
\text { type }\end{array}$ & $\begin{array}{l}\text { Abstraction/ } \\
\text { addition site }\end{array}$ & $\begin{array}{c}E_{0} \\
(\mathrm{~F} 12)\end{array}$ & $\begin{array}{c}\kappa \\
(\mathrm{F} 12)\end{array}$ & $\begin{array}{c}E_{0} \\
(\omega B 97 X- \\
D)\end{array}$ & $\mathrm{Q}_{\mathrm{TS}} / \mathrm{Q}_{\mathrm{R}}$ & $\begin{array}{c}\kappa \\
(\omega \mathrm{B} 97 \mathrm{X}- \\
\mathrm{D})\end{array}$ \\
\hline B1 & 1,5-OH H-shift & $\mathrm{A}-\mathrm{OH}$ & - & - & 19.9 & 0.1169 & \\
\hline \multirow{3}{*}{ B2 } & $1,5 \mathrm{H}$-shift & $\mathrm{F}$ & - & - & 23.0 & 0.0405 & 13.0 \\
\hline & 1,5 H-shift & $\mathrm{B}$ & 20.4 & 74.4 & 21.1 & 0.1533 & 46.6 \\
\hline & 1,5-OH H-shift & $\mathrm{A}-\mathrm{OH}$ & - & - & 19.1 & 0.1073 & 2.7 \\
\hline B3 & 1,5-OH H-shift & $\mathrm{G}-\mathrm{OH}$ & - & - & 21.0 & 0.0969 & \\
\hline B4 & 1,5-OH H-shift & $\mathrm{G}-\mathrm{OH}$ & - & - & 19.9 & 0.1169 & \\
\hline \multirow{5}{*}{ B5 } & 1,5 H-shift & $\mathrm{E}$ & 19.4 & 527.6 & 19.8 & 0.0711 & 452.4 \\
\hline & 1,5 H-shift & $\mathrm{C}$ & 25.7 & 52.0 & 26.0 & 0.1620 & 32.2 \\
\hline & 1,6 H-shift & B & 19.2 & 185.5 & 19.5 & 0.0284 & 153.1 \\
\hline & $\begin{array}{c}\text { 6-membered } \\
\text { endoperoxide } \\
\text { formation } \\
\end{array}$ & $\mathrm{F}$ & 13.9 & 1.5 & 15.3 & 0.0066 & 1.5 \\
\hline & $\begin{array}{l}\text { 7-membered } \\
\text { endoperoxide } \\
\text { formation }\end{array}$ & A & 16.3 & 1.3 & 18.3 & 0.0048 & 1.3 \\
\hline
\end{tabular}


Table S 11. B3LYP/6-31+G(d) barrier heights $\left(\mathrm{E}_{0}\right.$, in $\left.\mathrm{kcal} / \mathrm{mol}\right)$, summed partition function ratios $\left(\mathrm{Q}_{\mathrm{TS}} / \mathrm{Q}_{\mathrm{R}}\right)$ and Eckart tunneling coefficients $(\kappa)$ used for calculating the corresponding MC-TST rate coefficients in the preceding tables for $\beta$-pinene.

\begin{tabular}{|c|c|c|c|c|c|}
\hline $\begin{array}{l}\text { Peroxy } \\
\text { radical }\end{array}$ & Reaction type & $\begin{array}{c}\text { Abstraction/addition } \\
\text { site } \\
\end{array}$ & $\mathrm{E}_{0}$ & $\mathrm{Q}_{\mathrm{TS}} / \mathrm{Q}_{\mathrm{R}}$ & $\kappa$ \\
\hline \multirow{5}{*}{ B1 } & $1,5 \mathrm{H}$-shift & $\mathrm{F}$ & 22.5 & 0.0341 & 71.4 \\
\hline & $1,6 \mathrm{H}$-shift & $\mathrm{C}$ & 40.6 & 0.0516 & 10420.0 \\
\hline & $1,5 \mathrm{H}$-shift & $\mathrm{B}$ & 22.5 & 0.0465 & 169.1 \\
\hline & 1,5-OH H-shift & $\mathrm{A}-\mathrm{OH}$ & 17.2 & 0.0765 & 5.0 \\
\hline & 1,7 H-shift & $\mathrm{I}$ & 23.6 & 0.0390 & 86.4 \\
\hline \multirow{5}{*}{ B2 } & 1,5 H-shift & $\mathbf{F}$ & 22.4 & 0.0315 & 59.8 \\
\hline & 1,6 H-shift & $\mathrm{C}$ & 35.0 & 0.0597 & 3278.5 \\
\hline & 1,5 H-shift & B & 21.2 & 0.0871 & 115.7 \\
\hline & 1,5-OH H-shift & A-OH & 15.7 & 0.0862 & 13.1 \\
\hline & 1,6 H-shift & $\mathrm{E}$ & 26.1 & 0.1472 & 81.1 \\
\hline \multirow{6}{*}{ B3 } & 1,4 H-shift & $\mathrm{F}$ & 35.9 & 0.1759 & 12354.0 \\
\hline & $1,5 \mathrm{H}$-shift & $\mathrm{C}$ & 43.1 & 0.0988 & 24793.0 \\
\hline & 1,4 H-shift & $\mathrm{B}$ & 31.0 & 0.2925 & 57448.0 \\
\hline & $1,5 \mathrm{H}$-shift & $\mathrm{E}$ & 24.7 & 0.1690 & 29.2 \\
\hline & 1,4 H-shift & $\mathrm{G}$ & 26.1 & 1.3521 & 966.7 \\
\hline & 1,5-OH H-shift & G-OH & 18.2 & 0.8102 & 29.2 \\
\hline \multirow{6}{*}{ B4 } & 1,4 H-shift & $\mathrm{F}$ & 36.5 & 0.253 & 23364.0 \\
\hline & 1,5 H-shift & $\mathrm{C}$ & 45.5 & 0.0829 & 127780.0 \\
\hline & 1,4 H-shift & B & 32.1 & 0.2258 & 127410.0 \\
\hline & 1,6 H-shift & $\mathrm{I}$ & 25.5 & 0.1059 & 34.0 \\
\hline & 1,4 H-shift & $\mathrm{G}$ & 25.0 & 0.2442 & 1042.9 \\
\hline & 1,5-OH H-shift & G-OH & 17.1 & 0.1246 & 4.2 \\
\hline \multirow{6}{*}{ B5 } & 1,5 H-shift & $\mathbf{E}$ & 17.8 & 0.0526 & 173.4 \\
\hline & 1,5 H-shift & $\mathbf{C}$ & 25.7 & 0.0940 & 164.2 \\
\hline & 1,6 H-shift & B & 18.9 & 0.0344 & 119.0 \\
\hline & 1,9-OH H-shift & $\mathrm{G}-\mathrm{OH}$ & 28.0 & 0.0039 & 13.1 \\
\hline & $\begin{array}{l}\text { 6-membered } \\
\text { endoperoxide } \\
\text { formation }\end{array}$ & $\mathbf{F}$ & 13.5 & 0.0079 & 1.3 \\
\hline & $\begin{array}{c}\text { 7-membered } \\
\text { endoperoxide } \\
\text { formation }\end{array}$ & $\mathbf{A}$ & 16.5 & 0.0047 & 1.2 \\
\hline
\end{tabular}




\section{S3.3 $\Delta^{3}$-Carene}

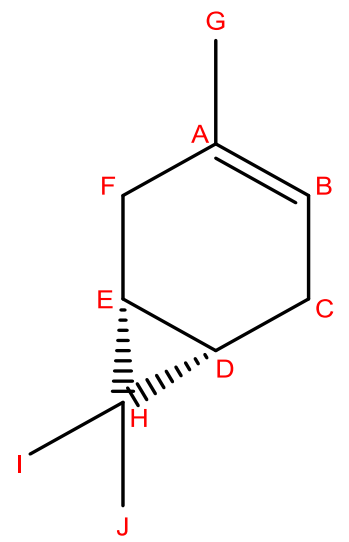

Figure S 8. Structure of $\Delta^{3}$-carene with atom labeling of the carbon atoms used to define the unimolecular reactions.

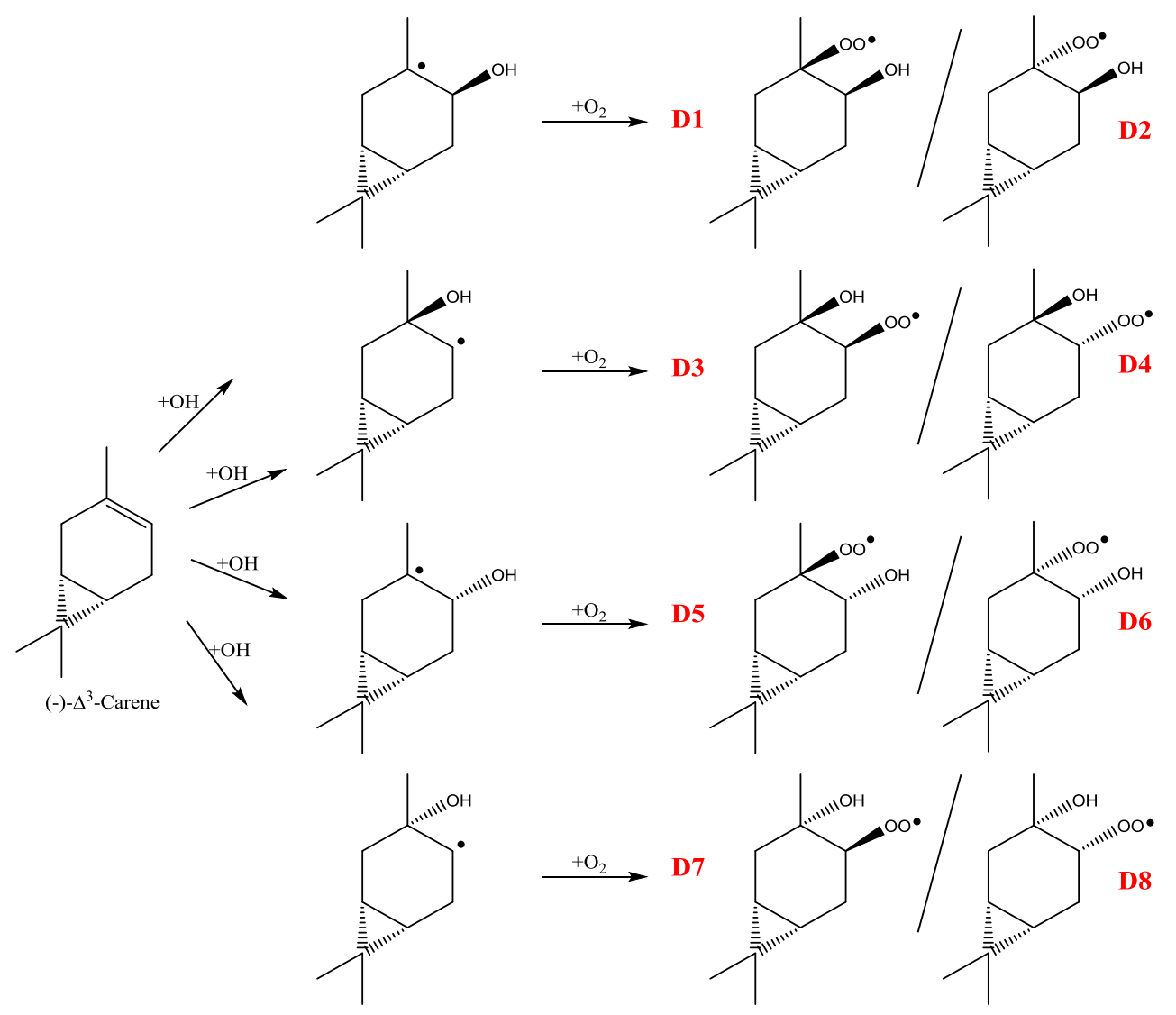

Figure S 9. Formation of the different hydroxy peroxy radicals from $\Delta^{3}$-carene $+\mathrm{OH}+\mathrm{O}_{2}$. 
Table S 12. Calculated MC-TST reaction rate coefficients, $\mathrm{k}$, at $298.15 \mathrm{~K}$ for the unimolecular reactions of the hydroxy peroxy radicals formed from $\Delta 3$-carene $+\mathrm{OH}+\mathrm{O}_{2}$ (Figure $\mathrm{S} 9$ ). All values are calculated at the $\omega B 97 X-D / a u g-c c-p V T Z$ level of theory with tunneling based on IRC end-points. The abstraction/addition site refer to the structures in Figure S 8, with "-OH" referring to abstraction of the hydrogen from the hydroxy group on the specified carbon atom.

\begin{tabular}{|c|c|c|c|}
\hline Peroxy radical & Reaction type & $\begin{array}{c}\text { Abstraction/addition } \\
\text { site }\end{array}$ & $\mathrm{k}\left(\mathrm{s}^{-1}\right)$ \\
\hline D1 & $1,5-\mathrm{OH}$ H-shift & B-OH & $7.1 \times 10^{-4}$ \\
\hline D2 & $1,5-\mathrm{OH}$ H-shift & B-OH & $2.6 \times 10^{-4}$ \\
\hline D3 & $1,5-\mathrm{OH}$ H-shift & A-OH & $3.7 \times 10^{-4}$ \\
\hline D4 & $1,5-\mathrm{OH}$ H-shift & A-OH & $2.9 \times 10^{-5}$ \\
\hline \multirow{2}{*}{ D5 } & 1,4 H-shift & B & $1.4 \times 10^{-4}$ \\
\hline D6 & $1,5-\mathrm{OH}$ H-shift & B-OH & $3.1 \times 10^{-5}$ \\
\hline D7 & $1,5-\mathrm{OH}$ H-shift & B-OH & $9.2 \times 10^{-4}$ \\
\hline D8 & $1,5-\mathrm{OH}$ H-shift & A-OH & $4.8 \times 10^{-3}$ \\
\hline
\end{tabular}


Table S 13. Calculated reaction rate coefficients, $\mathrm{k}$, at $298.15 \mathrm{~K}$ for the unimolecular reactions of the hydroxy peroxy radicals formed from $\Delta^{3}$-carene $+\mathrm{OH}+\mathrm{O}_{2}$ (Figure $\mathrm{S}$ 9). All values are calculated at the B3LYP/6$31+\mathrm{G}(\mathrm{d})$ level of theory with the symmetrical Eckart tunneling approach assuming thermoneutral reactions. The abstraction/addition site refer to the structures in Figure S 8, with "-OH" referring to abstraction of the hydrogen from the hydroxy group on the specified carbon atom. The reactions highlighted in bold are the ones also treated at a higher level of theory.

\begin{tabular}{|c|c|c|c|}
\hline Peroxy radical & Reaction type & $\begin{array}{l}\text { Abstraction/addition } \\
\text { site }\end{array}$ & $\mathrm{k}\left(\mathrm{s}^{-1}\right)$ \\
\hline \multirow{4}{*}{ D1 } & 1,5 H-shift & $\mathrm{E}$ & $5.0 \times 10^{-11}$ \\
\hline & 1,5-OH H-shift & B-OH & 0.85 \\
\hline & 1,5 H-shift & $\mathrm{C}$ & $1.6 \times 10^{-4}$ \\
\hline & 1,6 H-shift & $\mathrm{D}$ & $9.1 \times 10^{-15}$ \\
\hline \multirow{4}{*}{ D2 } & 1,4 H-shift & $\mathrm{B}$ & $3.0 \times 10^{-5}$ \\
\hline & 1,5-OH H-shift & B-OH & 0.22 \\
\hline & 1,5 H-shift & $\mathrm{C}$ & $5.5 \times 10^{-9}$ \\
\hline & 1,7 H-shift & I & $5.8 \times 10^{-7}$ \\
\hline \multirow{4}{*}{ D3 } & $1,5 \mathrm{H}$-shift & $\mathrm{F}$ & $4.2 \times 10^{-10}$ \\
\hline & 1,5-OH H-shift & A-OH & 0.46 \\
\hline & $1,5 \mathrm{H}$-shift & $\mathrm{D}$ & $4.5 \times 10^{-12}$ \\
\hline & 1,6 H-shift & $\mathrm{E}$ & $1.9 \times 10^{-16}$ \\
\hline \multirow{3}{*}{ D4 } & 1,5 H-shift & $\mathrm{F}$ & $4.1 \times 10^{-8}$ \\
\hline & 1,5-OH H-shift & $\mathrm{A}-\mathrm{OH}$ & $2.9 \times 10^{-2}$ \\
\hline & 1,7 H-shift & I & $1.7 \times 10^{-5}$ \\
\hline \multirow{5}{*}{ D5 } & 1,4 H-shift & B & $3.3 \times 10^{-4}$ \\
\hline & 1,5 H-shift & $\mathrm{E}$ & $1.2 \times 10^{-10}$ \\
\hline & 1,5-OH H-shift & B-OH & $2.6 \times 10^{-2}$ \\
\hline & 1,5 H-shift & $\mathrm{C}$ & $9.2 \times 10^{-7}$ \\
\hline & 1,6 H-shift & $\mathrm{D}$ & $3.1 \times 10^{-15}$ \\
\hline \multirow{3}{*}{ D6 } & 1,5-OH H-shift & B-OH & 2.8 \\
\hline & 1,5 H-shift & $\mathrm{C}$ & $2.4 \times 10^{-6}$ \\
\hline & 1,7 H-shift & $\mathrm{I}$ & $1.0 \times 10^{-5}$ \\
\hline \multirow{4}{*}{ D7 } & 1,5 H-shift & $\mathrm{F}$ & $2.6 \times 10^{-8}$ \\
\hline & 1,5-OH H-shift & A-OH & 4.9 \\
\hline & 1,5 H-shift & $\mathrm{D}$ & $3.9 \times 10^{-12}$ \\
\hline & $1,6 \mathrm{H}$-shift & $\mathrm{E}$ & $2.8 \times 10^{-17}$ \\
\hline \multirow{3}{*}{ D8 } & 1,5 H-shift & $\mathrm{F}$ & $1.8 \times 10^{-7}$ \\
\hline & 1,5-OH H-shift & A-OH & 4.5 \\
\hline & $1,7 \mathrm{H}$-shift & I & $6.5 \times 10^{-6}$ \\
\hline
\end{tabular}


Table S 14. $\omega \mathrm{B} 97 \mathrm{X}-\mathrm{D} /$ aug-cc-pVTZ barrier heights $\left(\mathrm{E}_{0}\right.$, in $\left.\mathrm{kcal} / \mathrm{mol}\right)$, summed partition function ratios $\left(\mathrm{Q}_{\mathrm{TS}} / \mathrm{Q}_{\mathrm{R}}\right)$ and Eckart tunneling coefficients $(\kappa)$ used for calculating the corresponding MC-TST rate coefficients in the preceding tables for $\Delta^{3}$-carene.

\begin{tabular}{|c|c|c|c|c|c|}
\hline Peroxy radical & Reaction type & $\begin{array}{c}\text { Abstraction/ } \\
\text { addition site }\end{array}$ & $\begin{array}{c}\mathrm{E}_{0} \\
(\omega \mathrm{B} 97 \mathrm{X}-\mathrm{D})\end{array}$ & $\mathrm{Q}_{\mathrm{TS}} / \mathrm{Q}_{\mathrm{R}}$ & $\begin{array}{c}\kappa \\
(\omega \mathrm{B} 97 \mathrm{X}-\mathrm{D})\end{array}$ \\
\hline D1 & $1,5-\mathrm{OH}$ H-shift & $\mathrm{B}-\mathrm{OH}$ & 21.5 & $5.63 \times 10^{-1}$ & 1.2 \\
\hline D2 & $1,5-\mathrm{OH}$ H-shift & B-OH & 21.3 & $1.23 \times 10^{-1}$ & 1.5 \\
\hline D3 & $1,5-\mathrm{OH}$ H-shift & A-OH & 21.3 & $1.82 \times 10^{-1}$ & 1.3 \\
\hline D4 & $1,5-\mathrm{OH}$ H-shift & A-OH & 23.1 & $1.99 \times 10^{-1}$ & 2.0 \\
\hline \multirow{2}{*}{ D5 } & 1,4 H-shift & B & 26.1 & $6.16 \times 10^{-1}$ & 504.6 \\
\cline { 2 - 6 } & $1,5-\mathrm{OH}$ H-shift & B-OH & 23.0 & $1.61 \times 10^{-1}$ & 2.1 \\
\hline D6 & $1,5-\mathrm{OH}$ H-shift & B-OH & 20.4 & $2.36 \times 10^{-1}$ & 0.5 \\
\hline D7 & $1,5-\mathrm{OH}$ H-shift & A-OH & 19.7 & $2.03 \times 10^{-1}$ & 1.0 \\
\hline D8 & $1,5-\mathrm{OH}$ H-shift & A-OH & 19.5 & $1.85 \times 10^{-1}$ & 1.0 \\
\hline
\end{tabular}


Table S 15. B3LYP/6-31+G(d) barrier heights $\left(\mathrm{E}_{0}\right.$, in kcal/mol), summed partition function ratios $\left(\mathrm{Q}_{\mathrm{TS}} / \mathrm{Q}_{\mathrm{R}}\right)$ and Eckart tunneling coefficients $(\kappa)$ used for calculating the corresponding MC-TST rate coefficients in the preceding tables $\Delta^{3}$-carene.

\begin{tabular}{|c|c|c|c|c|c|}
\hline Peroxy radical & Reaction type & $\begin{array}{l}\text { Abstraction/ } \\
\text { addition site }\end{array}$ & $\begin{array}{c}\mathrm{E}_{0} \\
(\mathrm{~B} 3 \mathrm{LYP})\end{array}$ & $\mathrm{Q}_{\mathrm{TS}} / \mathrm{Q}_{\mathrm{R}}$ & $\begin{array}{c}\kappa \\
(\mathrm{B} 3 \mathrm{LYP})\end{array}$ \\
\hline \multirow{4}{*}{ D1 } & $1,5 \mathrm{H}$-shift & $\mathrm{E}$ & 34.9 & $2.28 \times 10^{-1}$ & 1394.2 \\
\hline & 1,5-OH H-shift & $\mathrm{B}-\mathrm{OH}$ & 18.4 & $5.37 \times 10^{-1}$ & 7.7 \\
\hline & $1,5 \mathrm{H}$-shift & $\mathrm{C}$ & 25.2 & $2.25 \times 10^{-1}$ & 325.9 \\
\hline & 1,6 H-shift & $\mathrm{D}$ & 42.3 & $1.13 \times 10^{-1}$ & 134366.7 \\
\hline \multirow{4}{*}{ D2 } & 1,4 H-shift & $\mathrm{B}$ & 26.8 & $3.13 \times 10^{-1}$ & 652.5 \\
\hline & 1,5-OH H-shift & $\mathrm{B}-\mathrm{OH}$ & 18.4 & $1.13 \times 10^{-1}$ & 9.8 \\
\hline & 1,5 H-shift & $\mathrm{C}$ & 31.8 & $1.94 \times 10^{-1}$ & 1007.6 \\
\hline & 1,7 H-shift & I & 28.0 & $1.74 \times 10^{-1}$ & 174.5 \\
\hline \multirow{4}{*}{ D3 } & $1,5 \mathrm{H}$-shift & $\mathrm{F}$ & 33.0 & $5.72 \times 10^{-2}$ & 1703.3 \\
\hline & 1,5-OH H-shift & $\mathrm{A}-\mathrm{OH}$ & 17.8 & $1.08 \times 10^{-1}$ & 7.6 \\
\hline & 1,5 H-shift & $\mathrm{D}$ & 35.6 & $5.51 \times 10^{-2}$ & 1698.8 \\
\hline & 1,6 H-shift & $\mathrm{E}$ & 43.9 & $2.52 \times 10^{-2}$ & 173943.3 \\
\hline \multirow{3}{*}{ D4 } & 1,5 H-shift & $\mathrm{F}$ & 30.6 & $1.53 \times 10^{-1}$ & 1130.8 \\
\hline & 1,5-OH H-shift & $\mathrm{A}-\mathrm{OH}$ & 19.8 & $1.30 \times 10^{-1}$ & 11.9 \\
\hline & 1,7 H-shift & I & 26.0 & $1.96 \times 10^{-1}$ & 155.9 \\
\hline \multirow{5}{*}{ D5 } & 1,4 H-shift & $\mathrm{B}$ & 25.8 & $5.38 \times 10^{-1}$ & 840.1 \\
\hline & $1,5 \mathrm{H}$-shift & $\mathrm{E}$ & 34.4 & $1.53 \times 10^{-1}$ & 2056.8 \\
\hline & 1,5-OH H-shift & $\mathrm{B}-\mathrm{OH}$ & 20.0 & $1.54 \times 10^{-1}$ & 13.3 \\
\hline & 1,5 H-shift & $\mathrm{C}$ & 29.6 & $1.99 \times 10^{-1}$ & 3526.5 \\
\hline & 1,6 H-shift & $\mathrm{D}$ & 43.8 & $1.74 \times 10^{-1}$ & 345816.4 \\
\hline \multirow{3}{*}{ D6 } & 1,5-OH H-shift & $\mathrm{B}-\mathrm{OH}$ & 17.0 & $2.73 \times 10^{-1}$ & 4.8 \\
\hline & 1,5 H-shift & $\mathrm{C}$ & 26.7 & $7.80 \times 10^{-2}$ & 187.9 \\
\hline & 1,7 H-shift & I & 25.6 & $7.66 \times 10^{-2}$ & 127.3 \\
\hline \multirow{4}{*}{ D7 } & 1,5 H-shift & $\mathrm{F}$ & 31.7 & $1.33 \times 10^{-1}$ & 4956.9 \\
\hline & 1,5-OH H-shift & $\mathrm{A}-\mathrm{OH}$ & 16.6 & $1.46 \times 10^{-1}$ & 7.8 \\
\hline & 1,5 H-shift & $\mathrm{D}$ & 37.0 & $2.38 \times 10^{-1}$ & 3504.2 \\
\hline & 1,6 H-shift & $\mathrm{E}$ & 47.0 & $1.57 \times 10^{-1}$ & 873421.0 \\
\hline \multirow{3}{*}{ D8 } & 1,5 H-shift & $\mathrm{F}$ & 28.4 & $6.35 \times 10^{-2}$ & 278.6 \\
\hline & 1,5-OH H-shift & $\mathrm{A}-\mathrm{OH}$ & 16.6 & $1.21 \times 10^{-1}$ & 8.9 \\
\hline & 1,7 H-shift & $\mathrm{I}$ & 25.5 & $4.85 \times 10^{-2}$ & 102.4 \\
\hline
\end{tabular}




\section{S3.4 Camphene}

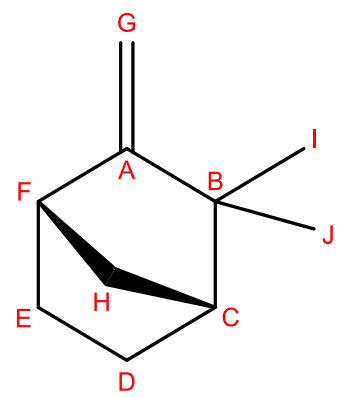

Figure S 10. Structure of camphene with atom labeling of the carbon atoms used to define the unimolecular reactions.
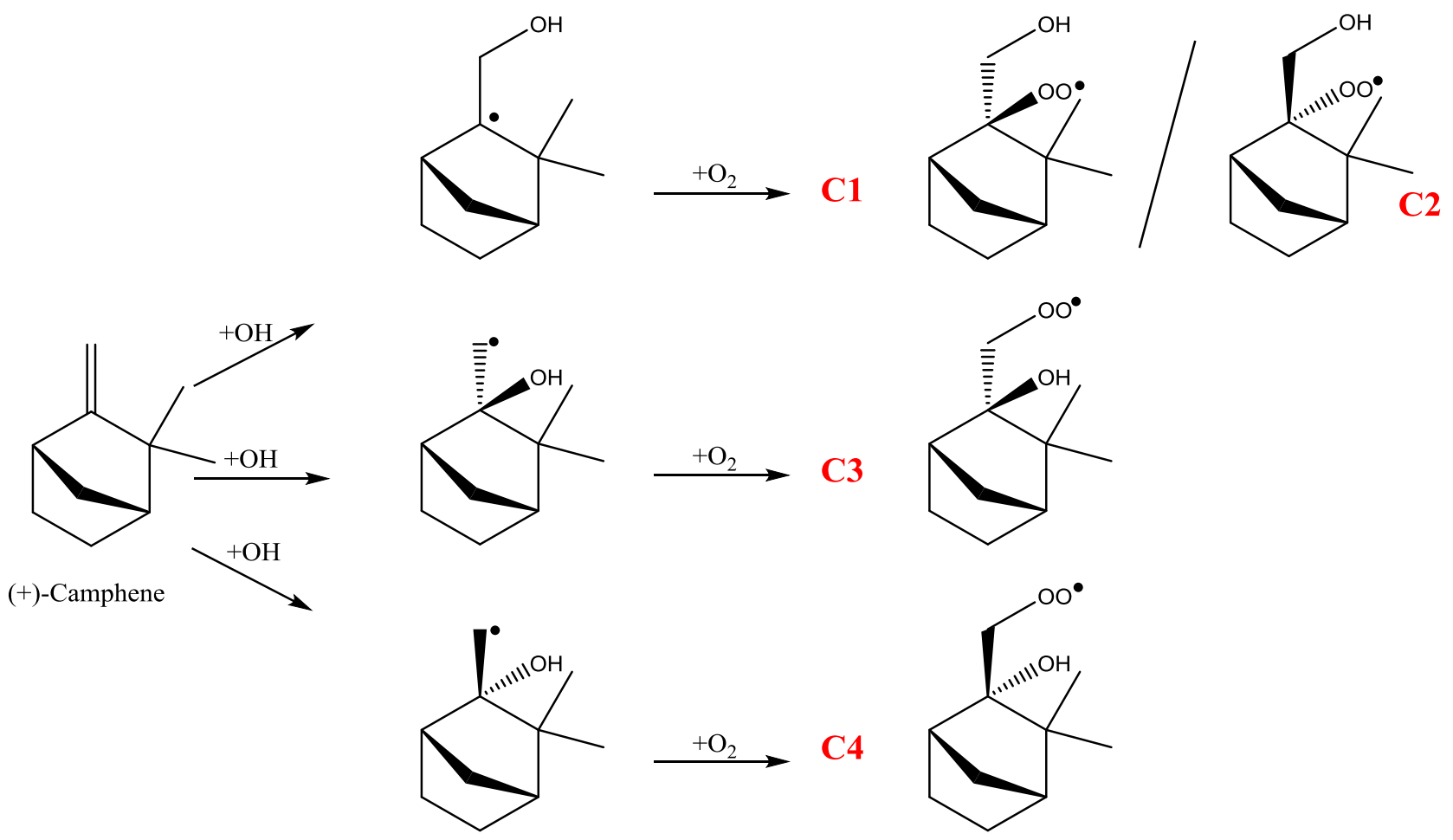

Figure S 11. Formation of the different hydroxy peroxy radicals from camphene $+\mathrm{OH}+\mathrm{O}_{2}$. 
Table S 16. Calculated MC-TST reaction rate coefficients, $\mathrm{k}$, at $298.15 \mathrm{~K}$ for the unimolecular reactions of the hydroxy peroxy radicals formed from camphene $+\mathrm{OH}+\mathrm{O}_{2}$ (Figure $\mathrm{S} 11$ ). All values are calculated at the $\omega B 97 X-D / a u g-c c-p V T Z$ level of theory with tunneling based on IRC end-points. The abstraction/addition site refer to the structures in Figure S 10, with "-OH" referring to abstraction of the hydrogen from the hydroxy group on the specified carbon atom.

\begin{tabular}{|c|c|c|c|}
\hline \multirow{2}{*}{ Peroxy radical } & Reaction type & $\begin{array}{c}\text { Abstraction/addition } \\
\text { site }\end{array}$ & $\mathrm{k}\left(\mathrm{s}^{-1}\right)$ \\
\hline \multirow{2}{*}{$\mathrm{C} 1$} & $1,4 \mathrm{H}$-shift & $\mathrm{G}$ & $3.3 \times 10^{-6}$ \\
\cline { 2 - 4 } & $1,5-\mathrm{OH}$ H-shift & $\mathrm{G}-\mathrm{OH}$ & $6.5 \times 10^{-4}$ \\
\cline { 2 - 4 } & $1,5 \mathrm{H}$-shift & $\mathrm{I}$ & $2.0 \times 10^{-4}$ \\
\hline $\mathrm{C} 2$ & $1,5-\mathrm{OH}$ H-shift & $\mathrm{G}-\mathrm{OH}$ & $1.0 \times 10^{-2}$ \\
\hline $\mathrm{C} 3$ & $1,5-\mathrm{OH}$ H-shift & $\mathrm{A}-\mathrm{OH}$ & $5.1 \times 10^{-3}$ \\
\hline $\mathrm{C} 4$ & $1,5-\mathrm{OH}$ H-shift & $\mathrm{A}-\mathrm{OH}$ & $2.2 \times 10^{-2}$ \\
\hline
\end{tabular}


Table S 17. Calculated reaction rate coefficients, $\mathrm{k}$, at $298.15 \mathrm{~K}$ for the unimolecular reactions of the hydroxy peroxy radicals formed from camphene $+\mathrm{OH}+\mathrm{O}_{2}$ (Figure $\mathrm{S} 11$ ). All values are calculated at the B3LYP/6$31+\mathrm{G}(\mathrm{d})$ level of theory with the symmetrical Eckart tunneling approach assuming thermoneutral reactions. The abstraction/addition site refer to the structures in Figure S 10, with "-OH" referring to abstraction of the hydrogen from the hydroxy group on the specified carbon atom. The reactions highlighted in bold are the ones also treated at a higher level of theory.

\begin{tabular}{|c|c|c|c|}
\hline Peroxy radical & Reaction type & $\begin{array}{c}\text { Abstraction/addition } \\
\text { site }\end{array}$ & $\mathrm{k}\left(\mathrm{s}^{-1}\right)$ \\
\hline \multirow{5}{*}{$\mathrm{C} 1$} & 1,4 H-shift & $\mathrm{F}$ & $1.5 \times 10^{-10}$ \\
\hline & 1,4 H-shift & $\mathbf{G}$ & $1.0 \times 10^{-5}$ \\
\hline & 1,5 H-shift & $\mathrm{H}$ & $9.7 \times 10^{-10}$ \\
\hline & 1,5 H-shift & I & $6.3 \times 10^{-4}$ \\
\hline & 1,5-OH H-shift & G-OH & 0.33 \\
\hline \multirow{6}{*}{$\mathrm{C} 2$} & 1,4 H-shift & $\mathrm{F}$ & $1.5 \times 10^{-16}$ \\
\hline & 1,4 H-shift & $\mathrm{G}$ & $2.5 \times 10^{-5}$ \\
\hline & 1,5 H-shift & $\mathrm{E}$ & $7.9 \times 10^{-5}$ \\
\hline & 1,5 H-shift & I & $4.6 \times 10^{-4}$ \\
\hline & 1,5-OH H-shift & G-OH & 4.5 \\
\hline & 1,6 H-shift & $\mathrm{D}$ & $4.1 \times 10^{-13}$ \\
\hline \multirow{5}{*}{$\mathrm{C} 3$} & 1,5 H-shift & $\mathrm{F}$ & $2.7 \times 10^{-9}$ \\
\hline & 1,5-OH H-shift & A-OH & 4.9 \\
\hline & 1,6 H-shift & $\mathrm{E}$ & $1.1 \times 10^{-4}$ \\
\hline & 1,6 H-shift & $\mathrm{I}$ & $7.1 \times 10^{-6}$ \\
\hline & 1,7 H-shift & $\mathrm{D}$ & $1.0 \times 10^{-11}$ \\
\hline \multirow{4}{*}{$\mathrm{C} 4$} & 1,5 H-shift & $\mathrm{F}$ & $4.5 \times 10^{-6}$ \\
\hline & 1,5-OH H-shift & A-OH & 19 \\
\hline & 1,6 H-shift & $\mathrm{H}$ & $7.9 \times 10^{-9}$ \\
\hline & 1,6 H-shift & $\mathrm{I}$ & $1.7 \times 10^{-5}$ \\
\hline
\end{tabular}


Table S 18. $\omega \mathrm{B} 97 \mathrm{X}-\mathrm{D} /$ aug-cc-pVTZ barrier heights $\left(\mathrm{E}_{0}\right.$, in $\left.\mathrm{kcal} / \mathrm{mol}\right)$, summed partition function ratios $\left(\mathrm{Q}_{\mathrm{TS}} / \mathrm{Q}_{\mathrm{R}}\right)$ and Eckart tunneling coefficients $(\kappa)$ used for calculating the corresponding MC-TST rate coefficients in the preceding tables for camphene.

\begin{tabular}{|c|c|c|c|c|c|}
\hline \multirow{2}{*}{ Peroxy radical } & Reaction type & $\begin{array}{c}\text { Abstraction/ } \\
\text { addition site }\end{array}$ & $\begin{array}{c}\mathrm{E}_{0}(\omega \mathrm{B} 97 \mathrm{X}- \\
\mathrm{D})\end{array}$ & $\mathrm{Q}_{\mathrm{TS}} / \mathrm{Q}_{\mathrm{R}}$ & $\begin{array}{c}\kappa \\
(\omega \mathrm{B} 97 \mathrm{X}-\mathrm{D})\end{array}$ \\
\hline \multirow{3}{*}{$\mathrm{C} 1$} & $1,4 \mathrm{H}$-shift & $\mathrm{G}$ & 27.7 & $1.76 \times 10^{-1}$ & 571.2 \\
\cline { 2 - 6 } & $1,5-\mathrm{OH}$ H-shift & $\mathrm{G}-\mathrm{OH}$ & 20.5 & $1.20 \times 10^{-1}$ & 1.0 \\
\cline { 2 - 6 } & $1,5 \mathrm{H}-$-shift & $\mathrm{I}$ & 23.0 & $1.38 \times 10^{-1}$ & 16.2 \\
\hline $\mathrm{C} 2$ & $1,5-\mathrm{OH}$ H-shift & $\mathrm{G}-\mathrm{OH}$ & 18.8 & $9.75 \times 10^{-2}$ & 1.0 \\
\hline $\mathrm{C} 3$ & $1,5-\mathrm{OH}$ H-shift & $\mathrm{A}-\mathrm{OH}$ & 19.6 & $1.52 \times 10^{-1}$ & 1.2 \\
\hline $\mathrm{C} 4$ & $1,5-\mathrm{OH}$ H-shift & A-OH & 18.7 & $1.56 \times 10^{-1}$ & 1.1 \\
\hline
\end{tabular}


Table S 19. B3LYP/6-31+G(d) barrier heights $\left(\mathrm{E}_{0}\right.$, in $\left.\mathrm{kcal} / \mathrm{mol}\right)$, summed partition function ratios $\left(\mathrm{Q}_{\mathrm{TS}} / \mathrm{Q}_{\mathrm{R}}\right)$ and Eckart tunneling coefficients $(\kappa)$ used for calculating the corresponding MC-TST rate coefficients in the preceding tables for camphene.

\begin{tabular}{|c|c|c|c|c|c|}
\hline Peroxy radical & Reaction type & $\begin{array}{l}\text { Abstraction/ } \\
\text { addition site }\end{array}$ & $\begin{array}{c}\mathrm{E}_{0} \\
(\mathrm{~B} 3 \mathrm{LYP})\end{array}$ & $\mathrm{Q}_{\mathrm{TS}} / \mathrm{Q}_{\mathrm{R}}$ & $\begin{array}{c}\kappa \\
(\mathrm{B} 3 \mathrm{LYP})\end{array}$ \\
\hline \multirow{5}{*}{$\mathrm{C} 1$} & 1,4 H-shift & $\mathrm{F}$ & 36.5 & $3.72 \times 10^{-1}$ & 38009.5 \\
\hline & 1,4 H-shift & $\mathrm{G}$ & 27.8 & $2.71 \times 10^{-1}$ & 1491.2 \\
\hline & $1,5 \mathrm{H}$-shift & $\mathrm{H}$ & 31.6 & $2.41 \times 10^{-1}$ & 86.8 \\
\hline & 1,5 H-shift & I & 23.2 & $1.74 \times 10^{-1}$ & 62.2 \\
\hline & 1,5-OH H-shift & G-OH & 17.7 & $1.49 \times 10^{-1}$ & 3.3 \\
\hline \multirow{6}{*}{$\mathrm{C} 2$} & 1,4 H-shift & $\mathrm{F}$ & 46.3 & $1.19 \times 10^{-1}$ & 1856606.8 \\
\hline & 1,4 H-shift & $\mathrm{G}$ & 27.1 & $2.78 \times 10^{-1}$ & 1017.9 \\
\hline & 1,5 H-shift & $\mathrm{E}$ & 23.9 & $1.09 \times 10^{-1}$ & 41.1 \\
\hline & 1,5 H-shift & I & 23.6 & $1.43 \times 10^{-1}$ & 96.3 \\
\hline & 1,5-OH H-shift & $\mathrm{G}-\mathrm{OH}$ & 16.0 & $9.72 \times 10^{-2}$ & 4.0 \\
\hline & 1,6 H-shift & $\mathrm{D}$ & 37.8 & $8.73 \times 10^{-2}$ & 4018.4 \\
\hline \multirow{5}{*}{$\mathrm{C} 3$} & 1,5 H-shift & $\mathrm{F}$ & 31.6 & $4.54 \times 10^{-2}$ & 1405.3 \\
\hline & 1,5-OH H-shift & $\mathrm{A}-\mathrm{OH}$ & 16.1 & $1.55 \times 10^{-1}$ & 3.4 \\
\hline & 1,6 H-shift & $\mathrm{E}$ & 24.3 & $1.21 \times 10^{-1}$ & 100.8 \\
\hline & 1,6 H-shift & I & 25.9 & $5.62 \times 10^{-2}$ & 188.3 \\
\hline & 1,7 H-shift & $\mathrm{D}$ & 34.5 & $7.57 \times 10^{-2}$ & 451.8 \\
\hline \multirow{4}{*}{$\mathrm{C} 4$} & 1,5 H-shift & $\mathrm{F}$ & 25.2 & $4.64 \times 10^{-2}$ & 47.8 \\
\hline & 1,5-OH H-shift & $\mathrm{A}-\mathrm{OH}$ & 15.2 & $1.04 \times 10^{-1}$ & 4.3 \\
\hline & 1,6 H-shift & $\mathrm{H}$ & 29.2 & $9.15 \times 10^{-2}$ & 34.8 \\
\hline & 1,6 H-shift & $\mathrm{I}$ & 25.0 & $4.29 \times 10^{-2}$ & 140.5 \\
\hline
\end{tabular}




\section{S3.5 Limonene}

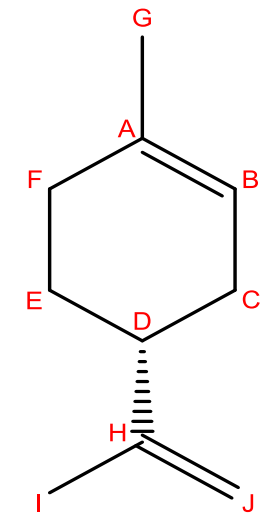

Figure S 12. Structure of limonene with atom labeling of the carbon atoms used to define the unimolecular reactions.

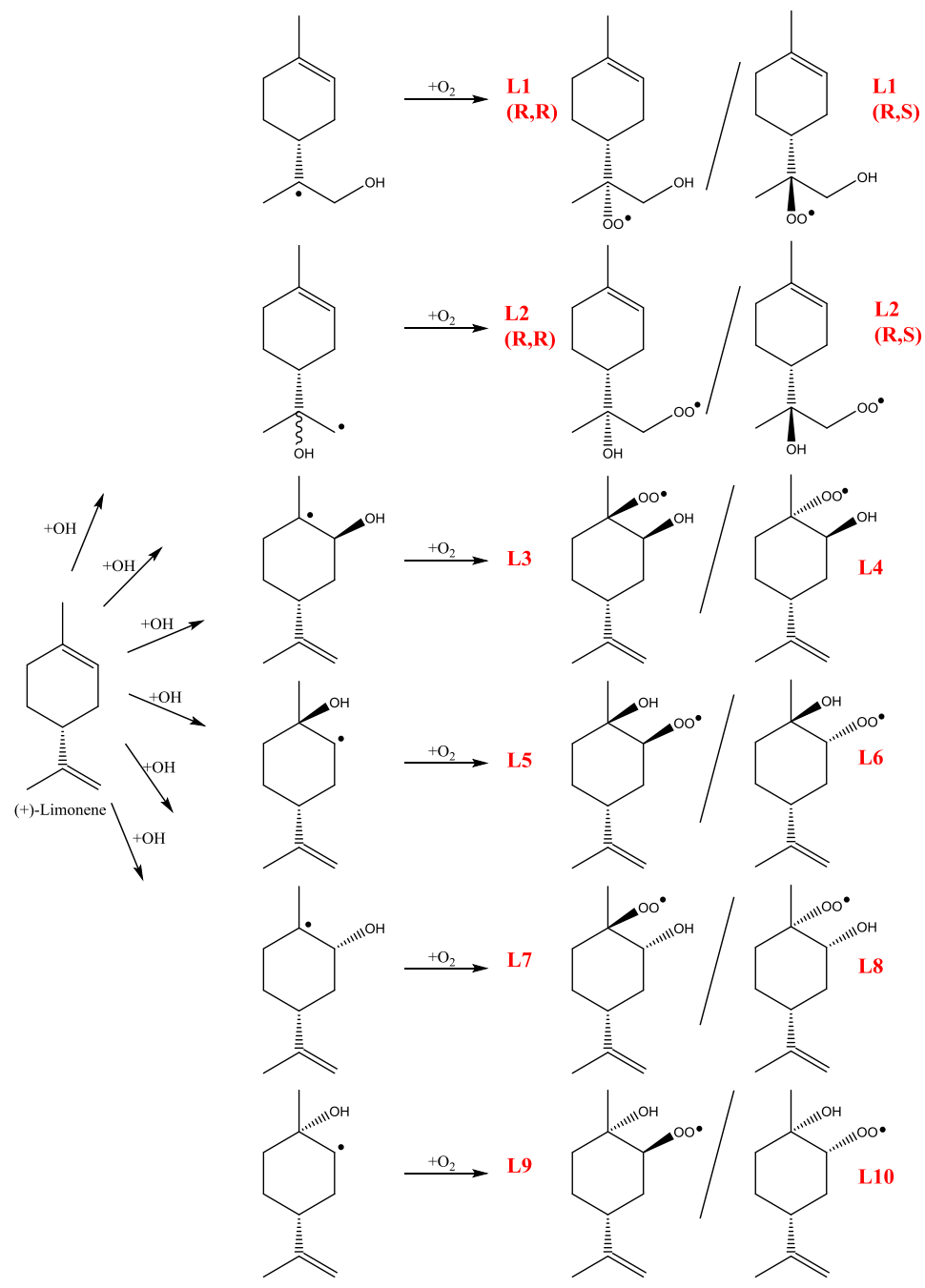

Figure $\mathrm{S}$ 13. Formation of the different hydroxy peroxy radicals from limonene $+\mathrm{OH}+\mathrm{O}_{2}$. 
Table S 20. Calculated MC-TST reaction rate coefficients, $\mathrm{k}$, at $298.15 \mathrm{~K}$ for the unimolecular reactions of the hydroxy peroxy radicals formed from limonene $+\mathrm{OH}+\mathrm{O}_{2}$ (Figure $\mathrm{S} 13$ ). The rate coefficients are calculated using the approach by Møller et al. ${ }^{1}$ The electronic energies of the lowest-energy conformers and IRC end-points are calculated at the CCSD(T)-F12a/VDZ-F12 level while the remaining parameters are calculated at the $\omega$ B97X-D/aug-cc-pVTZ level. Tunneling is based on IRC end-points. The

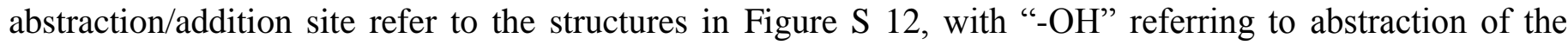
hydrogen from the hydroxy group on the specified carbon atom.

\begin{tabular}{|c|c|c|c|}
\hline Peroxy radical & Reaction type & $\begin{array}{c}\text { Abstraction/addition } \\
\text { site }\end{array}$ & $\mathrm{k}\left(\mathrm{s}^{-1}\right)$ \\
\hline \multirow{4}{*}{ L1 } & 1,5 H-shift & $\mathrm{C}$ & $\begin{array}{l}4.0(\mathrm{R}, \mathrm{R}) \\
0.53(\mathrm{R}, \mathrm{S})\end{array}$ \\
\hline & 1,6 H-shift & $\mathrm{F}$ & $\begin{array}{l}0.20(\mathrm{R}, \mathrm{R}) \\
0.69(\mathrm{R}, \mathrm{S})\end{array}$ \\
\hline & $\begin{array}{c}\text { 6-membered } \\
\text { endoperoxide } \\
\text { formation }\end{array}$ & B & $\begin{array}{c}3.9(\mathrm{R}, \mathrm{R}) \\
0.93(\mathrm{R}, \mathrm{S})\end{array}$ \\
\hline & $\begin{array}{c}\text { 7-membered } \\
\text { endoperoxide } \\
\text { formation }\end{array}$ & A & $\begin{array}{l}3.6 \times 10^{-2}(\mathrm{R}, \mathrm{R}) \\
1.2 \times 10^{-2}(\mathrm{R}, \mathrm{S})\end{array}$ \\
\hline \multirow{6}{*}{ L2 } & 1,5 H-shift & $\mathrm{D}$ & $\begin{array}{c}0.31(\mathrm{R}, \mathrm{R}) \\
6.9 \times 10^{-2}(\mathrm{R}, \mathrm{S})\end{array}$ \\
\hline & 1,6 H-shift & $\mathrm{E}$ & $\begin{array}{l}7.0 \times 10^{-3}(\mathrm{R}, \mathrm{R}) \\
1.2 \times 10^{-3}(\mathrm{R}, \mathrm{S})\end{array}$ \\
\hline & 1,6 H-shift & $\mathrm{C}$ & $\begin{array}{l}30(\mathrm{R}, \mathrm{R}) \\
36(\mathrm{R}, \mathrm{S})\end{array}$ \\
\hline & 1,7 H-shift & $\mathrm{F}$ & $\begin{array}{c}0.17(\mathrm{R}, \mathrm{R}) \\
8.1 \times 10^{-2}(\mathrm{R}, \mathrm{S})\end{array}$ \\
\hline & $\begin{array}{c}\text { 7-membered } \\
\text { endoperoxide } \\
\text { formation }\end{array}$ & B & $\begin{array}{l}2.1 \times 10^{-2}(\mathrm{R}, \mathrm{R}) \\
6.7 \times 10^{-2}(\mathrm{R}, \mathrm{S})\end{array}$ \\
\hline & $\begin{array}{l}\text { 8-membered } \\
\text { endoperoxide } \\
\text { formation }\end{array}$ & $\mathrm{A}$ & $\begin{array}{c}0.17(\mathrm{R}, \mathrm{R}) \\
3.8 \times 10^{-2}(\mathrm{R}, \mathrm{S})\end{array}$ \\
\hline L5 & 1,5 H-shift & $\mathrm{D}$ & $7.8 \times 10^{-3}$ \\
\hline
\end{tabular}


Table S 21. Calculated MC-TST reaction rate coefficients, $\mathrm{k}$, at $298.15 \mathrm{~K}$ for the unimolecular reactions of the hydroxy peroxy radicals formed from limonene $+\mathrm{OH}+\mathrm{O}_{2}$ (Figure $\mathrm{S} 13$ ). All values are calculated at the $\omega$ B97X-D/aug-cc-pVTZ level of theory with tunneling based on IRC end-points. The abstraction/addition site refer to the structures in Figure S 12, with "- $\mathrm{OH}$ " referring to abstraction of the hydrogen from the hydroxy group on the specified carbon atom.

\begin{tabular}{|c|c|c|c|}
\hline Peroxy radical & Reaction type & $\begin{array}{c}\text { Abstraction/addition } \\
\text { site }\end{array}$ & $\mathrm{k}\left(\mathrm{s}^{-1}\right)$ \\
\hline \multirow{5}{*}{ L1 } & 1,5-OH H-shift & $\mathrm{J}-\mathrm{OH}$ & $\begin{array}{l}2.2 \times 10^{-4}(\mathrm{R}, \mathrm{R}) \\
8.2 \times 10^{-5}(\mathrm{R}, \mathrm{S})\end{array}$ \\
\hline & 1,5 H-shift & $\mathrm{C}$ & $\begin{array}{c}1.7(\mathrm{R}, \mathrm{R}) \\
0.54(\mathrm{R}, \mathrm{S})\end{array}$ \\
\hline & 1,6 H-shift & $\mathrm{F}$ & $\begin{array}{l}0.14(\mathrm{R}, \mathrm{R}) \\
0.26(\mathrm{R}, \mathrm{S})\end{array}$ \\
\hline & $\begin{array}{l}\text { 6-membered } \\
\text { endoperoxide } \\
\text { formation }\end{array}$ & B & $\begin{array}{l}0.79(\mathrm{R}, \mathrm{R}) \\
0.22(\mathrm{R}, \mathrm{S})\end{array}$ \\
\hline & $\begin{array}{l}\text { 7-membered } \\
\text { endoperoxide } \\
\text { formation }\end{array}$ & A & $\begin{array}{l}1.0 \times 10^{-3}(\mathrm{R}, \mathrm{R}) \\
4.1 \times 10^{-4}(\mathrm{R}, \mathrm{S})\end{array}$ \\
\hline \multirow{7}{*}{$\mathrm{L} 2$} & 1,5 H-shift & $\mathrm{D}$ & $\begin{array}{l}6.6 \times 10^{-2}(\mathrm{R}, \mathrm{R}) \\
1.7 \times 10^{-2}(\mathrm{R}, \mathrm{S})\end{array}$ \\
\hline & 1,5-OH H-shift & $\mathrm{H}-\mathrm{OH}$ & $\begin{array}{l}1.0 \times 10^{-3}(\mathrm{R}, \mathrm{R}) \\
9.4 \times 10^{-4}(\mathrm{R}, \mathrm{S})\end{array}$ \\
\hline & 1,6 H-shift & $\mathrm{E}$ & $\begin{array}{l}3.1 \times 10^{-3}(\mathrm{R}, \mathrm{R}) \\
5.6 \times 10^{-4}(\mathrm{R}, \mathrm{S})\end{array}$ \\
\hline & 1,6 H-shift & $\mathrm{C}$ & $\begin{array}{c}63(\mathrm{R}, \mathrm{R}) \\
130(\mathrm{R}, \mathrm{S}) \\
\end{array}$ \\
\hline & 1,7 H-shift & $\mathrm{F}$ & $\begin{array}{c}0.29(\mathrm{R}, \mathrm{R}) \\
8.1 \times 10^{-2}(\mathrm{R}, \mathrm{S})\end{array}$ \\
\hline & $\begin{array}{l}\text { 7-membered } \\
\text { endoperoxide } \\
\text { formation }\end{array}$ & B & $\begin{array}{l}2.2 \times 10^{-2}(\mathrm{R}, \mathrm{R}) \\
9.7 \times 10^{-2}(\mathrm{R}, \mathrm{S})\end{array}$ \\
\hline & $\begin{array}{l}\text { 8-membered } \\
\text { endoperoxide } \\
\text { formation }\end{array}$ & A & $\begin{array}{l}4.2 \times 10^{-3}(\mathrm{R}, \mathrm{R}) \\
1.5 \times 10^{-3}(\mathrm{R}, \mathrm{S})\end{array}$ \\
\hline L3 & 1,5-OH H-shift & $\mathrm{B}-\mathrm{OH}$ & $4.9 \times 10^{-4}$ \\
\hline $\mathrm{L} 4$ & 1,5-OH H-shift & $\mathrm{B}-\mathrm{OH}$ & $3.6 \times 10^{-6}$ \\
\hline L5 & 1,5 H-shift & $\mathrm{D}$ & $1.7 \times 10^{-3}$ \\
\hline
\end{tabular}




\begin{tabular}{|c|c|c|c|}
\hline & $1,5-\mathrm{OH}$ H-shift & A-OH & $1.1 \times 10^{-3}$ \\
\hline L6 & $1,5-\mathrm{OH}$ H-shift & A-OH & $6.0 \times 10^{-4}$ \\
\hline L7 & $1,5-\mathrm{OH}$ H-shift & B-OH & $6.0 \times 10^{-5}$ \\
\hline L8 & $1,5-\mathrm{OH}$ H-shift & $\mathrm{B}-\mathrm{OH}$ & $9.7 \times 10^{-4}$ \\
\hline L9 & $1,5-\mathrm{OH}$ H-shift & $\mathrm{A}-\mathrm{OH}$ & $1.9 \times 10^{-5}$ \\
\hline L10 & $1,5-\mathrm{OH}$ H-shift & A-OH & $9.4 \times 10^{-4}$ \\
\hline
\end{tabular}


Table S 22. Calculated reaction rate coefficients, $\mathrm{k}$, at $298.15 \mathrm{~K}$ for the unimolecular reactions of the hydroxy peroxy radicals formed from limonene $+\mathrm{OH}+\mathrm{O}_{2}$ (Figure $\mathrm{S} 13$ ). All values are calculated at the B3LYP/6$31+\mathrm{G}(\mathrm{d})$ level of theory with the symmetrical Eckart tunneling approach assuming thermoneutral reactions. The abstraction/addition site refer to the structures in Figure S 12, with "-OH" referring to abstraction of the hydrogen from the hydroxy group on the specified carbon atom. The reactions highlighted in bold are the ones also treated at a higher level of theory.

\begin{tabular}{|c|c|c|c|}
\hline Peroxy radical & Reaction type & $\begin{array}{c}\text { Abstraction/addition } \\
\text { site }\end{array}$ & $\mathrm{k}\left(\mathrm{s}^{-1}\right)$ \\
\hline \multirow{11}{*}{ L1 } & 1,5-OH H-shift & $\mathrm{J}-\mathrm{OH}$ & $\begin{array}{c}0.12(\mathrm{R}, \mathrm{R}) \\
9.5 \times 10^{-2}(\mathrm{R}, \mathrm{S})\end{array}$ \\
\hline & 1,4 H-shift & $\mathrm{J}$ & $\begin{array}{l}1.2 \times 10^{-6}(\mathrm{R}, \mathrm{R}) \\
1.5 \times 10^{-6}(\mathrm{R}, \mathrm{S})\end{array}$ \\
\hline & 1,4 H-shift & $\mathrm{D}$ & $\begin{array}{l}2.0 \times 10^{-6}(\mathrm{R}, \mathrm{R}) \\
2.4 \times 10^{-6}(\mathrm{R}, \mathrm{S})\end{array}$ \\
\hline & 1,4 H-shift & I & $\begin{array}{l}6.2 \times 10^{-10}(\mathrm{R}, \mathrm{R}) \\
9.6 \times 10^{-10}(\mathrm{R}, \mathrm{S})\end{array}$ \\
\hline & 1,5 H-shift & $\mathrm{E}$ & $\begin{array}{l}1.3 \times 10^{-5}(\mathrm{R}, \mathrm{R}) \\
3.9 \times 10^{-5}(\mathrm{R}, \mathrm{S})\end{array}$ \\
\hline & 1,5 H-shift & $\mathbf{C}$ & $\begin{array}{l}25(R, R) \\
6.8(R, S)\end{array}$ \\
\hline & 1,6 H-shift & $\mathbf{F}$ & $\begin{array}{c}0.64(\mathrm{R}, \mathrm{R}) \\
1.9(\mathrm{R}, \mathrm{S})\end{array}$ \\
\hline & 1,6 H-shift & B & $\begin{array}{l}1.5 \times 10^{-17}(\mathrm{R}, \mathrm{R}) \\
6.2 \times 10^{-18}(\mathrm{R}, \mathrm{S})\end{array}$ \\
\hline & $1,8 \mathrm{H}$-shift & G & $\begin{array}{l}3.7 \times 10^{-9}(\mathrm{R}, \mathrm{R}) \\
2.7 \times 10^{-9}(\mathrm{R}, \mathrm{S})\end{array}$ \\
\hline & $\begin{array}{l}\text { 6-membered } \\
\text { endoperoxide } \\
\text { formation }\end{array}$ & B & $\begin{array}{l}19(\mathrm{R}, \mathrm{R}) \\
3.4(\mathrm{R}, \mathrm{S})\end{array}$ \\
\hline & $\begin{array}{l}\text { 7-membered } \\
\text { endoperoxide } \\
\text { formation }\end{array}$ & $\mathbf{A}$ & $\begin{array}{l}2.3 \times 10^{-2}(\mathbf{R}, \mathbf{R}) \\
8.4 \times 10^{-3}(\mathbf{R}, \mathrm{S})\end{array}$ \\
\hline \multirow{4}{*}{ L2 } & 1,5-OH H-shift & H-OH & $\begin{array}{l}0.80(R, R) \\
0.78(R, S)\end{array}$ \\
\hline & 1,5 H-shift & D & $\begin{array}{c}0.15(R, R) \\
4.4 \times 10^{-2}(R, S) \\
\end{array}$ \\
\hline & 1,6 H-shift & $\mathbf{E}$ & $\begin{array}{l}5.7 \times 10^{-3}(\mathrm{R}, \mathrm{R}) \\
1.3 \times 10^{-3}(\mathrm{R}, \mathrm{S})\end{array}$ \\
\hline & 1,6 H-shift & $\mathrm{C}$ & $\begin{array}{l}3.3 \times 10^{2}(\mathrm{R}, \mathrm{R}) \\
1.0 \times 10^{3}(\mathrm{R}, \mathrm{S})\end{array}$ \\
\hline
\end{tabular}




\begin{tabular}{|c|c|c|c|}
\hline & 1,7 H-shift & $\mathbf{F}$ & $\begin{array}{l}0.58(R, R) \\
0.16(R, S)\end{array}$ \\
\hline & 1,9 H-shift & $\mathrm{G}$ & $\begin{array}{l}1.3 \times 10^{-5}(\mathrm{R}, \mathrm{R}) \\
1.3 \times 10^{-5}(\mathrm{R}, \mathrm{S})\end{array}$ \\
\hline & $\begin{array}{c}\text { 7-membered } \\
\text { endoperoxide } \\
\text { formation }\end{array}$ & B & $\begin{array}{l}0.24(R, R) \\
0.94(R, S)\end{array}$ \\
\hline & $\begin{array}{c}\text { 8-membered } \\
\text { endoperoxide } \\
\text { formation } \\
\end{array}$ & $\mathbf{A}$ & $\begin{array}{l}4.9 \times 10^{-2}(R, R) \\
1.9 \times 10^{-2}(R, S)\end{array}$ \\
\hline \multirow{4}{*}{ L3 } & 1,5 H-shift & $E$ & $1.9 \times 10^{-7}$ \\
\hline & 1,5-OH H-shift & B-OH & 0.75 \\
\hline & 1,5 H-shift & $\mathrm{C}$ & $3.2 \times 10^{-7}$ \\
\hline & 1,6 H-shift & $\mathrm{D}$ & $1.4 \times 10^{-4}$ \\
\hline \multirow{6}{*}{ L4 } & 1,5-OH H-shift & B-OH & $5.3 \times 10^{-4}$ \\
\hline & 1,4 H-shift & $\mathrm{B}$ & $5.4 \times 10^{-5}$ \\
\hline & 1,5 H-shift & $E$ & $1.2 \times 10^{-6}$ \\
\hline & 1,5 H-shift & $\mathrm{C}$ & $1.4 \times 10^{-7}$ \\
\hline & 1,8 H-shift & $\mathrm{I}$ & $2.3 \times 10^{-8}$ \\
\hline & $\begin{array}{c}\text { 8-membered } \\
\text { endoperoxide } \\
\text { formation }\end{array}$ & $\mathrm{J}$ & $2.2 \times 10^{-7}$ \\
\hline \multirow{4}{*}{ L5 } & 1,5 H-shift & $\mathrm{F}$ & $6.8 \times 10^{-7}$ \\
\hline & 1,5-OH H-shift & A-OH & 1.6 \\
\hline & 1,5 H-shift & D & $8.8 \times 10^{-3}$ \\
\hline & 1,6 H-shift & $E$ & $1.0 \times 10^{-9}$ \\
\hline \multirow{5}{*}{ L6 } & 1,5-OH H-shift & A-OH & 0.70 \\
\hline & 1,5 H-shift & $\mathrm{F}$ & $5.8 \times 10^{-10}$ \\
\hline & 1,6 H-shift & $E$ & $9.8 \times 10^{-10}$ \\
\hline & 1,7 H-shift & I & $5.1 \times 10^{-6}$ \\
\hline & $\begin{array}{l}\text { 7-membered } \\
\text { endoperoxide } \\
\text { formation }\end{array}$ & $\mathrm{J}$ & $4.0 \times 10^{-4}$ \\
\hline \multirow{5}{*}{ L7 } & 1,5-OH H-shift & B-OH & $5.4 \times 10^{-2}$ \\
\hline & 1,4 H-shift & $\mathrm{B}$ & $2.8 \times 10^{-5}$ \\
\hline & 1,5 H-shift & $\mathrm{E}$ & $5.2 \times 10^{-9}$ \\
\hline & 1,5 H-shift & $\mathrm{C}$ & $4.4 \times 10^{-10}$ \\
\hline & 1,6 H-shift & $\mathrm{D}$ & $1.3 \times 10^{-5}$ \\
\hline \multirow{3}{*}{ L8 } & $1,5 \mathrm{H}$-shift & $E$ & $2.6 \times 10^{-6}$ \\
\hline & 1,5-OH H-shift & B-OH & 0.96 \\
\hline & $1,5 \mathrm{H}$-shift & $\mathrm{C}$ & $2.1 \times 10^{-6}$ \\
\hline
\end{tabular}




\begin{tabular}{|c|c|c|c|}
\hline & 1,8 H-shift & I & $2.7 \times 10^{-8}$ \\
\hline & $\begin{array}{c}\text { 8-membered } \\
\text { endoperoxide } \\
\text { formation }\end{array}$ & $\mathrm{J}$ & $2.9 \times 10^{-7}$ \\
\hline \multirow{4}{*}{ L9 } & 1,5 H-shift & $\mathrm{F}$ & $1.0 \times 10^{-7}$ \\
\hline & 1,5-OH H-shift & A-OH & $2.4 \times 10^{-3}$ \\
\hline & 1,5 H-shift & $\mathrm{D}$ & $2.7 \times 10^{-6}$ \\
\hline & 1,6 H-shift & $\mathrm{E}$ & $1.2 \times 10^{-10}$ \\
\hline \multirow{5}{*}{ L10 } & 1,5 H-shift & $\mathrm{F}$ & $1.4 \times 10^{-10}$ \\
\hline & 1,5-OH H-shift & $\mathrm{A}-\mathrm{OH}$ & 0.12 \\
\hline & 1,6 H-shift & $\mathrm{E}$ & $1.2 \times 10^{-10}$ \\
\hline & 1,7 H-shift & I & $8.5 \times 10^{-7}$ \\
\hline & $\begin{array}{l}\text { 7-membered } \\
\text { endoperoxide } \\
\text { formation }\end{array}$ & $\mathrm{J}$ & $2.5 \times 10^{-7}$ \\
\hline
\end{tabular}


Table S 23. CCSD(T)-F12a/VDZ-F12 (abbreviated F12) and $\omega$ B97X-D/aug-cc-pVTZ barrier heights ( $\mathrm{E}_{0}$, in $\mathrm{kcal} / \mathrm{mol})$, summed partition function ratios $\left(\mathrm{Q}_{\mathrm{TS}} / \mathrm{Q}_{\mathrm{R}}\right)$ and Eckart tunneling coefficients $(\kappa)$ used for calculating the corresponding MC-TST rate coefficients in the preceding tables for limonene.

\begin{tabular}{|c|c|c|c|c|c|c|c|c|}
\hline $\begin{array}{l}\text { Peroxy } \\
\text { radical }\end{array}$ & $\begin{array}{c}\text { Reaction } \\
\text { type }\end{array}$ & $\begin{array}{l}\text { Abstraction/ } \\
\text { addition site }\end{array}$ & \begin{tabular}{|c|}
$\mathrm{R} / \mathrm{S}$ \\
Isomer \\
\end{tabular} & $\begin{array}{c}\mathrm{E}_{0} \\
(\mathrm{~F} 12) \\
\end{array}$ & $\begin{array}{c}\kappa \\
(\mathrm{F} 12) \\
\end{array}$ & $\begin{array}{c}\mathrm{E}_{0} \\
(\omega \mathrm{B} 97 \mathrm{X}-\mathrm{D})\end{array}$ & $\mathrm{Q}_{\mathrm{TS}} / \mathrm{Q}_{\mathrm{R}}$ & $\begin{array}{c}\kappa \\
(\omega \mathrm{B} 97 \mathrm{X}-\mathrm{D})\end{array}$ \\
\hline \multirow{10}{*}{ L1 } & \multirow{2}{*}{$\begin{array}{c}\text { 1,5-OH H- } \\
\text { shift }\end{array}$} & \multirow{2}{*}{$\mathrm{J}-\mathrm{OH}$} & $\mathrm{R}, \mathrm{R}$ & - & - & 21.3 & $1.58 \times 10^{-1}$ & 1.0 \\
\hline & & & $\mathrm{R}, \mathrm{S}$ & - & - & 22.1 & $2.93 \times 10^{-1}$ & 0.7 \\
\hline & \multirow{2}{*}{$1,5 \mathrm{H}$-shift } & \multirow{2}{*}{$\mathrm{C}$} & $\mathrm{R}, \mathrm{R}$ & 18.6 & 238.1 & 19.0 & $1.08 \times 10^{-1}$ & 214.0 \\
\hline & & & $\mathrm{R}, \mathrm{S}$ & 20.5 & 207.8 & 20.4 & $4.14 \times 10^{-1}$ & 180.3 \\
\hline & \multirow{2}{*}{ 1,6 H-shift } & \multirow{2}{*}{$\mathrm{F}$} & $\mathrm{R}, \mathrm{R}$ & 20.2 & 165.8 & 20.4 & $1.32 \times 10^{-1}$ & 141.1 \\
\hline & & & $\mathrm{R}, \mathrm{S}$ & 19.2 & 118.8 & 19.7 & $1.07 \times 10^{-1}$ & 106.0 \\
\hline & \multirow{2}{*}{$\begin{array}{c}\text { 6-membered } \\
\text { endoperoxide } \\
\text { formation }\end{array}$} & \multirow{2}{*}{ B } & $\mathrm{R}, \mathrm{R}$ & 15.0 & 1.4 & 16.0 & $4.75 \times 10^{-2}$ & 1.4 \\
\hline & & & $\mathrm{R}, \mathrm{S}$ & 16.6 & 1.4 & 17.4 & $1.53 \times 10^{-1}$ & 1.4 \\
\hline & \multirow{2}{*}{$\begin{array}{c}\text { 7-membered } \\
\text { endoperoxide } \\
\text { formation }\end{array}$} & \multirow{2}{*}{ A } & $\mathrm{R}, \mathrm{R}$ & 17.8 & 1.3 & 19.9 & $4.92 \times 10^{-2}$ & 1.3 \\
\hline & & & $\mathrm{R}, \mathrm{S}$ & 18.9 & 1.3 & 20.9 & $1.01 \times 10^{-1}$ & 1.3 \\
\hline \multirow{7}{*}{ L2 } & \multirow{2}{*}{ 1,5 H-shift } & \multirow{2}{*}{ D } & $\mathrm{R}, \mathrm{R}$ & 18.4 & 42.3 & 19.2 & $3.82 \times 10^{-2}$ & 34.3 \\
\hline & & & $\mathrm{R}, \mathrm{S}$ & 19.9 & 47.5 & 20.6 & $8.81 \times 10^{-2}$ & 38.1 \\
\hline & \multirow{2}{*}{$\begin{array}{l}\text { 1,5-OH H- } \\
\text { shift }\end{array}$} & \multirow{2}{*}{$\mathrm{H}-\mathrm{OH}$} & $\mathrm{R}, \mathrm{R}$ & - & - & 20.6 & $2.26 \times 10^{-1}$ & 1.1 \\
\hline & & & $\mathrm{R}, \mathrm{S}$ & - & - & 20.9 & $2.97 \times 10^{-1}$ & 0.9 \\
\hline & \multirow{2}{*}{ 1,6 H-shift } & \multirow{2}{*}{$\mathrm{E}$} & $\mathrm{R}, \mathrm{R}$ & 20.64 & 48.0 & 20.8 & $3.15 \times 10^{-2}$ & 29.6 \\
\hline & & & $\mathrm{R}, \mathrm{S}$ & 22.9 & 61.5 & 23.0 & $1.80 \times 10^{-1}$ & 35.7 \\
\hline & 1,6 H-shift & $\mathrm{C}$ & $\mathrm{R}, \mathrm{R}$ & 16.9 & 230.4 & 16.3 & $5.40 \times 10^{-2}$ & 179.5 \\
\hline
\end{tabular}




\begin{tabular}{|c|c|c|c|c|c|c|c|c|}
\hline & & & $\mathrm{R}, \mathrm{S}$ & 16.4 & 127.9 & 15.5 & $4.70 \times 10^{-2}$ & 101.5 \\
\hline & \multirow{2}{*}{ 1,7 H-shift } & \multirow{2}{*}{$\mathrm{F}$} & $\mathrm{R}, \mathrm{R}$ & 18.1 & 59.3 & 17.7 & $8.17 \times 10^{-3}$ & 50.6 \\
\hline & & & $\mathrm{R}, \mathrm{S}$ & 20.7 & 188.4 & 20.6 & $9.91 \times 10^{-2}$ & 152.4 \\
\hline & \multirow{2}{*}{$\begin{array}{l}\text { 7-membered } \\
\text { endoperoxide } \\
\text { formation }\end{array}$} & \multirow{2}{*}{ B } & $\mathrm{R}, \mathrm{R}$ & 18.2 & 1.4 & 18.1 & $4.94 \times 10^{-2}$ & 1.4 \\
\hline & & & $\mathrm{R}, \mathrm{S}$ & 17.1 & 1.4 & 16.9 & $2.59 \times 10^{-2}$ & 1.4 \\
\hline & \multirow{2}{*}{$\begin{array}{l}\text { 8-membered } \\
\text { endoperoxide } \\
\text { formation }\end{array}$} & \multirow{2}{*}{ A } & $\mathrm{R}, \mathrm{R}$ & 16.3 & 1.3 & 18.5 & $1.74 \times 10^{-2}$ & 1.3 \\
\hline & & & $\mathrm{R}, \mathrm{S}$ & 17.6 & 1.3 & 19.5 & $3.78 \times 10^{-2}$ & 1.3 \\
\hline $\mathrm{L} 3$ & $\begin{array}{c}\text { 1,5-OH H- } \\
\text { shift }\end{array}$ & $\mathrm{B}-\mathrm{OH}$ & - & - & - & 20.7 & $1.86 \times 10^{-1}$ & 0.7 \\
\hline \multirow{2}{*}{ L5 } & 1,5 H-shift & $\mathrm{D}$ & - & 21.8 & 292.0 & 22.8 & $4.44 \times 10^{-2}$ & 296.7 \\
\hline & $\begin{array}{c}\text { 1,5-OH H- } \\
\text { shift }\end{array}$ & $\mathrm{B}-\mathrm{OH}$ & - & - & - & 19.8 & $1.04 \times 10^{-1}$ & 0.5 \\
\hline L8 & $\begin{array}{c}\text { 1,5-OH H- } \\
\text { shift }\end{array}$ & $\mathrm{B}-\mathrm{OH}$ & - & - & - & 20.4 & $1.68 \times 10^{-1}$ & 0.9 \\
\hline L9 & $\begin{array}{c}\text { 1,5-OH H- } \\
\text { shift }\end{array}$ & $\mathrm{A}-\mathrm{OH}$ & - & - & - & 21.7 & $1.62 \times 10^{-2}$ & 1.5 \\
\hline L10 & $\begin{array}{c}\text { 1,5-OH H- } \\
\text { shift }\end{array}$ & $\mathrm{A}-\mathrm{OH}$ & - & - & - & 21.1 & $3.38 \times 10^{-1}$ & 1.2 \\
\hline
\end{tabular}


Table S 24. B3LYP/6-31+G(d) barrier heights $\left(\mathrm{E}_{0}\right.$, in kcal/mol), summed partition function ratios $\left(\mathrm{Q}_{\mathrm{TS}} / \mathrm{Q}_{\mathrm{R}}\right)$ and Eckart tunneling coefficients $(\kappa)$ used for calculating the corresponding MC-TST rate coefficients in the preceding tables for limonene.

\begin{tabular}{|c|c|c|c|c|c|c|}
\hline $\begin{array}{l}\text { Peroxy } \\
\text { radical }\end{array}$ & Reaction type & $\begin{array}{l}\text { Abstraction/ } \\
\text { addition site }\end{array}$ & $\begin{array}{c}\mathrm{R} / \mathrm{S} \\
\text { Isomer }\end{array}$ & $\begin{array}{c}\mathrm{E}_{0} \\
(\mathrm{~B} 3 \mathrm{LYP})\end{array}$ & $\mathrm{Q}_{\mathrm{TS}} / \mathrm{Q}_{\mathrm{R}}$ & $\begin{array}{c}\kappa \\
\text { (B3LYP) }\end{array}$ \\
\hline \multirow{17}{*}{ L1 } & \multirow{2}{*}{ 1,5-OH H-shift } & \multirow{2}{*}{$\mathrm{J}-\mathrm{OH}$} & $\mathrm{R}, \mathrm{R}$ & 18.6 & $1.75 \times 10^{-1}$ & 4.4 \\
\hline & & & $\mathrm{R}, \mathrm{S}$ & 19.1 & $3.04 \times 10^{-1}$ & 5.3 \\
\hline & \multirow{2}{*}{ 1,4 H-shift } & \multirow{2}{*}{$\mathrm{J}$} & $\mathrm{R}, \mathrm{R}$ & 29.2 & $2.05 \times 10^{-1}$ & 2574.3 \\
\hline & & & $\mathrm{R}, \mathrm{S}$ & 30.0 & $8.39 \times 10^{-1}$ & 2911.9 \\
\hline & \multirow{2}{*}{ 1,4 H-shift } & \multirow{2}{*}{$\mathrm{D}$} & $\mathrm{R}, \mathrm{R}$ & 28.9 & $1.53 \times 10^{-1}$ & 3261.2 \\
\hline & & & $\mathrm{R}, \mathrm{S}$ & 29.3 & $3.27 \times 10^{-1}$ & 3537.9 \\
\hline & \multirow{2}{*}{ 1,4 H-shift } & \multirow{2}{*}{ I } & $\mathrm{R}, \mathrm{R}$ & 37.2 & $5.47 \times 10^{-1}$ & 359100.1 \\
\hline & & & $\mathrm{R}, \mathrm{S}$ & 37.3 & $7.26 \times 10^{-1}$ & 460692.2 \\
\hline & \multirow{2}{*}{ 1,5 H-shift } & \multirow{2}{*}{$\mathrm{E}$} & $\mathrm{R}, \mathrm{R}$ & 26.0 & $2.05 \times 10^{-1}$ & 128.5 \\
\hline & & & $\mathrm{R}, \mathrm{S}$ & 25.8 & $5.63 \times 10^{-1}$ & 94.7 \\
\hline & \multirow{2}{*}{ 1,5 H-shift } & \multirow{2}{*}{$\mathrm{C}$} & $\mathrm{R}, \mathrm{R}$ & 16.7 & $8.79 \times 10^{-2}$ & 80.0 \\
\hline & & & $\mathrm{R}, \mathrm{S}$ & 18.5 & $3.12 \times 10^{-1}$ & 137.0 \\
\hline & \multirow{2}{*}{ 1,6 H-shift } & \multirow{2}{*}{$\mathrm{F}$} & $\mathrm{R}, \mathrm{R}$ & 19.1 & $1.00 \times 10^{-1}$ & 108.4 \\
\hline & & & $\mathrm{R}, \mathrm{S}$ & 18.2 & $8.45 \times 10^{-2}$ & 78.4 \\
\hline & \multirow{2}{*}{ 1,6 H-shift } & \multirow{2}{*}{ B } & $\mathrm{R}, \mathrm{R}$ & 45.2 & $6.54 \times 10^{-2}$ & 46958.0 \\
\hline & & & $\mathrm{R}, \mathrm{S}$ & 46.4 & $1.25 \times 10^{-1}$ & 87165.0 \\
\hline & 1,8 H-shift & $\mathrm{G}$ & $\mathrm{R}, \mathrm{R}$ & 30.8 & $2.62 \times 10^{-2}$ & 849.5 \\
\hline
\end{tabular}




\begin{tabular}{|c|c|c|c|c|c|c|}
\hline & & & $\mathrm{R}, \mathrm{S}$ & 30.8 & $2.77 \times 10^{-2}$ & 591.0 \\
\hline & \multirow{2}{*}{$\begin{array}{l}\text { 6-membered } \\
\text { endoperoxide } \\
\text { formation }\end{array}$} & \multirow{2}{*}{ B } & $\mathrm{R}, \mathrm{R}$ & 13.9 & $3.61 \times 10^{-2}$ & 1.2 \\
\hline & & & $\mathrm{R}, \mathrm{S}$ & 15.4 & $8.33 \times 10^{-2}$ & 1.3 \\
\hline & \multirow{2}{*}{$\begin{array}{l}\text { 7-membered } \\
\text { endoperoxide } \\
\text { formation }\end{array}$} & \multirow{2}{*}{ A } & $\mathrm{R}, \mathrm{R}$ & 17.8 & $3.62 \times 10^{-2}$ & 1.2 \\
\hline & & & $\mathrm{R}, \mathrm{S}$ & 18.8 & $6.39 \times 10^{-2}$ & 1.2 \\
\hline \multirow{15}{*}{ L2 } & \multirow{2}{*}{ 1,5-OH H-shift } & \multirow{2}{*}{$\mathrm{H}-\mathrm{OH}$} & $\mathrm{R}, \mathrm{R}$ & 17.1 & $9.54 \times 10^{-2}$ & 4.5 \\
\hline & & & $\mathrm{R}, \mathrm{S}$ & 17.4 & $1.66 \times 10^{-1}$ & 4.5 \\
\hline & & & $\mathrm{R}, \mathrm{R}$ & 18.9 & $2.47 \times 10^{-2}$ & 77.7 \\
\hline & - & 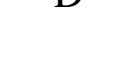 & $\mathrm{R}, \mathrm{S}$ & 20.0 & $3.83 \times 10^{-2}$ & 91.0 \\
\hline & & & $\mathrm{R}, \mathrm{R}$ & 20.9 & $1.72 \times 10^{-2}$ & 109.2 \\
\hline & ч & $\mathrm{L}$ & $\mathrm{R}, \mathrm{S}$ & 22.8 & $6.69 \times 10^{-2}$ & 161.1 \\
\hline & & & $\mathrm{R}, \mathrm{R}$ & 14.2 & $2.40 \times 10^{-2}$ & 55.1 \\
\hline & 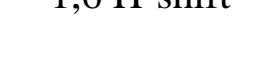 & 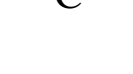 & $\mathrm{R}, \mathrm{S}$ & 13.2 & $2.20 \times 10^{-2}$ & 32.9 \\
\hline & & & $\mathrm{R}, \mathrm{R}$ & 17.1 & $6.57 \times 10^{-3}$ & 47.6 \\
\hline & 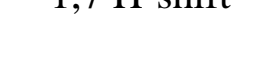 & 1 & $\mathrm{R}, \mathrm{S}$ & 19.1 & $3.04 \times 10^{-2}$ & 86.9 \\
\hline & & & $\mathrm{R}, \mathrm{R}$ & 24.6 & $9.76 \times 10^{-3}$ & 229.5 \\
\hline & 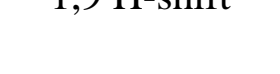 & 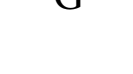 & $\mathrm{R}, \mathrm{S}$ & 24.6 & $1.06 \times 10^{-2}$ & 216.1 \\
\hline & 7-membered & & $\mathrm{R}, \mathrm{R}$ & 16.2 & $2.42 \times 10^{-2}$ & 1.2 \\
\hline & formation & D & $\mathrm{R}, \mathrm{S}$ & 15.1 & $1.49 \times 10^{-2}$ & 1.2 \\
\hline & $\begin{array}{l}\text { 8-membered } \\
\text { endoperoxide }\end{array}$ & A & $\mathrm{R}, \mathrm{R}$ & 16.6 & $9.81 \times 10^{-3}$ & 1.2 \\
\hline
\end{tabular}




\begin{tabular}{|c|c|c|c|c|c|c|}
\hline & formation & & $\mathrm{R}, \mathrm{S}$ & 17.5 & $1.77 \times 10^{-2}$ & 1.2 \\
\hline \multirow{4}{*}{ L3 } & 1,5 H-shift & $\mathrm{E}$ & - & 28.2 & $5.36 \times 10^{-2}$ & 295.4 \\
\hline & 1,5-OH H-shift & $\mathrm{B}-\mathrm{OH}$ & - & 17.5 & $1.40 \times 10^{-1}$ & 5.7 \\
\hline & 1,5 H-shift & $\mathrm{C}$ & - & 28.2 & $9.55 \times 10^{-2}$ & 247.0 \\
\hline & 1,6 H-shift & $\mathrm{D}$ & - & 24.0 & $3.74 \times 10^{-2}$ & 227.3 \\
\hline \multirow{6}{*}{$\mathrm{L} 4$} & 1,5-OH H-shift & $\mathrm{B}-\mathrm{OH}$ & - & & & \\
\hline & 1,4 H-shift & B & - & 26.0 & $1.65 \times 10^{-1}$ & 560.2 \\
\hline & 1,5 H-shift & $\mathrm{E}$ & - & 27.4 & $9.52 \times 10^{-2}$ & 259.4 \\
\hline & 1,5 H-shift & $\mathrm{C}$ & - & 29.3 & $9.41 \times 10^{-2}$ & 751.5 \\
\hline & 1,8 H-shift & I & - & 29.3 & $1.11 \times 10^{-2}$ & 955.9 \\
\hline & $\begin{array}{l}\text { 8-membered } \\
\text { endoperoxide } \\
\text { formation }\end{array}$ & $\mathrm{J}$ & - & 24.4 & $2.07 \times 10^{-2}$ & 1.3 \\
\hline \multirow{4}{*}{ L5 } & 1,5 H-shift & $\mathrm{F}$ & - & 27.8 & $9.63 \times 10^{-2}$ & 292.1 \\
\hline & 1,5-OH H-shift & $\mathrm{A}-\mathrm{OH}$ & - & 16.8 & $7.85 \times 10^{-2}$ & 6.6 \\
\hline & 1,5 H-shift & $\mathrm{D}$ & - & 21.2 & $3.78 \times 10^{-2}$ & 124.9 \\
\hline & 1,6 H-shift & $\mathrm{E}$ & - & 32.1 & $8.08 \times 10^{-2}$ & 715.8 \\
\hline \multirow{5}{*}{ L6 } & 1,5-OH H-shift & $\mathrm{A}-\mathrm{OH}$ & - & & & \\
\hline & 1,5 H-shift & $\mathrm{F}$ & - & 33.4 & $1.68 \times 10^{-1}$ & 1819.1 \\
\hline & 1,6 H-shift & $E$ & - & 32.9 & $1.47 \times 10^{-1}$ & 1291.5 \\
\hline & 1,7 H-shift & I & - & 26.4 & $1.78 \times 10^{-2}$ & 1008.9 \\
\hline & $\begin{array}{l}\text { 7-membered } \\
\text { endoperoxide } \\
\text { formation }\end{array}$ & $\mathrm{J}$ & - & 19.4 & $8.75 \times 10^{-3}$ & 1.3 \\
\hline \multirow{5}{*}{ L7 } & 1,5-OH H-shift & $\mathrm{B}-\mathrm{OH}$ & - & & & \\
\hline & 1,4 H-shift & $\mathrm{B}$ & - & 26.9 & $2.56 \times 10^{-1}$ & 832.8 \\
\hline & 1,5 H-shift & $\mathrm{E}$ & - & 31.8 & $3.05 \times 10^{-1}$ & 511.3 \\
\hline & 1,5 H-shift & $\mathrm{C}$ & - & 33.4 & $1.38 \times 10^{-1}$ & 1669.0 \\
\hline & 1,6 H-shift & $\mathrm{D}$ & - & 27.0 & $1.55 \times 10^{-1}$ & 824.6 \\
\hline \multirow{5}{*}{ L8 } & 1,5 H-shift & $\mathrm{E}$ & - & 27.2 & $1.30 \times 10^{-1}$ & 285.2 \\
\hline & 1,5-OH H-shift & $\mathrm{B}-\mathrm{OH}$ & - & 17.5 & $1.72 \times 10^{-1}$ & 6.1 \\
\hline & 1,5 H-shift & $\mathrm{C}$ & - & 27.1 & $1.18 \times 10^{-1}$ & 208.7 \\
\hline & 1,8 H-shift & $\mathrm{I}$ & - & 29.2 & $1.14 \times 10^{-2}$ & 884.2 \\
\hline & $\begin{array}{l}\text { 8-membered } \\
\text { endoperoxide } \\
\text { formation }\end{array}$ & $\mathrm{J}$ & - & 24.3 & $2.40 \times 10^{-2}$ & 1.3 \\
\hline L9 & 1,5 H-shift & $\mathrm{F}$ & - & 29.7 & $1.07 \times 10^{-1}$ & 917.0 \\
\hline
\end{tabular}




\begin{tabular}{|c|c|c|c|c|c|c|}
\hline \multirow{7}{*}{} & $1,5-\mathrm{OH}$ H-shift & A-OH & - & 20.3 & $3.20 \times 10^{-2}$ & 9.3 \\
\cline { 2 - 7 } & 1,5 H-shift & $\mathrm{D}$ & - & 26.1 & $4.75 \times 10^{-2}$ & 133.2 \\
\cline { 2 - 7 } & $1,6 \mathrm{H}$-shift & $\mathrm{E}$ & - & 34.3 & $8.15 \times 10^{-2}$ & 3155.2 \\
\hline \multirow{6}{*}{ L10 } & $1,5 \mathrm{H}$-shift & $\mathrm{F}$ & - & 33.0 & $6.59 \times 10^{-2}$ & 509.1 \\
\cline { 2 - 7 } & $1,5-\mathrm{OH}$ H-shift & $\mathrm{A}-\mathrm{OH}$ & - & 18.3 & $8.20 \times 10^{-2}$ & 6.8 \\
\cline { 2 - 7 } & $1,6 \mathrm{H}$-shift & $\mathrm{E}$ & & 33.3 & $3.63 \times 10^{-2}$ & 1264.0 \\
\cline { 2 - 7 } & 1,7 H-shift & $\mathrm{I}$ & & 27.1 & $9.41 \times 10^{-3}$ & 1026.9 \\
\cline { 2 - 7 } & $\begin{array}{c}7-\text { membered } \\
\text { endoperoxide } \\
\text { formation }\end{array}$ & $\mathrm{J}$ & & 23.7 & $6.77 \times 10^{-3}$ & 1.3 \\
\hline
\end{tabular}




\section{S3.6 Terpinolene}<smiles>CC1=CCC(=C(I)I)CC1</smiles>

Figure S 14. Structure of terpinolene with atom labeling of the carbon atoms used to define the unimolecular reactions.

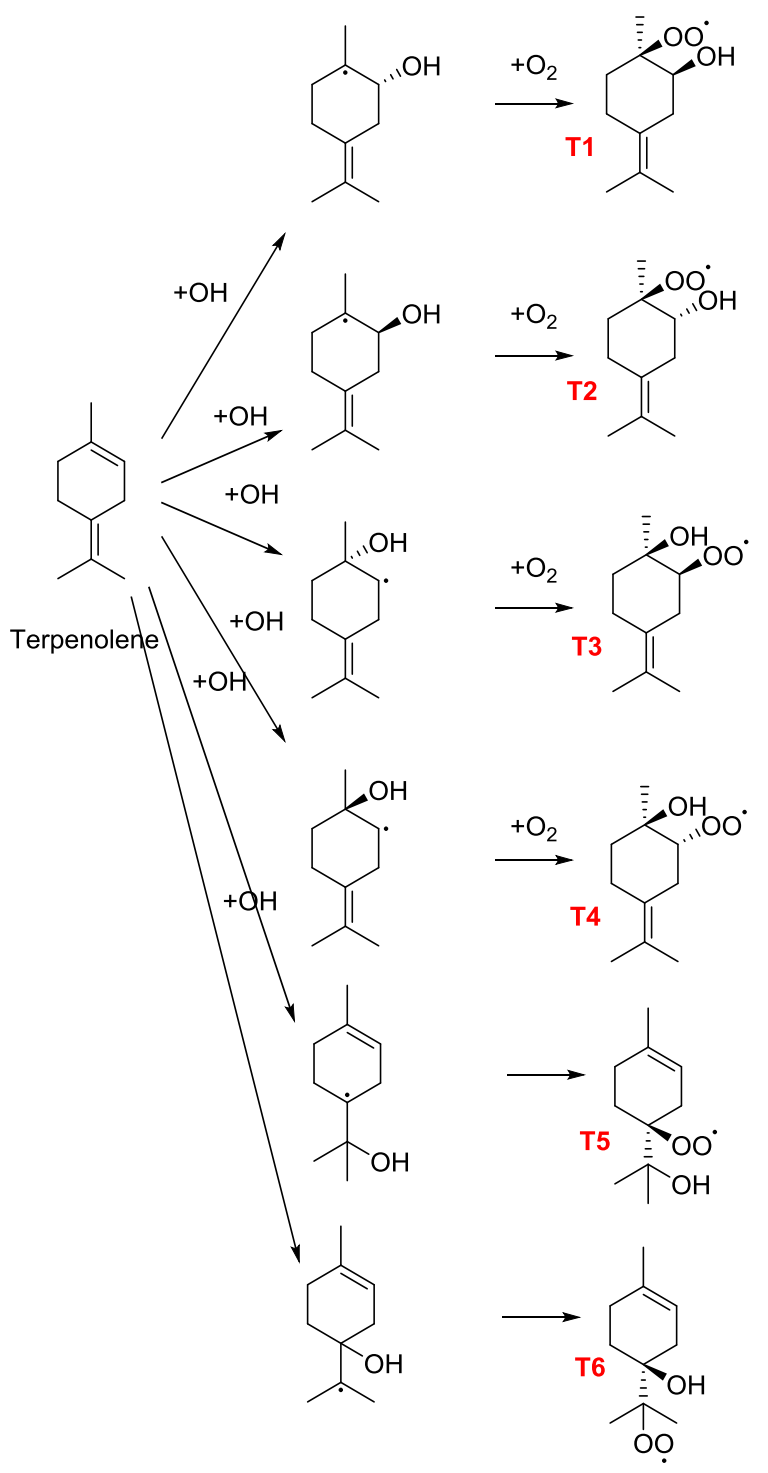

Figure S 15. Formation of the peroxy different hydroxy peroxy radicals from terpinolene $+\mathrm{OH}+\mathrm{O}_{2}$. The seemingly missing conformers are mirror images of the ones present due to the symmetry in the molecule. 
Table S 25. Calculated MC-TST reaction rate coefficients, $\mathrm{k}$, at $298.15 \mathrm{~K}$ for the unimolecular reactions of the hydroxy peroxy radicals formed from terpinolene $+\mathrm{OH}+\mathrm{O}_{2}$ (Figure $\mathrm{S}$ 15). The rate coefficients are calculated using the approach by Møller et al. ${ }^{1}$ The electronic energies of the lowest-energy conformers and IRC end-points are calculated at the CCSD(T)-F12a/VDZ-F12 level while the remaining parameters are calculated at the $\omega$ B97X-D/aug-cc-pVTZ level. Tunneling is based on IRC end-points. The abstraction/addition site refer to the structures in Figure $\mathrm{S} \mathrm{14}$, with "-OH" referring to abstraction of the hydrogen from the hydroxy group on the specified carbon atom.

\begin{tabular}{|c|c|c|c|}
\hline Peroxy Radical & Reaction type & $\begin{array}{c}\text { Abstraction/addition } \\
\text { site }\end{array}$ & $\mathrm{k}\left(\mathrm{s}^{-1}\right)$ \\
\hline \multirow{4}{*}{$\mathrm{T} 1$} & 1,5 H-shift & $\mathrm{E}$ & $1.2 \times 10^{-2}$ \\
\hline & 1,5-OH H-shift & $\mathrm{B}-\mathrm{OH}$ & - \\
\hline & 1,5 H-shift & $\mathrm{C}$ & $1.0 \times 10^{-2}$ \\
\hline & $\begin{array}{l}\text { 6-membered } \\
\text { endoperoxide } \\
\text { formation }\end{array}$ & $\mathrm{D}$ & $5.4 \times 10^{-2}$ \\
\hline \multirow[b]{2}{*}{$\mathrm{T} 2$} & 1,5-OH H-shift & $\mathrm{B}-\mathrm{OH}$ & - \\
\hline & $\begin{array}{l}\text { 6-membered } \\
\text { endoperoxide } \\
\text { formation }\end{array}$ & $\mathrm{D}$ & $9.8 \times 10^{-4}$ \\
\hline \multirow[b]{2}{*}{$\mathrm{T} 3$} & 1,5-OH H-shift & $\mathrm{A}-\mathrm{OH}$ & - \\
\hline & $\begin{array}{l}\text { 5-membered } \\
\text { endoperoxide } \\
\text { formation }\end{array}$ & $\mathrm{D}$ & 1.4 \\
\hline \multirow{4}{*}{$\mathrm{T} 4$} & 1,5-OH H-shift & $\mathrm{A}-\mathrm{OH}$ & - \\
\hline & 1,7 H-shift & $\mathrm{J}$ & 0.92 \\
\hline & $\begin{array}{c}\text { 5-membered } \\
\text { endoperoxide } \\
\text { formation }\end{array}$ & $\mathrm{D}$ & 6.3 \\
\hline & $\begin{array}{l}\text { 6-membered } \\
\text { endoperoxide } \\
\text { formation }\end{array}$ & $\mathrm{H}$ & 0.73 \\
\hline \multirow{4}{*}{$\mathrm{T} 5$} & 1,4 H-shift & $\mathrm{C}$ & $5.5 \times 10^{-3}$ \\
\hline & 1,5 H-shift & $\mathrm{F}$ & 0.13 \\
\hline & 1,5-OH H-shift & $\mathrm{H}-\mathrm{OH}$ & - \\
\hline & $\begin{array}{c}\text { 5-membered } \\
\text { endoperoxide } \\
\text { formation }\end{array}$ & B & $6.6 \times 10^{-2}$ \\
\hline \multirow{2}{*}{ T6 } & 1,5-OH H-shift & $\mathrm{D}-\mathrm{OH}$ & - \\
\hline & 1,5 H-shift & $\mathrm{C}$ & 4.1 \\
\hline
\end{tabular}




\begin{tabular}{|c|c|c|c|}
\hline & 1,6 H-shift & F & 0.57 \\
\cline { 2 - 4 } & $\begin{array}{c}\text { 6-membered } \\
\text { endoperoxide } \\
\text { formation }\end{array}$ & B & 8.1 \\
\cline { 2 - 4 } & $\begin{array}{c}\text { 7-membered } \\
\text { endoperoxide } \\
\text { formation }\end{array}$ & $\mathrm{A}$ & $1.7 \times 10^{-2}$ \\
\hline
\end{tabular}

Table S 26. Calculated MC-TST reaction rate coefficients, $\mathrm{k}$, at $298.15 \mathrm{~K}$ for the unimolecular reactions of the hydroxy peroxy radicals formed from terpinolene $+\mathrm{OH}+\mathrm{O}_{2}$ (Figure $\mathrm{S} 15$ ). All values are calculated at the $\omega$ B97X-D/aug-cc-pVTZ level of theory with tunneling based on IRC end-points. The abstraction/addition site refer to the structures in Figure S 14, with "-OH" referring to abstraction of the hydrogen from the hydroxy group on the specified carbon atom.

\begin{tabular}{|c|c|c|c|}
\hline Peroxy Radical & Reaction type & $\begin{array}{c}\text { Abstraction/addition } \\
\text { site }\end{array}$ & $\mathrm{k}\left(\mathrm{s}^{-1}\right)$ \\
\hline \multirow{4}{*}{$\mathrm{T} 1$} & 1,5 H-shift & $\mathrm{E}$ & $2.9 \times 10^{-3}$ \\
\hline & 1,5-OH H-shift & $\mathrm{B}-\mathrm{OH}$ & $1.8 \times 10^{-3}$ \\
\hline & 1,5 H-shift & $\mathrm{C}$ & $2.8 \times 10^{-3}$ \\
\hline & $\begin{array}{l}\text { 6-membered } \\
\text { endoperoxide } \\
\text { formation }\end{array}$ & $\mathrm{D}$ & $7.1 \times 10^{-3}$ \\
\hline \multirow[b]{2}{*}{$\mathrm{T} 2$} & 1,5-OH H-shift & $\mathrm{B}-\mathrm{OH}$ & $5.1 \times 10^{-5}$ \\
\hline & $\begin{array}{l}\text { 6-membered } \\
\text { endoperoxide } \\
\text { formation }\end{array}$ & $\mathrm{D}$ & $1.3 \times 10^{-4}$ \\
\hline \multirow[b]{2}{*}{$\mathrm{T} 3$} & 1,5-OH H-shift & $\mathrm{A}-\mathrm{OH}$ & $4.6 \times 10^{-4}$ \\
\hline & $\begin{array}{l}\text { 5-membered } \\
\text { endoperoxide } \\
\text { formation }\end{array}$ & $\mathrm{D}$ & 0.30 \\
\hline \multirow{4}{*}{$\mathrm{T} 4$} & 1,5-OH H-shift & $\mathrm{A}-\mathrm{OH}$ & $4.2 \times 10^{-4}$ \\
\hline & 1,7 H-shift & $\mathrm{J}$ & 0.13 \\
\hline & $\begin{array}{c}\text { 5-membered } \\
\text { endoperoxide } \\
\text { formation }\end{array}$ & $\mathrm{D}$ & 1.2 \\
\hline & $\begin{array}{l}\text { 6-membered } \\
\text { endoperoxide } \\
\text { formation }\end{array}$ & $\mathrm{H}$ & $9.5 \times 10^{-3}$ \\
\hline \multirow{2}{*}{ T5 } & 1,4 H-shift & $\mathrm{C}$ & $1.2 \times 10^{-2}$ \\
\hline & 1,5 H-shift & $\mathrm{F}$ & $4.1 \times 10^{-2}$ \\
\hline
\end{tabular}




\begin{tabular}{|c|c|c|c|}
\hline & 1,5-OH H-shift & $\mathrm{H}-\mathrm{OH}$ & $1.3 \times 10^{-3}$ \\
\hline & $\begin{array}{l}\text { 5-membered } \\
\text { endoperoxide } \\
\text { formation }\end{array}$ & B & $1.5 \times 10^{-2}$ \\
\hline \multirow{5}{*}{ T6 } & 1,5-OH H-shift & $\mathrm{D}-\mathrm{OH}$ & $6.2 \times 10^{-4}$ \\
\hline & 1,5 H-shift & $\mathrm{C}$ & 4.9 \\
\hline & 1,6 H-shift & $\mathrm{F}$ & 0.66 \\
\hline & $\begin{array}{l}\text { 6-membered } \\
\text { endoperoxide } \\
\text { formation }\end{array}$ & B & 3.4 \\
\hline & $\begin{array}{l}\text { 7-membered } \\
\text { endoperoxide } \\
\text { formation }\end{array}$ & A & $1.6 \times 10^{-3}$ \\
\hline
\end{tabular}


Table S 27. Calculated reaction rate coefficients, $\mathrm{k}$, at $298.15 \mathrm{~K}$ for the unimolecular reactions of the hydroxy peroxy radicals formed from terpinolene $+\mathrm{OH}+\mathrm{O}_{2}$ (Figure $\mathrm{S} 15$ ). All values are calculated at the B3LYP/6$31+\mathrm{G}(\mathrm{d})$ level of theory with the symmetrical Eckart tunneling approach assuming thermoneutral reactions. The abstraction/addition site refer to the structures in Figure S 14, with "-OH" referring to abstraction of the hydrogen from the hydroxy group on the specified carbon atom. The reactions highlighted in bold are the ones also treated at a higher level of theory.

\begin{tabular}{|c|c|c|c|}
\hline Peroxy Radical & Reaction type & Abstraction/addition site & $\mathrm{k}\left(\mathrm{s}^{-1}\right)$ \\
\hline \multirow{10}{*}{$\mathrm{T} 1$} & 1,4 H-shift & B & $1.3 \times 10^{-10}$ \\
\hline & 1,4 H-shift & $\mathrm{G}$ & $9.7 \times 10^{-10}$ \\
\hline & 1,4 H-shift & $\mathrm{F}$ & $6.3 \times 10^{-9}$ \\
\hline & 1,5 H-shift & $\mathbf{E}$ & $2.8 \times 10^{-2}$ \\
\hline & 1,5-OH H-shift & B-OH & 2.5 \\
\hline & 1,5 H-shift & $\mathbf{C}$ & $3.4 \times 10^{-2}$ \\
\hline & $1,8 \mathrm{H}$-shift & I & $2.8 \times 10^{-6}$ \\
\hline & 1,8 H-shift & $\mathrm{J}$ & $1.3 \times 10^{-5}$ \\
\hline & $\begin{array}{l}\text { 6-membered } \\
\text { endoperoxide } \\
\text { formation }\end{array}$ & $\mathbf{D}$ & 0.56 \\
\hline & $\begin{array}{l}\text { 7-membered } \\
\text { endoperoxide } \\
\text { formation }\end{array}$ & $\mathrm{H}$ & $1.6 \times 10^{-4}$ \\
\hline \multirow{10}{*}{$\mathrm{T} 2$} & 1,4 H-shift & B & $2.3 \times 10^{-5}$ \\
\hline & 1,4 H-shift & $\mathrm{G}$ & $9.4 \times 10^{-10}$ \\
\hline & 1,4 H-shift & $\mathrm{F}$ & $1.5 \times 10^{-8}$ \\
\hline & 1,5 H-shift & $\mathrm{E}$ & $1.4 \times 10^{-3}$ \\
\hline & 1,5-OH H-shift & B-OH & 0.12 \\
\hline & 1,5 H-shift & $\mathrm{C}$ & $3.2 \times 10^{-3}$ \\
\hline & $1,8 \mathrm{H}$-shift & I & $6.9 \times 10^{-7}$ \\
\hline & 1,8 H-shift & $\mathrm{J}$ & $3.3 \times 10^{-7}$ \\
\hline & $\begin{array}{l}\text { 6-membered } \\
\text { endoperoxide } \\
\text { formation }\end{array}$ & $\mathbf{D}$ & $2.1 \times 10^{-2}$ \\
\hline & $\begin{array}{c}\text { 7-membered } \\
\text { endoperoxide } \\
\text { formation }\end{array}$ & $\mathrm{H}$ & $2.5 \times 10^{-6}$ \\
\hline \multirow{4}{*}{$\mathrm{T} 3$} & 1,4 H-shift & $\mathrm{C}$ & $3.0 \times 10^{-4}$ \\
\hline & $1,5 \mathrm{H}$-shift & $\mathrm{G}$ & $4.0 \times 10^{-6}$ \\
\hline & 1,5 H-shift & $\mathrm{F}$ & $3.3 \times 10^{-7}$ \\
\hline & 1,5-OH H-shift & A-OH & 0.22 \\
\hline
\end{tabular}




\begin{tabular}{|c|c|c|c|}
\hline & 1,6 H-shift & $\mathrm{E}$ & $9.8 \times 10^{-5}$ \\
\hline & 1,7 H-shift & I & $9.3 \times 10^{-14}$ \\
\hline & 1,7 H-shift & $\mathrm{J}$ & $3.7 \times 10^{-3}$ \\
\hline & $\begin{array}{l}\text { 5-membered } \\
\text { endoperoxide } \\
\text { formation }\end{array}$ & D & 10 \\
\hline & $\begin{array}{l}\text { 6-membered } \\
\text { endoperoxide } \\
\text { formation }\end{array}$ & $\mathrm{H}$ & $2.6 \times 10^{-3}$ \\
\hline \multirow{9}{*}{$\mathrm{T} 4$} & 1,4 H-shift & $\mathrm{C}$ & $3.7 \times 10^{-4}$ \\
\hline & 1,5 H-shift & $\mathrm{G}$ & $1.3 \times 10^{-6}$ \\
\hline & 1,5 H-shift & $\mathrm{F}$ & $1.7 \times 10^{-7}$ \\
\hline & 1,5-OH H-shift & A-OH & 0.60 \\
\hline & 1,6 H-shift & $\mathrm{E}$ & $2.4 \times 10^{-4}$ \\
\hline & 1,7 H-shift & I & $2.7 \times 10^{-14}$ \\
\hline & 1,7 H-shift & $\mathbf{J}$ & $5.0 \times 10^{-2}$ \\
\hline & $\begin{array}{l}\text { 5-membered } \\
\text { endoperoxide } \\
\text { formation }\end{array}$ & D & 89 \\
\hline & $\begin{array}{c}\text { 6-membered } \\
\text { endoperoxide } \\
\text { formation } \\
\end{array}$ & $\mathbf{H}$ & $2.7 \times 10^{-2}$ \\
\hline \multirow{10}{*}{$\mathrm{T} 5$} & 1,4 H-shift & $\mathrm{E}$ & $5.1 \times 10^{-8}$ \\
\hline & 1,4 H-shift & $\mathbf{C}$ & $4.2 \times 10^{-2}$ \\
\hline & 1,5 H-shift & I & $2.7 \times 10^{-6}$ \\
\hline & $1,5 \mathrm{H}$-shift & $\mathrm{J}$ & $4.5 \times 10^{-6}$ \\
\hline & 1,5 H-shift & $\mathbf{F}$ & 0.32 \\
\hline & 1,5-OH H-shift & H-OH & 0.92 \\
\hline & 1,5 H-shift & $\mathrm{B}$ & $2.1 \times 10^{-19}$ \\
\hline & 1,7 H-shift & $\mathrm{G}$ & $9.3 \times 10^{-12}$ \\
\hline & $\begin{array}{c}\text { 5-membered } \\
\text { endoperoxide } \\
\text { formation }\end{array}$ & B & 3.4 \\
\hline & $\begin{array}{c}\text { 6-membered } \\
\text { endoperoxide } \\
\text { formation }\end{array}$ & $\mathrm{A}-\mathrm{OH}$ & $3.9 \times 10^{-3}$ \\
\hline \multirow{2}{*}{ T6 } & 1,4 H-shift & I & $7.7 \times 10^{-10}$ \\
\hline & 1,4 H-shift & $\mathrm{J}$ & $7.5 \times 10^{-10}$ \\
\hline
\end{tabular}




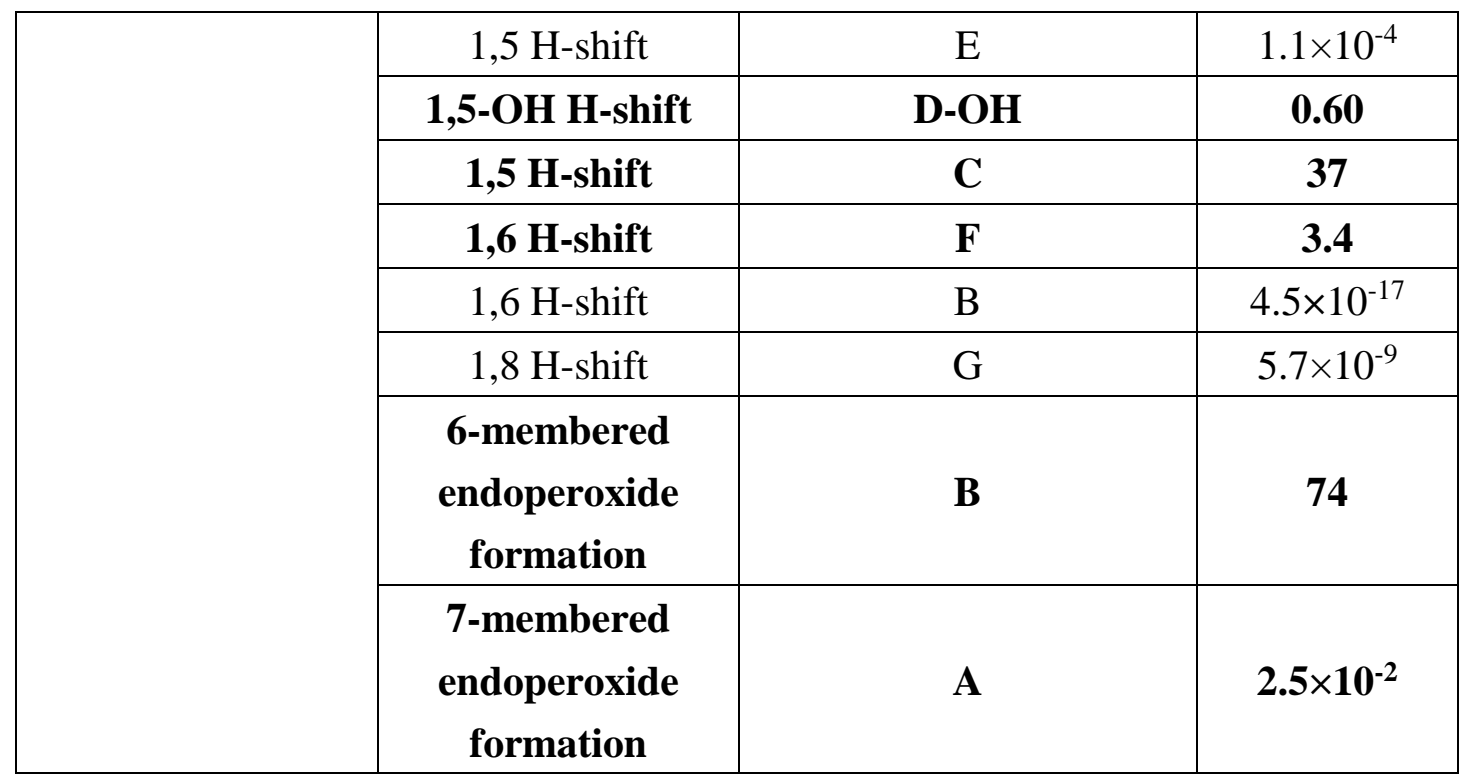

Table S 28. CCSD(T)-F12a/VDZ-F12 (abbreviated F12) and $\omega$ B97X-D/aug-cc-pVTZ barrier heights ( $\mathrm{E}_{0}$, in $\mathrm{kcal} / \mathrm{mol})$, summed partition function ratios $\left(\mathrm{Q}_{\mathrm{TS}} / \mathrm{Q}_{\mathrm{R}}\right)$ and Eckart tunneling coefficients $(\kappa)$ used for calculating the corresponding MC-TST rate coefficients in the preceding tables for terpinolene.

\begin{tabular}{|c|c|c|c|c|c|c|c|}
\hline $\begin{array}{l}\text { Peroxy } \\
\text { Radical }\end{array}$ & Reaction type & $\begin{array}{l}\text { Abstraction/ } \\
\text { addition site }\end{array}$ & $\begin{array}{c}\mathrm{E}_{0} \\
(\mathrm{~F} 12) \\
\end{array}$ & $\begin{array}{c}\kappa \\
(\mathrm{F} 12)\end{array}$ & $\begin{array}{c}\mathrm{E}_{0} \\
(\omega \mathrm{B} 97 \mathrm{X}-\mathrm{D})\end{array}$ & $\mathrm{Q}_{\mathrm{TS}} / \mathrm{Q}_{\mathrm{R}}$ & $\begin{array}{c}\kappa \\
(\omega \mathrm{B} 97 \mathrm{X}-\mathrm{D})\end{array}$ \\
\hline \multirow{4}{*}{$\mathrm{T} 1$} & 1,5 H-shift & $\mathrm{E}$ & 23.0 & 868.2 & 23.8 & $1.54 \times 10^{-1}$ & 847.4 \\
\hline & $\begin{array}{c}\text { 1,5-OH H- } \\
\text { shift }\end{array}$ & $\mathrm{B}-\mathrm{OH}$ & - & - & 20.1 & $1.97 \times 10^{-1}$ & 0.8 \\
\hline & $1,5 \mathrm{H}$-shift & $\mathrm{C}$ & 22.7 & 528.5 & 23.4 & $1.30 \times 10^{-1}$ & 517.2 \\
\hline & $\begin{array}{c}\text { 6-membered } \\
\text { endoperoxide } \\
\text { formation }\end{array}$ & $\mathrm{D}$ & 18.0 & 1.4 & 19.2 & $9.36 \times 10^{-2}$ & 1.4 \\
\hline \multirow[b]{2}{*}{$\mathrm{T} 2$} & $\begin{array}{c}\text { 1,5-OH H- } \\
\text { shift }\end{array}$ & $\mathrm{B}-\mathrm{OH}$ & - & - & 22.4 & $9.75 \times 10^{-2}$ & 2.0 \\
\hline & $\begin{array}{c}\text { 6-membered } \\
\text { endoperoxide } \\
\text { formation }\end{array}$ & $\mathrm{D}$ & 20.7 & 1.4 & 21.9 & $1.55 \times 10^{-1}$ & 1.4 \\
\hline \multirow[b]{2}{*}{$\mathrm{T} 3$} & $\begin{array}{c}\text { 1,5-OH H- } \\
\text { shift }\end{array}$ & $\mathrm{A}-\mathrm{OH}$ & - & - & 21.1 & $2.03 \times 10^{-1}$ & 1.1 \\
\hline & $\begin{array}{c}\text { 5-membered } \\
\text { endoperoxide } \\
\text { formation }\end{array}$ & $\mathrm{D}$ & 15.9 & 1.3 & 16.7 & $7.19 \times 10^{-2}$ & 1.3 \\
\hline \multirow[t]{2}{*}{$\mathrm{T} 4$} & $\begin{array}{c}\text { 1,5-OH H- } \\
\text { shift }\end{array}$ & $\mathrm{A}-\mathrm{OH}$ & - & - & 21.1 & $1.25 \times 10^{-1}$ & 1.5 \\
\hline & 1,7 H-shift & $\mathrm{J}$ & 19.4 & 713.7 & 20.5 & $3.60 \times 10^{-2}$ & 636.3 \\
\hline
\end{tabular}




\begin{tabular}{|c|c|c|c|c|c|c|c|}
\hline & $\begin{array}{c}\text { 5-membered } \\
\text { endoperoxide } \\
\text { formation }\end{array}$ & D & 15.4 & 1.3 & 16.4 & $1.48 \times 10^{-1}$ & 1.3 \\
\hline & $\begin{array}{c}\text { 6-membered } \\
\text { endoperoxide } \\
\text { formation }\end{array}$ & $\mathrm{H}$ & 16.1 & 1.3 & 18.7 & $6.33 \times 10^{-2}$ & 1.3 \\
\hline \multirow{4}{*}{ T5 } & 1,4 H-shift & $\mathrm{C}$ & 26.3 & 46538.4 & 25.5 & $3.56 \times 10^{-1}$ & 26984.9 \\
\hline & $1,5 \mathrm{H}$-shift & $\mathrm{F}$ & 21.7 & 601.6 & 22.2 & $2.66 \times 10^{-1}$ & 496.6 \\
\hline & $\begin{array}{c}\text { 1,5-OH H- } \\
\text { shift }\end{array}$ & $\mathrm{H}-\mathrm{OH}$ & - & - & 20.3 & $1.58 \times 10^{-1}$ & 1.0 \\
\hline & $\begin{array}{c}\text { 5-membered } \\
\text { endoperoxide } \\
\text { formation }\end{array}$ & B & 18.5 & 1.5 & 19.4 & $2.71 \times 10^{-1}$ & 1.5 \\
\hline \multirow{5}{*}{ T6 } & $\begin{array}{c}\text { 1,5-OH H- } \\
\text { shift }\end{array}$ & $\mathrm{D}-\mathrm{OH}$ & - & - & 20.6 & $1.29 \times 10^{-1}$ & 1.0 \\
\hline & 1,5 H-shift & $\mathrm{C}$ & 20.1 & 1138.9 & 19.8 & $3.28 \times 10^{-1}$ & 835.5 \\
\hline & 1,6 H-shift & $\mathrm{F}$ & 19.5 & 121.2 & 19.3 & $1.39 \times 10^{-1}$ & 105.1 \\
\hline & $\begin{array}{c}\text { 6-membered } \\
\text { endoperoxide } \\
\text { formation }\end{array}$ & B & 15.2 & 1.4 & 15.7 & $1.32 \times 10^{-1}$ & 1.4 \\
\hline & $\begin{array}{c}\text { 7-membered } \\
\text { endoperoxide } \\
\text { formation }\end{array}$ & A & 18.5 & 1.3 & 19.9 & $7.01 \times 10^{-2}$ & 1.3 \\
\hline
\end{tabular}


Table S 29. B3LYP/6-31+G(d) barrier heights $\left(\mathrm{E}_{0}\right.$, in kcal/mol), summed partition function ratios $\left(\mathrm{Q}_{\mathrm{TS}} / \mathrm{Q}_{\mathrm{R}}\right)$ and Eckart tunneling coefficients $(\kappa)$ used for calculating the corresponding MC-TST rate coefficients in the preceding tables for terpinolene.

\begin{tabular}{|c|c|c|c|c|c|}
\hline $\begin{array}{l}\text { Peroxy } \\
\text { radical }\end{array}$ & Reaction type & $\begin{array}{l}\text { Abstraction/ } \\
\text { addition site }\end{array}$ & $\begin{array}{c}\mathrm{E}_{0} \\
(\mathrm{~B} 3 \mathrm{LYP})\end{array}$ & $\mathrm{Q}_{\mathrm{TS}} / \mathrm{Q}_{\mathrm{R}}$ & $\begin{array}{c}\kappa \\
(\mathrm{B} 3 \mathrm{LYP})\end{array}$ \\
\hline \multirow{10}{*}{$\mathrm{T} 1$} & 1,4 H-shift & $\mathrm{B}$ & 35.1 & $1.98 \times 10^{-1}$ & 6067.8 \\
\hline & 1,4 H-shift & $\mathrm{G}$ & 36.3 & $4.12 \times 10^{-1}$ & 163816.4 \\
\hline & 1,4 H-shift & $\mathrm{F}$ & 34.6 & $3.64 \times 10^{-1}$ & 66525.0 \\
\hline & 1,5 H-shift & $\mathrm{E}$ & 22.0 & $2.59 \times 10^{-1}$ & 213.9 \\
\hline & 1,5-OH H-shift & $\mathrm{B}-\mathrm{OH}$ & 17.1 & $1.80 \times 10^{-1}$ & 7.6 \\
\hline & 1,5 H-shift & $\mathrm{C}$ & 21.6 & $2.09 \times 10^{-1}$ & 178.8 \\
\hline & 1,8 H-shift & I & 27.0 & $7.94 \times 10^{-2}$ & 340.1 \\
\hline & 1,8 H-shift & $\mathrm{J}$ & 25.6 & $5.86 \times 10^{-2}$ & 203.7 \\
\hline & $\begin{array}{l}\text { 6-membered } \\
\text { endoperoxide } \\
\text { formation }\end{array}$ & $\mathrm{D}$ & 16.3 & $6.90 \times 10^{-2}$ & 1.2 \\
\hline & $\begin{array}{c}\text { 7-membered } \\
\text { endoperoxide } \\
\text { formation }\end{array}$ & $\mathrm{H}$ & 20.3 & $1.50 \times 10^{-2}$ & 1.2 \\
\hline \multirow{10}{*}{$\mathrm{T} 2$} & 1,4 H-shift & $\mathrm{B}$ & 27.1 & $3.12 \times 10^{-1}$ & 882.5 \\
\hline & 1,4 H-shift & $\mathrm{G}$ & 36.8 & $3.81 \times 10^{-1}$ & 368192.0 \\
\hline & 1,4 H-shift & $\mathrm{F}$ & 34.3 & $4.75 \times 10^{-1}$ & 70903.0 \\
\hline & 1,5 H-shift & $\mathrm{E}$ & 24.3 & $4.40 \times 10^{-1}$ & 361.1 \\
\hline & 1,5-OH H-shift & $\mathrm{B}-\mathrm{OH}$ & 19.1 & $1.68 \times 10^{-1}$ & 12.0 \\
\hline & 1,5 H-shift & $\mathrm{C}$ & 23.9 & $3.03 \times 10^{-1}$ & 542.6 \\
\hline & 1,8 H-shift & I & 28.0 & $7.86 \times 10^{-2}$ & 453.0 \\
\hline & 1,8 H-shift & $\mathrm{J}$ & 28.1 & $4.90 \times 10^{-2}$ & 404.9 \\
\hline & $\begin{array}{l}\text { 6-membered } \\
\text { endoperoxide } \\
\text { formation }\end{array}$ & $\mathrm{D}$ & 19.2 & $2.91 \times 10^{-1}$ & 1.3 \\
\hline & $\begin{array}{l}\text { 7-membered } \\
\text { endoperoxide } \\
\text { formation }\end{array}$ & $\mathrm{H}$ & 23.6 & $6.59 \times 10^{-2}$ & 1.2 \\
\hline \multirow{5}{*}{ T3 } & 1,4 H-shift & $\mathrm{C}$ & 24.6 & $9.05 \times 10^{-2}$ & 552.9 \\
\hline & 1,5 H-shift & $\mathrm{G}$ & 26.1 & $7.86 \times 10^{-2}$ & 116.3 \\
\hline & 1,5 H-shift & $\mathrm{F}$ & 28.6 & $2.00 \times 10^{-1}$ & 242.1 \\
\hline & 1,5-OH H-shift & $\mathrm{A}-\mathrm{OH}$ & 18.2 & $1.12 \times 10^{-1}$ & 6.5 \\
\hline & 1,6 H-shift & $\mathrm{E}$ & 24.6 & $5.70 \times 10^{-2}$ & 285.1 \\
\hline
\end{tabular}




\begin{tabular}{|c|c|c|c|c|c|}
\hline & 1,7 H-shift & I & 35.8 & $4.07 \times 10^{-3}$ & 694.3 \\
\hline & 1,7 H-shift & $\mathrm{J}$ & 21.3 & $9.04 \times 10^{-3}$ & 279.4 \\
\hline & $\begin{array}{l}\text { 5-membered } \\
\text { endoperoxide } \\
\text { formation }\end{array}$ & $\mathrm{D}$ & 14.7 & $7.50 \times 10^{-2}$ & 1.2 \\
\hline & $\begin{array}{l}\text { 6-membered } \\
\text { endoperoxide } \\
\text { formation }\end{array}$ & $\mathrm{H}$ & 18.5 & $1.26 \times 10^{-2}$ & 1.2 \\
\hline \multirow{9}{*}{$\mathrm{T} 4$} & 1,4 H-shift & $\mathrm{C}$ & 26.4 & $6.92 \times 10^{-1}$ & 2083.9 \\
\hline & 1,5 H-shift & $\mathrm{G}$ & 27.1 & $1.61 \times 10^{-1}$ & 94.4 \\
\hline & 1,5 H-shift & $\mathrm{F}$ & 30.0 & $3.30 \times 10^{-1}$ & 825.2 \\
\hline & 1,5-OH H-shift & $\mathrm{A}-\mathrm{OH}$ & 18.0 & $1.65 \times 10^{-1}$ & 9.4 \\
\hline & 1,6 H-shift & $E$ & 26.1 & $3.75 \times 10^{-1}$ & 1302.1 \\
\hline & 1,7 H-shift & $\mathrm{I}$ & 38.3 & $8.10 \times 10^{-3}$ & 6381.4 \\
\hline & 1,7 H-shift & $\mathrm{J}$ & 20.2 & $2.38 \times 10^{-2}$ & 235.3 \\
\hline & $\begin{array}{l}\text { 5-membered } \\
\text { endoperoxide } \\
\text { formation }\end{array}$ & $\mathrm{D}$ & 13.7 & $1.40 \times 10^{-1}$ & 1.2 \\
\hline & $\begin{array}{l}\text { 6-membered } \\
\text { endoperoxide } \\
\text { formation }\end{array}$ & $\mathrm{H}$ & 17.7 & $3.49 \times 10^{-2}$ & 1.2 \\
\hline \multirow{10}{*}{ T5 } & 1,4 H-shift & $\mathrm{E}$ & 33.3 & $6.33 \times 10^{-1}$ & 30855.4 \\
\hline & 1,4 H-shift & $\mathrm{C}$ & 22.6 & $2.53 \times 10^{-1}$ & 1027.6 \\
\hline & $1,5 \mathrm{H}$-shift & I & 26.4 & $1.51 \times 10^{-1}$ & 62.8 \\
\hline & 1,5 H-shift & $\mathrm{J}$ & 25.9 & $1.36 \times 10^{-1}$ & 47.5 \\
\hline & 1,5 H-shift & $\mathrm{F}$ & 21.0 & $3.68 \times 10^{-1}$ & 324.3 \\
\hline & 1,5-OH H-shift & $\mathrm{H}-\mathrm{OH}$ & 17.3 & $1.69 \times 10^{-1}$ & 4.5 \\
\hline & 1,5 H-shift & $\mathrm{B}$ & 50.6 & $2.19 \times 10^{-1}$ & 1915462.1 \\
\hline & 1,7 H-shift & $\mathrm{G}$ & 35.7 & $4.83 \times 10^{-2}$ & 4873.4 \\
\hline & $\begin{array}{l}\text { 5-membered } \\
\text { endoperoxide } \\
\text { formation }\end{array}$ & B & 16.0 & $2.17 \times 10^{-1}$ & 1.3 \\
\hline & $\begin{array}{l}\text { 6-membered } \\
\text { endoperoxide } \\
\text { formation }\end{array}$ & $\mathrm{A}-\mathrm{OH}$ & 19.7 & $1.40 \times 10^{-1}$ & 1.3 \\
\hline \multirow{3}{*}{ T6 } & 1,4 H-shift & $\mathrm{I}$ & 36.2 & $2.72 \times 10^{-1}$ & 144060.8 \\
\hline & 1,4 H-shift & $\mathrm{J}$ & 36.1 & $2.27 \times 10^{-1}$ & 154275.3 \\
\hline & 1,5 H-shift & $\mathrm{E}$ & 25.1 & $2.92 \times 10^{-1}$ & 156.5 \\
\hline
\end{tabular}




\begin{tabular}{|c|c|c|c|c|c|}
\hline & $1,5-\mathrm{OH}$ H-shift & $\mathrm{D}-\mathrm{OH}$ & 17.4 & $1.24 \times 10^{-1}$ & 4.1 \\
\cline { 2 - 6 } & $1,5 \mathrm{H}$-shift & $\mathrm{C}$ & 17.6 & $2.16 \times 10^{-1}$ & 200.8 \\
\cline { 2 - 6 } & $1,6 \mathrm{H}$-shift & $\mathrm{F}$ & 18.3 & $1.32 \times 10^{-1}$ & 99.4 \\
\hline $1,6 \mathrm{H}$-shift & $\mathrm{B}$ & 34.4 & $1.21 \times 10^{-1}$ & 20142.5 \\
\hline & $\mathrm{G}$ & 13.8 & $1.14 \times 10^{-1}$ & 1.3 \\
\cline { 2 - 6 } & $\begin{array}{c}\text { 6-membered } \\
\text { endoperoxide } \\
\text { formation }\end{array}$ & $\mathrm{B}$ & 18.1 & $5.68 \times 10^{-2}$ & 1.2 \\
\hline $\begin{array}{c}\text { 7-membered } \\
\text { endoperoxide } \\
\text { formation }\end{array}$ & $\mathrm{A}$ & $1.00 \times 10^{-1}$ & 1212.0 \\
\hline
\end{tabular}




\section{S4 Modeling of the Ring-opening in Camphene}

For $\alpha$-pinene and $\beta$-pinene, calculations and experiments suggest that some fraction undergoes ringopening from the vibrationally-excited hydroxy alkyl radical formed by addition of the hydroxyl radicals to one of the carbon atoms in the double bond. ${ }^{2}$ Camphene similarly has a secondary ring and could undergo various ring-openings (Figure S 16-Figure S 17).

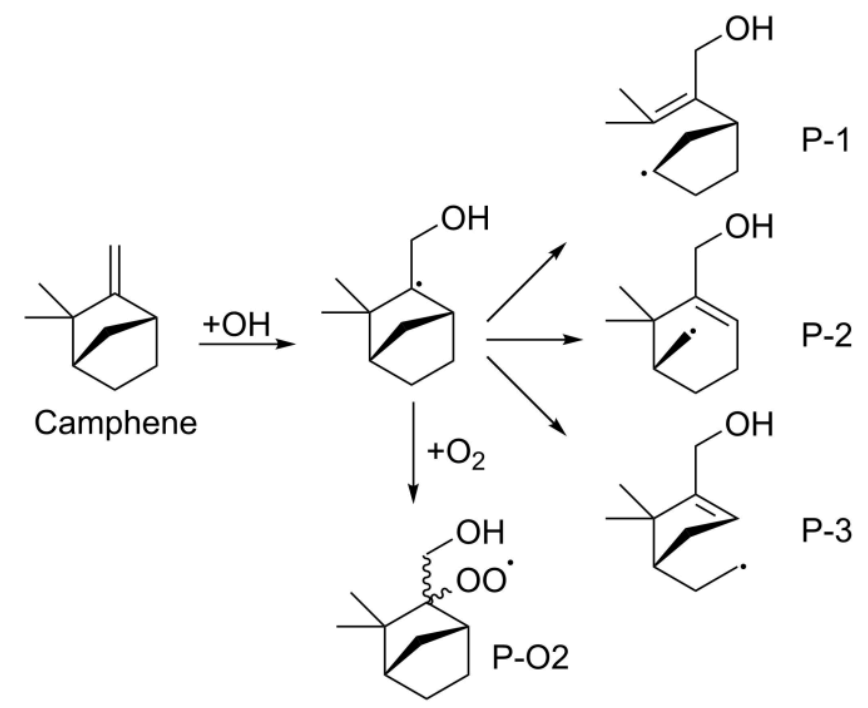

Figure S 16. Ring-opening mechanisms of camphene following $\mathrm{OH}$ addition to the terminal unsaturated carbon atom.

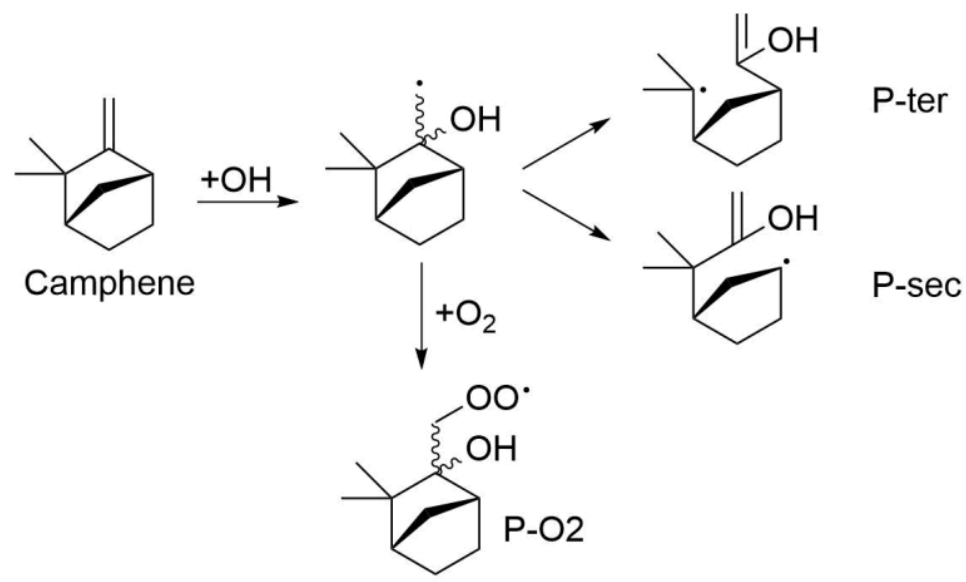

Figure S 17. Ring-opening mechanisms of camphene following $\mathrm{OH}$ addition to the internal unsaturated carbon atom. $\mathrm{OH}$ may add to either of the two sides of the ring and both options are considered.

Conformational sampling and subsequent computational steps are done as described for the calculation of reaction rate coefficients and in the approach by Møller et al. ${ }^{1}$ The stationary points are optimized using $\omega B 97 X-D / a u g-c c-p V T Z$. For the stationary points excluding the products, electronic energies were calculated using CCSD(T)-F12a/cc-pVDZ-F12. The ZPVE is calculated using $\omega B 97 X-D / a u g-c c-p V T Z$. For the products, which do not impact the simulations, the relative $\omega$ B97X-D/aug-cc-pVTZ energies are used. The energy diagrams for the pathways shown in Figure S 16 and Figure S 17 are shown in Figure S 18Figure S 20. 


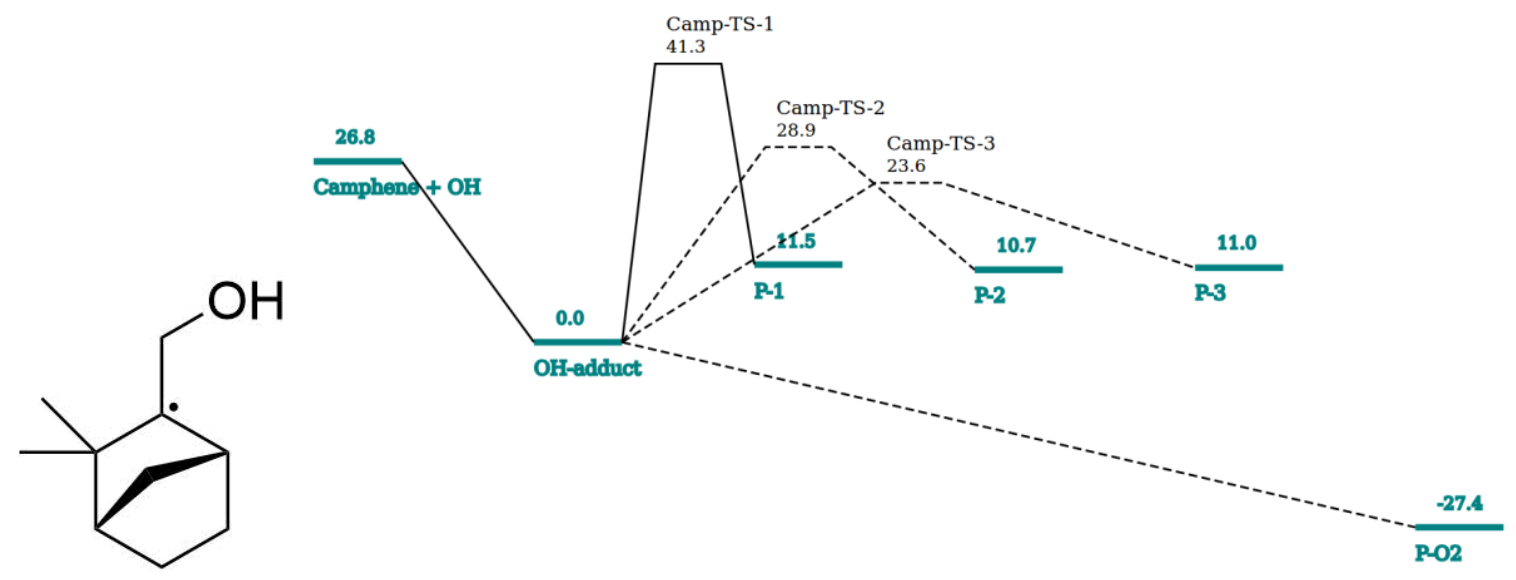

Figure S 18. Energy diagram of the ring-opening pathways following $\mathrm{OH}$-addition to the terminal

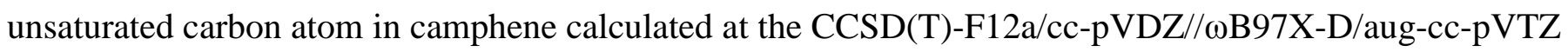
(non-products) and $\omega \mathrm{B} 97 \mathrm{X}-\mathrm{D} / \mathrm{aug}-\mathrm{cc}-\mathrm{pVTZ}$ (products) levels. All energies are zero-point corrected electronic energies in $\mathrm{kcal} / \mathrm{mol}$.
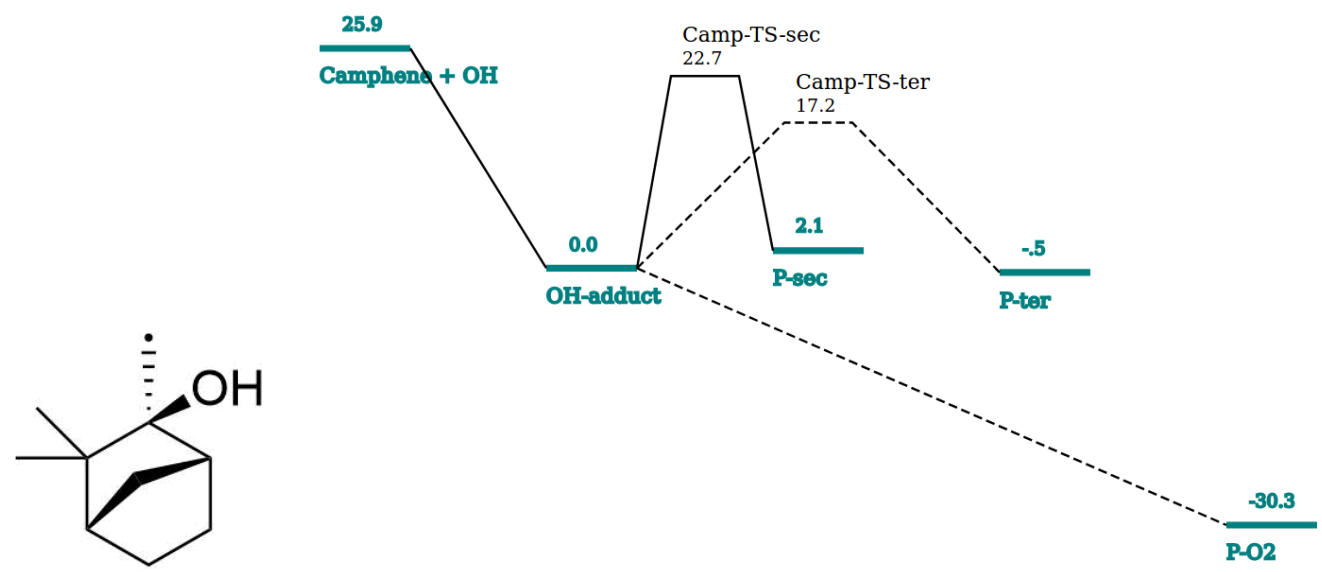

Figure S 19. Energy diagram of the ring-opening pathways following $\mathrm{OH}$-addition to the internal unsaturated carbon atom in camphene and $\mathrm{OH}$ pointing out of the plane calculated at the CCSD(T)-F12a/ccpVDZ// $\omega$ B97X-D/aug-cc-pVTZ (non-products) and $\omega$ B97X-D/aug-cc-pVTZ (products) levels. All energies are zero-point corrected electronic energies in $\mathrm{kcal} / \mathrm{mol}$. 


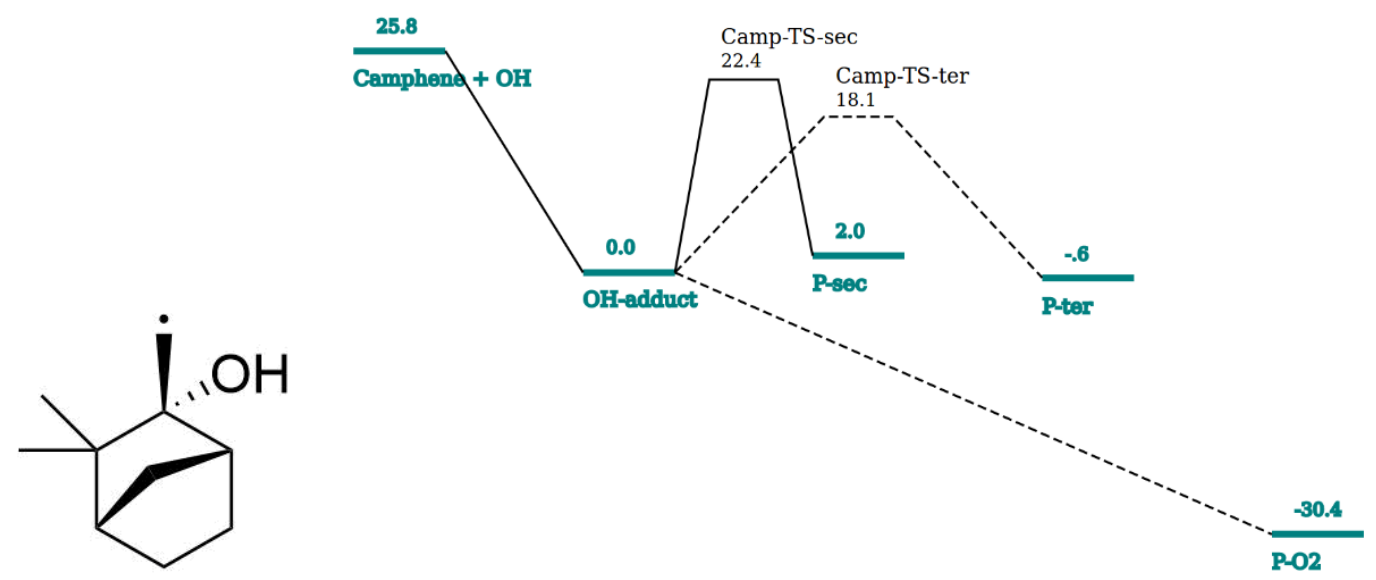

Figure S 20. Energy diagram of the ring-opening pathways following $\mathrm{OH}$-addition to the internal unsaturated carbon atom in camphene and $\mathrm{OH}$ pointing into the plane calculated at the CCSD(T)-F12a/cc-

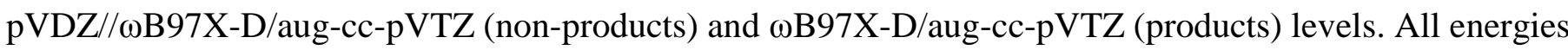
are zero-point corrected electronic energies in $\mathrm{kcal} / \mathrm{mol}$.

The RRKM simulations are run using the following parameters, which are very similar to those used in the corresponding modeling of $\alpha$-pinene and $\beta$-pinene: ${ }^{2}$

- $\mathrm{k}($ camphene+OH, $298 \mathrm{~K})=5.1 \times 10^{-11} \mathrm{~cm}^{3}$ molecule $\mathrm{s}^{-1} \mathrm{~s}^{-1,4}$

- $[\mathrm{OH}]=1 \times 10^{12}$ molecules $\mathrm{cm}^{-3}$. Set extremely high to better visualize the process. However, the product yields of the simulation are independent of this value and the same result is obtained using $[\mathrm{OH}]=1 \times 10^{6}$ molecules $\mathrm{cm}^{-3}$ corresponding to the estimated global average.

- $\mathrm{k}\left(\mathrm{R} \cdot+\mathrm{O}_{2}\right)=14 \times 10^{-12} \mathrm{~cm}^{3}$ molecule $\mathrm{s}^{-1}$. Value for cyclohexanyl $+\mathrm{O}_{2} .^{5}$ The exact value for this rate coefficient is not important, as long as the pseudo-first order rate coefficient for the $\mathrm{O}_{2}$-addition is significantly faster than the thermalized ring-opening and slower than the vibrational relaxation $\left(\sim 10^{10}\right.$ $\left.\mathrm{s}^{-1}\right)$.

- Exponential energy decay with an average energy transfer per collision $\left(\Delta E_{\text {down }}=225 \mathrm{~cm}^{-1}\right)$ based on values for similar simulations using $\mathrm{N}_{2}$ as the bath gas. ${ }^{6-7}$

- Lennard-Jones parameters for the camphene species: $\sigma=6.5 \AA, \varepsilon / k_{B}=600 .^{7}$

- Bath gas $=\mathrm{N}_{2}(\sigma=3.919 \AA, \varepsilon / \mathrm{kb}=91.85){ }^{8}$

- $\mathrm{P}=760$ Torr, $\mathrm{T}=298.15 \mathrm{~K}$

- Grain size $=100 \mathrm{~cm}^{-1}$ and energy grain span above highest stationary point $=50 \mathrm{k}_{\mathrm{B}} \mathrm{T}$.

- The products of the ring-opening reactions are modelled as "sinks" to avoid back reaction.

As can be seen in Figure S 21-Figure S 23, the sole product in the RRKM modeling of the camphene $+\mathrm{OH}$ oxidation pathways is the hydroxy peroxy radicals formed by $\mathrm{O}_{2}$-addition (P-O2), i.e. ring-opening does not seem to occur with any appreciable yield. For the terminal $\mathrm{OH}$-addition, the very high barriers mean that even if the model is rerun with a barrier for the lowest TS that is $5 \mathrm{kcal} / \mathrm{mol}$ lower, no ring-opening seems to occur. For the internal $\mathrm{OH}$-addition, the result is more sensitive to the barrier height. Still, decreasing the barrier height by $2 \mathrm{kcal} / \mathrm{mol}$ increases the yield of the ring-opening product to less than $3 \%$. 
The ring-opening is competitive in $\alpha$-pinene and $\beta$-pinene as the barrier for opening of the smaller 4-membered ring is only about $14 \mathrm{kcal} / \mathrm{mol}$ compared to $17 \mathrm{kcal} / \mathrm{mol}$ for the lowest-energy barrier in camphene. A greater amount of ring-strain in $\alpha$ - and $\beta$-pinene compared to camphene is in line with a lower energy for the latter (Table S 30).

\section{Species Populations}

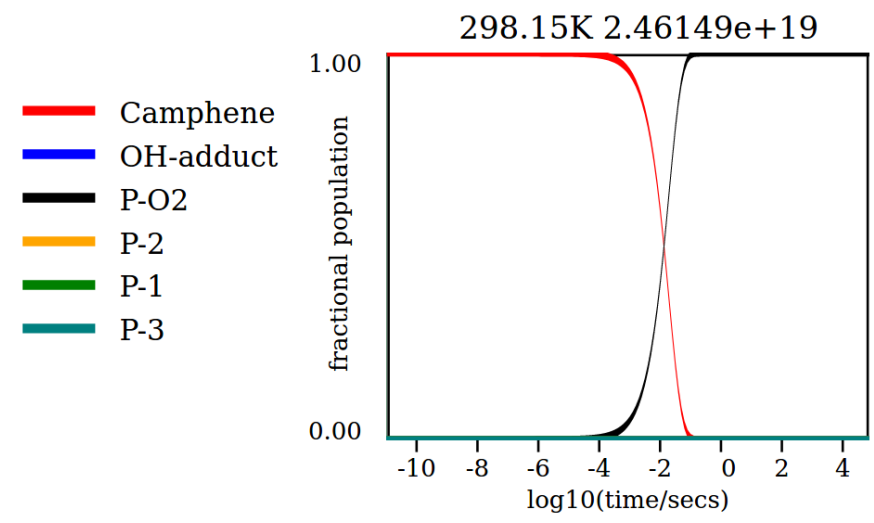

Figure $\mathrm{S} 21$. Product distribution for the camphene $+\mathrm{OH}$ oxidation pathways by $\mathrm{OH}$-addition to the terminal unsaturated carbon atom shown in Figure S 16 and Figure S 18.

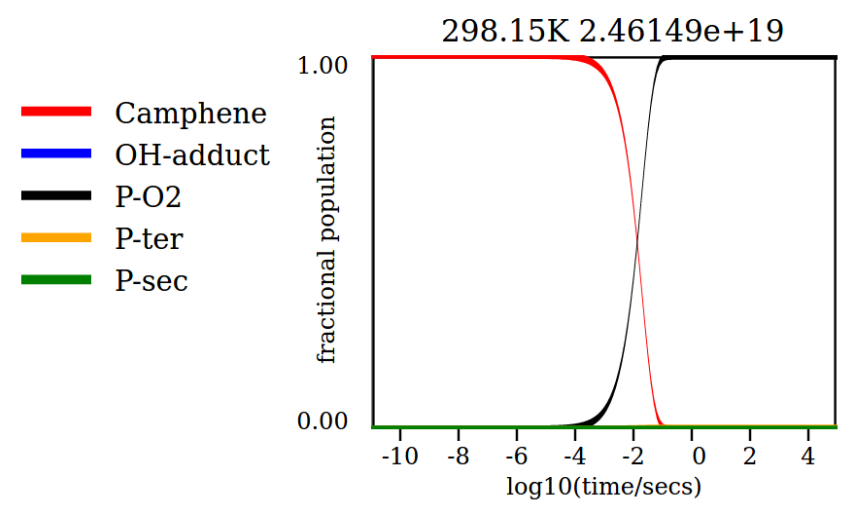

Figure S 22. Product distribution for the camphene $+\mathrm{OH}$ oxidation pathways by $\mathrm{OH}$-addition to the internal unsaturated carbon atom in camphene and $\mathrm{OH}$ pointing out of the plane shown in Figure $\mathrm{S} 17$ and Figure $\mathrm{S}$ 19.

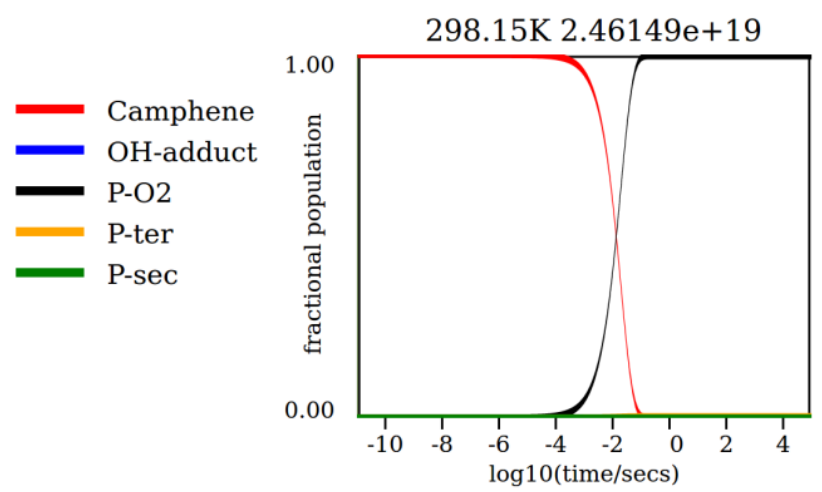

Figure S 23. Product distribution for the camphene $+\mathrm{OH}$ oxidation pathways by $\mathrm{OH}$-addition to the internal unsaturated carbon atom in camphene and $\mathrm{OH}$ pointing into the plane shown in Figure S 17 and Figure S 20. 
Table S 30. Relative energies (CCSD(T)-F12a electronic energy + $\omega$ B97X-D/aug-cc-pVTZ ZPVE, in $\mathrm{kcal} / \mathrm{mol}$ ) of three monoterpenes.

\begin{tabular}{|c|c|}
\hline Monoterpene & Relative energy \\
\hline$\alpha$-pinene & 11.83 \\
\hline$\beta$-pinene & 14.39 \\
\hline Camphene & 0.00 \\
\hline
\end{tabular}




\section{S5 Temperature-Dependence of Reaction Rate Coefficients}

The temperature-dependent rate coefficients, $\mathrm{k}$, are given by the expression: ${ }^{9-10}$

$$
k=A \mathrm{e}^{-B / T} \mathrm{e}^{C / T^{3}}
$$

Table S 31. Temperature-dependency parameters of all the reactions rate coefficients that have been calculated at the CCSD(T)-F12a/VDZ-F12 level.

\begin{tabular}{|c|c|c|c|c|c|c|}
\hline Compound & $\begin{array}{l}\text { Peroxy } \\
\text { radical }\end{array}$ & Reaction type & Addition site & A & B & $\mathrm{C}$ \\
\hline \multirow{9}{*}{$\alpha$-pinene } & \multirow{5}{*}{$\mathrm{A} 1$} & $1,5 \mathrm{H}$-shift & $E$ & $6.121 \mathrm{E}+11$ & $9.746 \mathrm{E}+03$ & $1.499 \mathrm{E}+08$ \\
\hline & & 1,5 H-shift & $\mathrm{C}$ & $1.576 \mathrm{E}+12$ & $9.859 \mathrm{E}+03$ & $1.39 \mathrm{E}+08$ \\
\hline & & $1,6 \mathrm{H}$-shift & $\mathrm{B}$ & $1.710 \mathrm{E}+13$ & $9.034 \mathrm{E}+03$ & $3.308 \mathrm{E}+07$ \\
\hline & & $\begin{array}{c}\text { 6-membered } \\
\text { endoperoxide } \\
\text { formation }\end{array}$ & $\mathrm{F}$ & $1.075 \mathrm{E}+12$ & 7.999E+03 & $7.113 \mathrm{E}+06$ \\
\hline & & $\begin{array}{c}\text { 7-membered } \\
\text { endoperoxide } \\
\text { formation } \\
\end{array}$ & A & $5.856 \mathrm{E}+11$ & $9.503 \mathrm{E}+03$ & $6.425 \mathrm{E}+06$ \\
\hline & \multirow{4}{*}{ A10 } & $1,5 \mathrm{H}$-shift & $\mathrm{E}$ & $5.813 \mathrm{E}+10$ & $9.310 \mathrm{E}+03$ & $1.213 \mathrm{E}+08$ \\
\hline & & 1,5 H-shift & $\mathrm{C}$ & $1.576 \mathrm{E}+12$ & $1.261 \mathrm{E}+04$ & $6.782 \mathrm{E}+07$ \\
\hline & & $\begin{array}{c}\text { 6-membered } \\
\text { endoperoxide } \\
\text { formation }\end{array}$ & $\mathrm{F}$ & $1.092 \mathrm{E}+10$ & $1.722 \mathrm{E}+04$ & $6.142 \mathrm{E}+06$ \\
\hline & & $\begin{array}{c}\text { 7-membered } \\
\text { endoperoxide } \\
\text { formation }\end{array}$ & A & $1.202 \mathrm{E}+10$ & $6.748 \mathrm{E}+03$ & $4.381 \mathrm{E}+06$ \\
\hline \multirow{6}{*}{$\beta$-pinene } & B2 & 1,5 H-shift & $\mathrm{B}$ & $2.971 \mathrm{E}+12$ & $1.029 \mathrm{E}+04$ & $8.656 \mathrm{E}+07$ \\
\hline & \multirow{5}{*}{ B5 } & 1,5 H-shift & $\mathrm{E}$ & $7.015 \mathrm{E}+11$ & $9.670 \mathrm{E}+03$ & $1.460 \mathrm{E}+08$ \\
\hline & & 1,5 H-shift & $\mathrm{C}$ & $2.732 \mathrm{E}+12$ & $1.292 \mathrm{E}+04$ & $7.525 \mathrm{E}+07$ \\
\hline & & 1,6 H-shift & B & $2.716 \mathrm{E}+11$ & $9.561 \mathrm{E}+03$ & $1.178 \mathrm{E}+08$ \\
\hline & & $\begin{array}{c}\text { 6-membered } \\
\text { endoperoxide } \\
\text { formation }\end{array}$ & $\mathrm{F}$ & $1.101 \mathrm{E}+10$ & $6.559 \mathrm{E}+03$ & $6.678 \mathrm{E}+06$ \\
\hline & & $\begin{array}{c}\text { 7-membered } \\
\text { endoperoxide } \\
\text { formation }\end{array}$ & A & $8.059 \mathrm{E}+09$ & $7.766 \mathrm{E}+03$ & $5.084 \mathrm{E}+06$ \\
\hline \multirow{6}{*}{ Limonene } & \multirow{6}{*}{ L1 } & \multirow{2}{*}{ 1,5 H-shift } & $\mathrm{C}(\mathrm{R}, \mathrm{R})$ & $5.055 \mathrm{E}+11$ & $9.104 \mathrm{E}+03$ & $1.313 \mathrm{E}+08$ \\
\hline & & & $\mathrm{C}(\mathrm{R}, \mathrm{S})$ & $4.087 \mathrm{E}+12$ & $1.028 \mathrm{E}+04$ & $1.273 \mathrm{E}+08$ \\
\hline & & \multirow{2}{*}{ 1,6 H-shift } & $\mathrm{F}(\mathrm{R}, \mathrm{R})$ & $8.100 \mathrm{E}+11$ & $9.974 \mathrm{E}+03$ & $1.163 \mathrm{E}+08$ \\
\hline & & & $\mathrm{F}(\mathrm{R}, \mathrm{S})$ & $5.428 \mathrm{E}+11$ & $9.392 \mathrm{E}+03$ & $1.086 \mathrm{E}+08$ \\
\hline & & \multirow{2}{*}{$\begin{array}{l}\text { 6-membered } \\
\text { endoperoxide }\end{array}$} & $\mathrm{B}(\mathrm{R}, \mathrm{R})$ & $1.040 \mathrm{E}+11$ & $7.221 \mathrm{E}+03$ & $5.739 \mathrm{E}+06$ \\
\hline & & & $\mathrm{B}(\mathrm{R}, \mathrm{S})$ & $7.173 \mathrm{E}+11$ & $8.232 \mathrm{E}+03$ & $6.186 \mathrm{E}+06$ \\
\hline
\end{tabular}




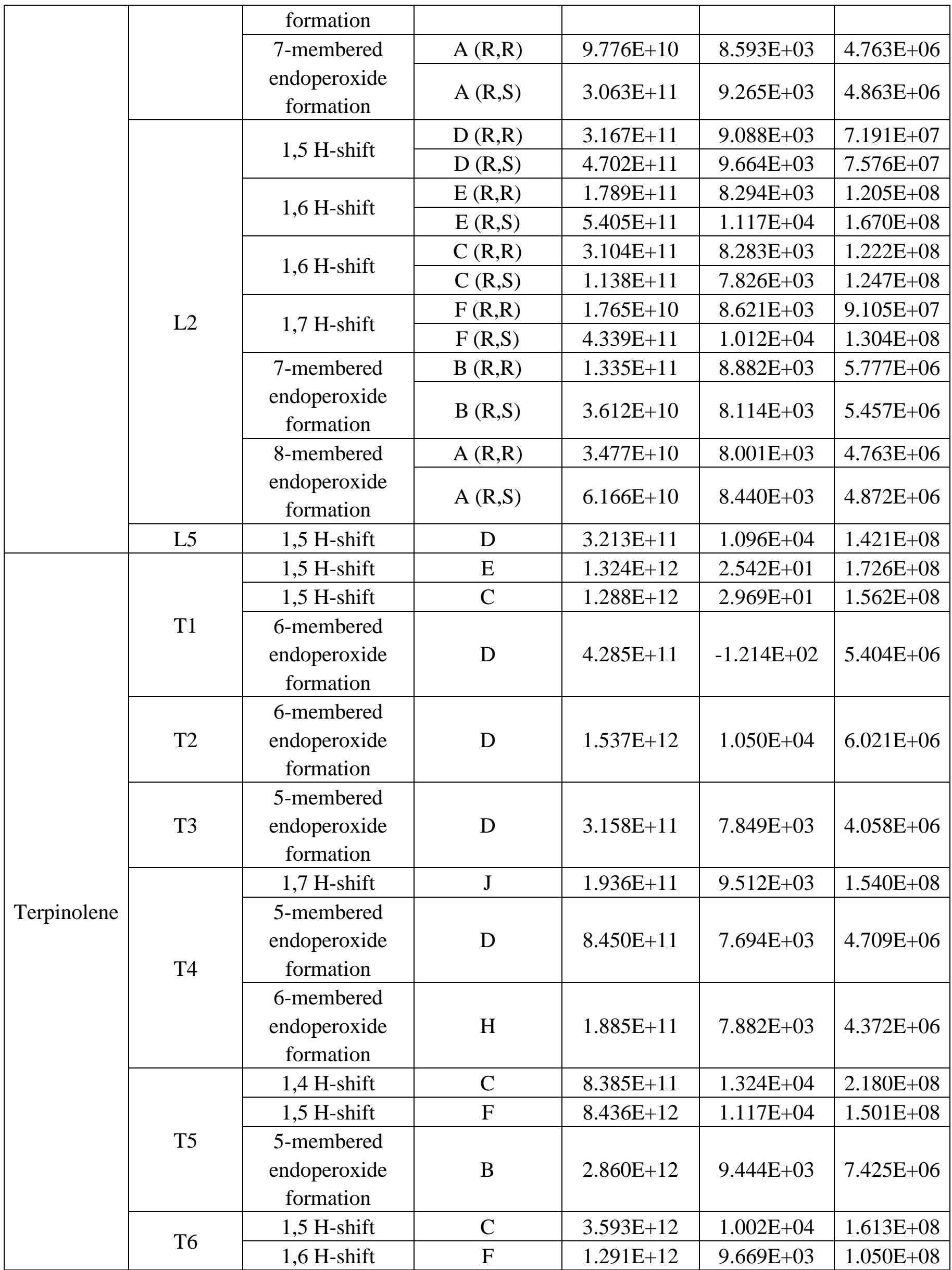




\begin{tabular}{|l|l|c|c|c|c|c|}
\hline & $\begin{array}{c}\text { 6-membered } \\
\text { endoperoxide } \\
\text { formation }\end{array}$ & B & $4.725 \mathrm{E}+11$ & $7.463 \mathrm{E}+03$ & $6.488 \mathrm{E}+06$ \\
\cline { 2 - 6 } & $\begin{array}{c}7 \text {-membered } \\
\text { endoperoxide } \\
\text { formation }\end{array}$ & A & $3.022 \mathrm{E}+11$ & $9.158 \mathrm{E}+03$ & $5.257 \mathrm{E}+06$ \\
\hline
\end{tabular}




\section{S6 Reverse Reactions}

The unimolecular reactions studied in this work are reversible. However, the reverse reactions compete with the addition of molecular oxygen, which occurs with typical pseudo-first order rate coefficients on the order of $10^{7}-10^{8} \mathrm{~s}^{-1}$. Thus, the reverse unimolecular reactions need to be faster than that to compete with $\mathrm{O}_{2}$-addition and thereby decrease the effective forward rate coefficient. For a set of representative, mainly fast, unimolecular reactions studied here (Table S 32 and Table S 33) we find that while the reverse reactions can be fast, they are slower than the competing $\mathrm{O}_{2}$-addition. The fastest reverse reactions are observed for the reactions with low forward reaction rate coefficients, as those typically lead to the least stable products. Thus, our calculations indicate that the reverse reactions are of little importance in the atmosphere.

Table S 32. Forward and reverse reaction barriers $\left(\Delta E_{f}\right.$ and $\Delta E_{r}$, respectively, in $\left.\mathrm{kcal} / \mathrm{mol}\right)$, MC-TST rate coefficients $\left(k_{f}\right.$ and $k_{r}$, respectively, in $\mathrm{s}^{-1}$ ) and reaction energy (defined as product - reactant, in $\mathrm{kcal} / \mathrm{mol}$ ) for an $\alpha$-pinene hydroxy peroxy radical. Rate coefficients are calculated using the approach by Møller et al. ${ }^{1}$

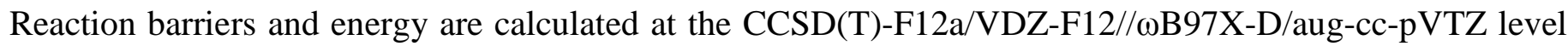
including $\omega$ B97X-D/aug-cc-pVTZ ZPVE. The reactions are defined according to Figure $\mathrm{S} 4$. All energies are based on lowest-energy conformers.

\begin{tabular}{|c|c|c|c|c|c|}
\hline Reaction & $\Delta E_{f}$ & $\Delta E_{r}$ & $k_{f}$ & $k_{r}$ & Reaction Energy \\
\hline A1-1,5E & 19.54 & 20.77 & 1.1 & 0.12 & 1.23 \\
\hline A1-1,5C & 25.45 & 11.03 & $6.0 \cdot 10^{-6}$ & $1.5 \cdot 10^{5}$ & -14.41 \\
\hline A1-1,6B & 18.37 & 22.09 & 0.37 & $7.5 \cdot 10^{-4}$ & 3.73 \\
\hline A1-endo-6F & 16.31 & 15.18 & 0.35 & 64 & -1.13 \\
\hline
\end{tabular}

Table S 33. Forward and reverse reaction barriers $\left(\Delta E_{f}\right.$ and $\Delta E_{r}$, respectively, in $\left.\mathrm{kcal} / \mathrm{mol}\right)$, MC-TST rate coefficients $\left(k_{f}\right.$ and $k_{r}$, respectively, in $\left.\mathrm{s}^{-1}\right)$ and reaction energy (defined as product - reactant, in $\mathrm{kcal} / \mathrm{mol}$ ) for limonene hydroxy peroxy radicals. Rate coefficients are calculated using the approach by Møller et al. ${ }^{1}$

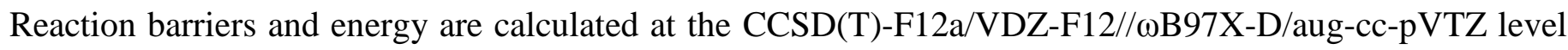
including $\omega$ B97X-D/aug-cc-pVTZ ZPVE. The reactions are defined according to Figure S 12. All energies are based on lowest-energy conformers.

\begin{tabular}{|c|c|c|c|c|c|}
\hline Reaction & $\Delta E_{f}$ & $\Delta E_{r}$ & $k_{f}$ & $k_{r}$ & Reaction Energy \\
\hline L1-endo-6B $(R, R)$ & 15.03 & 14.15 & 3.9 & $4.1 \cdot 10^{2}$ & -0.88 \\
\hline L1-endo-7A $(R, R)$ & 17.81 & 13.87 & $3.6 \cdot 10^{-2}$ & $1.0 \cdot 10^{3}$ & -3.94 \\
\hline L2-1,6C $(R, R)$ & 16.93 & 19.80 & 30 & 0.19 & 2.87 \\
\hline L2-endo-7B $(R, R)$ & 18.15 & 14.65 & $2.1 \cdot 10^{-2}$ & $2.9 \cdot 10^{2}$ & -3.50 \\
\hline L2-endo-8A $(R, R)$ & 16.27 & 13.87 & 0.17 & $1.0 \cdot 10^{3}$ & -2.40 \\
\hline
\end{tabular}


Table S 34. Forward and reverse reaction barriers $\left(\Delta E_{f}\right.$ and $\Delta E_{r}$, respectively, in $\left.\mathrm{kcal} / \mathrm{mol}\right)$, MC-TST rate coefficients $\left(k_{f}\right.$ and $k_{r}$, respectively, in $\mathrm{s}^{-1}$ ) and reaction energy (defined as product - reactant, in $\mathrm{kcal} / \mathrm{mol}$ ) for limonene hydroxy peroxy radicals. All values are calculated at the $\omega$ B97X-D/aug-cc-pVTZ level and all energies include ZPVE. The reactions are defined according to Figure S 12. All energies are based on lowest-energy conformers.

\begin{tabular}{|c|c|c|c|c|c|}
\hline Reaction & $\Delta E_{f}$ & $\Delta E_{r}$ & $k_{f}$ & $k_{r}$ & Reaction Energy \\
\hline L2-1,5D $(R, R)$ & 19.23 & 7.77 & $6.6 \cdot 10^{-2}$ & $1.1 \cdot 10^{7}$ & -11.46 \\
\hline L2-1,6C $(R, R)$ & 16.35 & 17.22 & 63 & 12 & 0.87 \\
\hline L2-endo-7B $(R, R)$ & 18.12 & 12.84 & $2.1 \cdot 10^{-2}$ & $6.3 \cdot 10^{3}$ & -5.28 \\
\hline L2-endo-8A $(R, R)$ & 18.46 & 1.89 & $4.2 \cdot 10^{-3}$ & $2.8 \cdot 10^{4}$ & -6.56 \\
\hline
\end{tabular}

Due to the relatively clear results from these test systems indicating that the reverse reactions are not generally competitive with addition of molecular oxygen, the reverse MC-TST rate coefficients have not been calculated for the remaining reactions. Instead, we have assessed the reaction energies (energy difference between product and reactant) based on the optimized IRC end-points (Table S 35-Table S 38). Positive values thus represent exothermic reactions and negative values represent endothermic reactions. While the reaction barriers (energy difference between reactant and TS or product and TS) are typically underestimated by the IRC barriers, the reaction energies are likely reasonable within a few $\mathrm{kcal} / \mathrm{mol}$, as can be seen by comparing Table S 32 and Table S 35 and Table S 33-Table S 34 and Table S 37 for $\alpha$-pinene and limonene, respectively. From Table S 35-Table S 38, it is clear that the unimolecular reactions with the fastest forward reactions are largely thermoneutral (reaction energies of $0 \mathrm{kcal} / \mathrm{mol}$ ). As such, the reverse reaction rate coefficients are expected to be comparable to the forward reaction rate coefficients, which are clearly uncompetitive with $\mathrm{O}_{2}$ addition $\left(k_{O_{2} \text {-addition }} \sim 10^{7}-10^{8} \mathrm{~s}^{-1}\right)$. This is true for both the allylic H-shifts and the endoperoxide cyclization reactions, which are the dominant unimolecular reactions. The reactions leading to alkyl radicals are generally endothermic by approximately $10 \mathrm{kcal} / \mathrm{mol}$ and will thus have much faster reverse reactions compared to the forward reactions. However, as the forward reactions are typically quite slow for these reactions, the reverse reactions are generally expected to remain uncompetitive with $\mathrm{O}_{2}$-addition.

Table S 35. Forward MC-TST rate coefficients $\left(k_{f}\right.$, in $\left.\mathrm{s}^{-1}\right)$ and reaction energies based on the energies of the optimized IRC end-points (in $\mathrm{kcal} / \mathrm{mol}$ ) calculated as (product - reactant) for $\alpha$-pinene hydroxy peroxy radicals. All values are calculated at the $\operatorname{CCSD}(\mathrm{T})-\mathrm{F} 12 \mathrm{a} / \mathrm{VDZ}-\mathrm{F} 12$ level. The reaction energy includes $\omega B 97 X-D /$ aug-cc-pVTZ ZPVE. The reactions are defined according to Figure S 4.

\begin{tabular}{|l|l|l|}
\hline Reaction & $k_{f}$ & IRC reaction energy \\
\hline A1-1,5E & 1.1 & 0.95 \\
\hline A1-1,5C & $6.0 \cdot 10^{-6}$ & -14.36 \\
\hline A1-1,6B & 0.37 & 6.54 \\
\hline A1-endo-6F & 0.35 & 3.38 \\
\hline A1-endo-7A & $2.0 \cdot 10^{-3}$ & 0.11 \\
\hline A10-1,5E & 0.16 & 4.40 \\
\hline A10-1,5C & $8.8 \cdot 10^{-6}$ & -15.89 \\
\hline A10-endo-6F & 2.3 & 3.37 \\
\hline A10-endo-7A & $2.6 \cdot 10^{-2}$ & 0.97 \\
\hline
\end{tabular}


Table S 36. Forward MC-TST rate coefficients $\left(k_{f}\right.$, in $\left.\mathrm{s}^{-1}\right)$ and reaction energies based on the energies of the optimized IRC end-points (in $\mathrm{kcal} / \mathrm{mol}$ ) calculated as (product - reactant) for $\beta$-pinene hydroxy peroxy radicals. All values are calculated at the $\operatorname{CCSD}(\mathrm{T})-\mathrm{F} 12 \mathrm{a} / \mathrm{VDZ}-\mathrm{F} 12$ level. The reaction energy includes $\omega B 97 X-D /$ aug-cc-pVTZ ZPVE. The reactions are defined according to Figure S 6.

\begin{tabular}{|l|l|l|}
\hline Reaction & $k_{f}$ & IRC reaction energy \\
\hline B2-1,5B & $8.1 \cdot 10^{-2}$ & -9.19 \\
\hline B5-1,5E & 1.4 & 0.75 \\
\hline B5-1,5C & $7.3 \cdot 10^{-6}$ & -12.43 \\
\hline B5-1,6B & 0.28 & 1.04 \\
\hline B5-endo-6F & 4.0 & 2.47 \\
\hline B5-endo-7A & $4.8 \cdot 10^{-2}$ & 0.45 \\
\hline
\end{tabular}

Table S 37. Forward MC-TST rate coefficients $\left(k_{f}\right.$, in s $\left.^{-1}\right)$ and reaction energies based on the energies of the optimized IRC end-points (in $\mathrm{kcal} / \mathrm{mol}$ ) calculated as (product - reactant) for limonene hydroxy peroxy radicals. All values are calculated at the $\operatorname{CCSD}(\mathrm{T})-\mathrm{F} 12 \mathrm{a} / \mathrm{VDZ}-\mathrm{F} 12$ level. The reaction energy includes $\omega B 97 X-D /$ aug-cc-pVTZ ZPVE. The reactions are defined according to Figure S 12.

\begin{tabular}{|l|l|l|}
\hline Reaction & $k_{f}$ & IRC reaction energy \\
\hline L1-1,5C $(R, R)$ & 4.0 & 1.49 \\
\hline L1-1,6F $(R, R)$ & 0.20 & 1.57 \\
\hline L1-endo-6B $(R, R)$ & 3.9 & 3.25 \\
\hline L1-endo-7A $(R, R)$ & $3.6 \cdot 10^{-2}$ & 0.19 \\
\hline L1-1,5C $(R, S)$ & 0.53 & 1.32 \\
\hline L1-1,6F $(R, S)$ & 0.69 & 0.60 \\
\hline L1-endo-6B $(R, S)$ & 0.93 & 2.86 \\
\hline L1-endo-7A $(R, S)$ & $1.2 \cdot 10^{-2}$ & 0.58 \\
\hline L2-1,5D $(R, R)$ & 0.31 & -9.43 \\
\hline L2-1,6E $(R, R)$ & $7.0 \cdot 10^{-3}$ & -11.09 \\
\hline L2-1,6C $(R, R)$ & 30 & 3.50 \\
\hline L2-1,7F $(R, R)$ & 0.17 & 0.32 \\
\hline L2-endo-7B $(R, R)$ & $2.1 \cdot 10^{-2}$ & -0.31 \\
\hline L2-endo-8A $(R, R)$ & 0.17 & 0.19 \\
\hline L2-1,5D $(R, S)$ & $6.9 \cdot 10^{-2}$ & -9.90 \\
\hline L2-1,6E $(R, S)$ & $1.2 \cdot 10^{-3}$ & -11.33 \\
\hline L2-1,6C $(R, S)$ & 36 & 4.16 \\
\hline L2-1,7F $(R, S)$ & $8.1 \cdot 10^{-2}$ & -0.26 \\
\hline L2-endo-7B $(R, S)$ & $6.7 \cdot 10^{-2}$ & 0.40 \\
\hline L2-endo-8A $(R, S)$ & $3.8 \cdot 10^{-2}$ & 0.58 \\
\hline L5-1,5D & $7.8 \cdot 10^{-3}$ & -1.12 \\
\hline
\end{tabular}


Table S 38. Forward MC-TST rate coefficients $\left(k_{f}\right.$, in $\left.\mathrm{s}^{-1}\right)$ and reaction energies based on the energies of the optimized IRC end-points (in kcal/mol) calculated as (product - reactant) for terpinolene hydroxy peroxy radicals. All values are calculated at the CCSD(T)-F12a/VDZ-F12 level. The reaction energy includes $\omega B 97 X-D /$ aug-cc-pVTZ ZPVE. The reactions are defined according to Figure S 14.

\begin{tabular}{|l|l|l|}
\hline Reaction & $k_{f}$ & IRC reaction energy \\
\hline T1-1,5E & $1.2 \cdot 10^{-2}$ & -1.48 \\
\hline T1-1,5C & $1.0 \cdot 10^{-2}$ & -3.52 \\
\hline T1-endo-6D & $5.4 \cdot 10^{-2}$ & 4.63 \\
\hline T2-endo-6D & $9.8 \cdot 10^{-4}$ & 1.90 \\
\hline T3-endo-5D & 1.4 & 4.70 \\
\hline T4-1,7J & 0.92 & -0.27 \\
\hline T4-endo-5D & 6.3 & 4.14 \\
\hline T4-endo-6H & 0.73 & 0.83 \\
\hline T5-1,4C & $5.5 \cdot 10^{-3}$ & 1.75 \\
\hline T5-1,5F & 0.13 & -2.96 \\
\hline T5-endo-5B & $6.6 \cdot 10^{-2}$ & -1.50 \\
\hline T6-1,5C & 4.1 & 0.17 \\
\hline T6-1,6F & 0.57 & 0.91 \\
\hline T6-endo-6B & 8.1 & 3.62 \\
\hline T6-endo-7A & $1.7 \cdot 10^{-2}$ & 0.36 \\
\hline
\end{tabular}

As opposed to the reactions in the tables above, the $\mathrm{OH} \mathrm{H}$-shifts ( $\mathrm{H}$-shifts abstracting hydrogens from $\mathrm{OH}-$ groups) are highly endothermic (by approximately $20 \mathrm{kcal} / \mathrm{mol}$ ) and have very low reverse barriers (Table $\mathrm{S}$ 39). Thus, the $\mathrm{OH} \mathrm{H}$-shifts are likely to revert to the reactants should they occur. However, alkoxy radicals are highly reactive and may have other unimolecular reactions, e.g. bond scissions, with very large rate coefficients and their dominant reaction pathways thus cannot accurately be predicted without a more detailed study.

Table S 39. Forward and reverse IRC barriers $\left(\Delta E_{f, I R C}\right.$ and $\Delta E_{r, I R C}$, respectively, in $\left.\mathrm{kcal} / \mathrm{mol}\right)$ of the $\mathrm{OH} \mathrm{H}-$ shifts in the different peroxy radicals studied. All values are calculated at the $\omega B$ 97X-D/aug-cc-pVTZ level and include ZPVE.

\begin{tabular}{|c|c|c|}
\hline Peroxy Radical & $\Delta E_{f, I R C}$ & $\Delta E_{r, I R C}$ \\
\hline A2 & 18.18 & 0.09 \\
\hline A4 & 18.10 & -0.30 \\
\hline A7 & 17.90 & 0.92 \\
\hline A9 & 17.72 & 0.47 \\
\hline A10 & 24.30 & 3.28 \\
\hline B1 & 20.74 & 0.66 \\
\hline B2 & 19.09 & 1.43 \\
\hline B3 & 21.10 & -0.35 \\
\hline B4 & 19.92 & -0.35 \\
\hline D1 & 21.53 & 0.50 \\
\hline
\end{tabular}




\begin{tabular}{|c|c|c|}
\hline D2 & 21.28 & 0.70 \\
\hline D3 & 21.30 & 0.58 \\
\hline D4 & 23.11 & 1.03 \\
\hline D5 & 22.97 & 1.06 \\
\hline D6 & 19.91 & 0.03 \\
\hline D7 & 19.65 & 0.30 \\
\hline D8 & 18.96 & 0.31 \\
\hline $\mathrm{C} 1$ & 20.55 & -0.41 \\
\hline $\mathrm{C} 2$ & 18.66 & -0.69 \\
\hline C3 & 19.59 & 0.49 \\
\hline $\mathrm{C} 4$ & 18.57 & 0.39 \\
\hline L1 $(\mathrm{R}, \mathrm{R})$ & 21.34 & -0.28 \\
\hline $\mathrm{L} 1(\mathrm{R}, \mathrm{S})$ & 22.12 & 0.14 \\
\hline $\mathrm{L} 2(\mathrm{R}, \mathrm{R})$ & 20.22 & 0.39 \\
\hline L2 $(\mathrm{R}, \mathrm{S})$ & 19.96 & 0.25 \\
\hline L3 & 19.80 & 0.10 \\
\hline $\mathrm{L} 4$ & 22.42 & 0.74 \\
\hline L5 & 19.10 & 0.03 \\
\hline L6 & 20.96 & 0.65 \\
\hline L7 & 22.52 & 1.08 \\
\hline L8 & 20.43 & 0.24 \\
\hline L9 & 20.65 & 0.66 \\
\hline L10 & 21.05 & 0.49 \\
\hline $\mathrm{T} 1$ & 20.09 & 0.18 \\
\hline $\mathrm{T} 2$ & 22.36 & 1.04 \\
\hline $\mathrm{T} 3$ & 20.95 & 0.40 \\
\hline $\mathrm{T} 4$ & 21.08 & 0.71 \\
\hline T5 & 20.30 & -0.46 \\
\hline T6 & 19.82 & -0.48 \\
\hline
\end{tabular}




\section{References}

1. Møller, K. H.; Otkjær, R. V.; Hyttinen, N.; Kurtén, T.; Kjaergaard, H. G., Cost-Effective Implementation of Multiconformer Transition State Theory for Peroxy Radical Hydrogen Shift Reactions. J. Phys. Chem. A 2016, 120 (51), 10072-10087.

2. Xu, L.; Møller, K. H.; Crounse, J. D.; Otkjær, R. V.; Kjaergaard, H. G.; Wennberg, P. O., Unimolecular Reactions of Peroxy Radicals Formed in the Oxidation of $\alpha$-Pinene and $\beta$-Pinene by Hydroxyl Radicals. $J$. Phys. Chem. A 2019, 123 (8), 1661-1674.

3. Berndt, T.; Richters, S.; Jokinen, T.; Hyttinen, N.; Kurtén, T.; Otkjær, R. V.; Kjaergaard, H. G.; Stratmann, F.; Herrmann, H.; Sipilä, M.; Kulmala, M.; Ehn, M., Hydroxyl Radical-Induced Formation of Highly Oxidized Organic Compounds. Nature Comm. 2016, 7 (1), 13677.

4. Gaona-Colmán, E.; Blanco, M. B.; Barnes, I.; Wiesen, P.; Teruel, M. A., OH- and O3-Initiated Atmospheric Degradation of Camphene: Temperature Dependent Rate Coefficients, Product Yields and Mechanisms. RSC Adv. 2017, 7 (5), 2733-2744.

5. $\mathrm{Wu}, \mathrm{D}$.; Bayes, K. D., Rate Constants for the Reactions of Isobutyl, Neopentyl, Cyclopentyl, and Cyclohexyl Radicals with Molecular Oxygen. Int. J. Chem. Kin. 1986, 18 (5), 547-554.

6. Forst, W., Analytic Solution of Relaxation in a System with Exponential Transition Probabilities. III. Macroscopic Disequilibrium. J. Chem. Phys. 1984, 80 (6), 2504-2513.

7. Kurtén, T.; Rissanen, M. P.; Mackeprang, K.; Thornton, J. A.; Hyttinen, N.; Jørgensen, S.; Ehn, M.; Kjaergaard, H. G., Computational Study of Hydrogen Shifts and Ring-Opening Mechanisms in $\alpha$-Pinene Ozonolysis Products. J. Phys. Chem. A 2015, 119 (46), 11366-11375.

8. Cuadros, F.; Mulero, A.; Cachadiña, J.; Ahumada, W., A New Procedure for Determining Lennard-Jones Interaction Parameters. Int. Rev. Phys. Chem. 1995, 14 (2), 205-213.

9. Peeters, J.; Müller, J.-F.; Stavrakou, T.; Nguyen, V. S., Hydroxyl Radical Recycling in Isoprene Oxidation Driven by Hydrogen Bonding and Hydrogen Tunneling: The Upgraded LIM1 Mechanism. J. Phys. Chem. A 2014, 118 (38), 8625-8643.

10. Praske, E.; Otkjær, R. V.; Crounse, J. D.; Hethcox, J. C.; Stoltz, B. M.; Kjaergaard, H. G.; Wennberg, P. O., Atmospheric Autoxidation is Increasingly Important in Urban and Suburban North America. Proc. Natl. Acad. Sci. 2018, 115 (1), 64. 